\title{
ON A QUESTION OF DRINFELD ON THE WEIL REPRESENTATION: THE FINITE FIELD CASE
}

\author{
CHUN-HUI WANG
}

\begin{abstract}
Let $F$ be a finite field of odd cardinality, and let $G=\mathrm{GL}_{2}(F)$. The group $G \times G \times G$ acts on $F^{2} \otimes F^{2} \otimes F^{2}$ via symplectic similitudes, and has a natural Weil representation. Answering a question raised by V. Drinfeld, we decompose that representation into irreducibles. We also decompose the analogous representation of $\mathrm{GL}_{2}(A)$, where $A$ is a cubic algebra over $F$.
\end{abstract}

\section{INTRODUCTION}

Let $F$ be a finite field of odd cardinality $q$, and let $W,\langle$,$\rangle be a symplectic vector space over F$ of dimension $2 n$. The Heisenberg group $\mathrm{H}(W)$, attached to $W$ and $F$, is a set $W \oplus F$ with the group law: $(w, t)\left(w^{\prime}, t^{\prime}\right)=\left(w+w^{\prime}, t+t^{\prime}+\frac{\left\langle w, w^{\prime}\right\rangle}{2}\right)$. Let $\operatorname{Sp}(W)$ be the isometry group of $(W,\langle\rangle$,$) . Define a semi-direct product$ group $\mathrm{H}(W) \rtimes \mathrm{Sp}(W)$ by $[(w, t), g] \cdot\left[\left(w^{\prime}, t^{\prime}\right), g^{\prime}\right]=\left[(w, t)+\left(g \cdot w^{\prime}, t^{\prime}\right), g g^{\prime}\right]$. Fix a non-trivial character $\psi$ of $F$. According to the Stone-Von Neumann theorem, there is only one equivalence class of irreducible complex representation $\omega_{\psi}$ of $\mathrm{H}(W)$ with central character $\psi$. By Weil's celebrated paper [14], in fact $\omega_{\psi}$ is a representation of $\mathrm{H}(W) \rtimes \mathrm{Sp}(W)$ in the finite field case. The restriction of $\omega_{\psi}$ to $\operatorname{Sp}(W)$, now is well-known as the Weil representation; in [6], Gérardin investigated fully this representation. Following Shinoda [12], we extend it to the symplectic similitude group $\operatorname{GSp}(W)$ by setting $\rho=\operatorname{Ind}_{\mathrm{Sp}(W)}^{\mathrm{GSp}(W)} \omega_{\psi}$, which does not depend on the choice of $\psi$ ([12, p. 270, Theorem]).

The initial question raised by V.Drinfeld, in the finite field case, is understood roughly in the following way. Let $F^{2},\langle$,$\rangle be a symplectic space over F$ of dimension 2. Consider now $W=F^{2} \otimes F^{2} \otimes F^{2}$, a symplectic vector space over $F$ of dimension 8 endowed with the symplectic form $\langle,\rangle_{F^{2}} \otimes\langle,\rangle_{F^{2}} \otimes\langle,\rangle_{F^{2}}$. So there is a map from $\mathrm{GL}_{2}(F) \times \mathrm{GL}_{2}(F) \times \mathrm{GL}_{2}(F)$ to $\mathrm{GSp}(W)$. In this way we can define a Weil representation $\pi$ for $\mathrm{GL}_{2}(F) \times \mathrm{GL}_{2}(F) \times \mathrm{GL}_{2}(F)$ via the restriction of $\rho$, where $\rho$ is the Weil representation of $\mathrm{GSp}(W)$. The question is asked about the set of the quotients of $\pi$. Does it contain the representations of the form $\sigma \otimes \sigma \otimes \sigma$ for any irreducible representation $\sigma$ of $\mathrm{GL}_{2}(F)$ ? In this paper, we answer this question and also consider its variant version. To be precise, suppose now that $E / F$ (resp. $K / F$ ) is a field extension of degree 2 (resp. 3). Take $A$ to be an étale algebra over $F$ of degree 3, so $A$ is isomorphic to one of the algebras $F \times F \times F, F \times E, K$. We shall construct a homomorphism from $\mathrm{GL}_{2}(A)$ to $\mathrm{GSp}_{8}(F)$. If $A=F \times F \times F$, then $\mathrm{GL}_{2}(A) \simeq \mathrm{GL}_{2}(F) \times \mathrm{GL}_{2}(F) \times \mathrm{GL}_{2}(F)$. This goes back to Drinfeld's question. If $A=F \times E$, then $\mathrm{GL}_{2}(A) \simeq \mathrm{GL}_{2}(F) \times \mathrm{GL}_{2}(E)$. By Weil's Galois descent, we construct a quadratic vector space $M=\left\{\left(\begin{array}{ll}x & \alpha \\ \alpha & y\end{array}\right) \mid x, y \in F, \alpha \in E\right\}$ over $F$ of dimension 4, with the quadratic form $Q$ defined by the determinant of the matrix. Clearly there is a map from $\mathrm{GL}_{2}(E)$ to $\mathrm{GO}(Q)$, which is defined by $h \cdot m=h m \bar{h}^{t}$, where $h \in \mathrm{GL}_{2}(E), m \in M$ and $\bar{h}^{t}$ is the conjugate transpose of $h$. So $F^{2} \otimes M$ is a symplectic vector space over $F$ of dimension 8 , and there is a map from $\mathrm{GL}_{2}(F) \times \mathrm{GL}_{2}(E)$ to $\mathrm{GSp}_{8}(F)$. If $A=K$, in this situation, we also need to use Weil's Galois descent to construct a map from $\mathrm{GL}_{2}(K)$ to $\mathrm{GSp}_{8}(F)$. The map from $\mathrm{GL}_{2}(A)$ to $\mathrm{GSp}_{8}(F)$ leads us to define a representation $\pi_{A}$ of $\mathrm{GL}_{2}(A)$ via the restriction of $\rho$. The main purpose of this paper is to obtain the complete decomposition of $\pi_{A}$ in each case.

Date: September 2013

2000 Mathematics Subject Classification. 11F27, 20C33 (Primary).

Key words and phrases. Weil representation, base change, Shintani lift. 
For the group $G=\mathrm{GL}_{2}(F)$, we write $1_{G}$ for the trivial representation of $G$, and $\mathrm{St}_{G}$ for the Steinberg representation of $G$. Let $T=\left\{\left(\begin{array}{ll}a & 0 \\ 0 & d\end{array}\right) \in G\right\}, B=\left\{\left(\begin{array}{ll}a & b \\ 0 & d\end{array}\right) \in G\right\}$. Let $\chi_{1} \otimes \chi_{2}$ be the character of $T$ defined by $\left(\begin{array}{ll}a & 0 \\ 0 & d\end{array}\right) \longmapsto \chi_{1}(a) \chi_{2}(d)$ for two characters $\chi_{1}, \chi_{2}$ of $F^{\times}$. We will denote the principal series representation $\operatorname{Ind}_{B}^{G}\left(\chi_{1} \otimes \chi_{2}\right)$ of $G$ by $\pi_{\chi_{1}, \chi_{2}}$. If $(\sigma, V)$ is a representation of $G$ and $\psi$ a character of $F^{\times}$, we write the $\psi \cdot \sigma$ for the representation $\psi \cdot \sigma(g) \stackrel{=}{=}(\operatorname{det} g) \sigma(g)$. Let $\operatorname{Irr}(G)$ denote the class of all irreducible complex representations of the group $G$. Let $L$ be a field extension of $F$. By Shintani's work [13], one knows that there exists the base-change map $\mathrm{Bc}_{L / F}: \operatorname{Irr}\left(\mathrm{GL}_{2}(F)\right) \longrightarrow \operatorname{Irr}\left(\mathrm{GL}_{2}(L)\right)$, which is determined by character equalities. Our main results may be formulated as follows:

Theorem (1). If $A=F \times F \times F, \mathrm{GL}_{2}(A) \simeq \mathrm{GL}_{2}(F) \times \mathrm{GL}_{2}(F) \times \mathrm{GL}_{2}(F)$, then

$\pi_{A} \simeq \bigoplus_{\sigma \in \operatorname{Irr}\left(\mathrm{GL}_{2}(F)\right)} \sigma \otimes \sigma \otimes \sigma \oplus \bigoplus_{\psi \in \operatorname{Irr}\left(F^{\times}\right)}\left(\left(\psi \mathrm{St}_{\mathrm{GL}_{2}(F)} \otimes \psi \cdot 1_{\mathrm{GL}_{2}(F)} \otimes \psi \cdot 1_{\mathrm{GL}_{2}(F)}\right) \oplus\left(\psi \cdot 1_{\mathrm{GL}_{2}(F)} \otimes\left\langle\mathrm{St}_{\mathrm{GL}_{2}(F)} \otimes \psi \cdot 1_{\mathrm{GL}_{2}(F)}\right) \oplus\left(\psi \cdot 1_{\mathrm{GL}_{2}(F)} \otimes \psi \cdot 1_{\mathrm{GL}_{2}(F)} \otimes / \mathrm{St}_{\mathrm{GL}_{2}(F)}\right)\right)\right.$

Theorem (2). If $A=F \times E, \mathrm{GL}_{2}(A) \simeq \mathrm{GL}_{2}(F) \times \mathrm{GL}_{2}(E)$, then

$$
\pi_{A} \simeq \bigoplus_{\sigma \in \operatorname{Irr}\left(\mathrm{GL}_{2}(F)\right)} \sigma \otimes \mathrm{Bc}_{E / F}(\sigma) \oplus \bigoplus_{\psi \in \operatorname{Irr}\left(F^{\times}\right), \Psi \in \operatorname{Irr}\left(E^{\times}\right), \Psi=\psi \circ \mathrm{N}_{E / F}}\left(\psi \mathrm{St}_{\mathrm{GL}_{2}(F)} \otimes \Psi \cdot 1_{\mathrm{GL}_{2}(E)}\right)
$$

Theorem (3). If $A=K, \mathrm{GL}_{2}(A) \simeq \mathrm{GL}_{2}(K)$, then

$$
\pi_{A} \simeq \bigoplus_{\sigma \in \operatorname{Irr}\left(\mathrm{GL}_{2}(F)\right)} \mathrm{Bc}_{K / F}(\sigma)
$$

Let us briefly review the whole story. Theorems (1) is obtained mainly by using the method in [1] to decompose reducible representations. In [1], Andrade considered higher rank groups. We first formulate the representation $\pi_{A}$ of $\mathrm{GL}_{2}(F) \times \mathrm{GL}_{2}(F) \times \mathrm{GL}_{2}(F)$ concerned in this case. This can be done by following works of Gérardin and of Shinoda on the Weil representations(cf. [6], [12]). Then we take two irreducible representations $\pi_{1}, \pi_{2}$ of $\mathrm{GL}_{2}(F)$, and determine the dimension of $\operatorname{Hom}_{1 \times \mathrm{GL}_{2}(F) \times \mathrm{GL}_{2}(F)}\left(\pi_{A}, \pi_{1} \otimes \pi_{2}\right)$. One key ingredient is that $\pi_{A}$ is in fact a representation of the group $\left(\mathrm{GL}_{2}(F) \times \mathrm{GL}_{2}(F) \times \mathrm{GL}_{2}(F)\right) \rtimes S_{3}$, where $S_{3}$ is the permutation group of 3 variables. So if we put $\mathcal{R}\left(\pi_{A}\right)=\left\{\pi_{1} \otimes \pi_{2} \otimes \pi_{3} \mid \operatorname{Hom}_{\mathrm{GL}_{2}(F) \times \mathrm{GL}_{2}(F) \times \mathrm{GL}_{2}(F)}\left(\pi_{A}, \pi_{1} \otimes \pi_{2} \otimes \pi_{3}\right) \neq 0\right\}$, by Clifford theory, $\mathcal{R}\left(\pi_{A}\right)$ is $S_{3}$-invariant. This together with the above calculations of dimension derives Theorem (1). For Theorem (2), following the method in [1], we first write down the Weil representation $\pi_{A}$ of $\mathrm{GL}_{2}(F) \times \mathrm{GL}_{2}(E)$ in this case, and then decompose the canonical representation $\left(\operatorname{GL}_{2}(F), \operatorname{Hom}_{\mathrm{GL}_{2}(E)}\left(\pi_{A}, \Pi\right)\right)$ into irreducibles for each $\Pi \in \operatorname{Irr}\left(\mathrm{GL}_{2}(E)\right)$. We did this by checking the irreducible representations of $\mathrm{GL}_{2}(E)$ one by one. The main difficulty is when $\Pi$ is cuspidal. For that case, we use the explicit models given by [1]. The étale algebra $A=K$ is a new case. We use Weil's Galois descent to construct a map $i$ from $\mathrm{GL}_{2}(K)$ to $\operatorname{GSp}_{8}(F)$. Through this map, we shall define a new Weil representation $\pi_{A}$ for the group $\mathrm{GL}_{2}(K)$. However the explicit realisation of this representation is somehow complex, this causes the difficulty to study its irreducible components. One point is that the map $i$ sends the standard Borel subgroup of $\mathrm{GL}_{2}(K)$ to that of $\mathrm{GSp}_{8}(F)$. By virtue of Frobenius reciprocity, we obtain the results for the principal series representations. For the cuspidal representations, we use a technique so-called "base change" to reduce to deal with some principal series representations. We should mention that this technique has been used in Gan's paper [5] to obtain Howe correspondences for exceptional groups.

Another approach to the results of this paper maybe use character theory in representations and it sometimes involves to solve certain equations. In practice, giving such equations in some sense is also complex.

The structure of this paper is as follows. The first section is devoted to giving some notations and recalling some known results. In the second section, we consider the étale algebra $A=F \times F \times F$. In the third section, we deal with the case $A=F \times E$. In the fourth section, we consider the case $A=K$; there we put some calculations in two appendices.

Acknowledgements. The paper is one part of the author's Ph.D. thesis and completes in the Academy of Mathematics and Systems Science. He would like to thank his advisor Guy Henniart for useful comments. 


\section{Notation and Preliminaries}

1.1. The following notations will be standard through the whole paragraph, and used repeatedly without recalling their meanings:

- $F=$ a finite field with odd cardinality $q$;

- $E$ = a fixed field extension of $F$ of degree 2;

- $K=$ a fixed field extension of $F$ of degree 3;

- $\phi=$ a fixed non-trivial character of the additive group $F$;

- $\phi^{a}=$ the character of $F$, defined by $\phi^{a}(b):=\phi(a b)$ for $b \in F, a \in F^{\times}$;

- $X_{A}=$ the set of all non-trivial irreducible complex representations of an abelian group $A$;

- $\operatorname{Rep}(G)=$ the category of complex representations of a finite group $G$;

- $\operatorname{Irr}(G)=$ the class of all irreducible complex representations of a finite group $G$, up to isomorphism;

- $\check{\sigma}=$ the contragredient representation of $\sigma$, for $\sigma \in \operatorname{Rep}(G)$;

- If $(\sigma, V)$ is a representation of $G$, then we will denote its $G$-invariant set by $V^{G}$.

1.2. For later use, we regroup some results of the Weil representation of $\operatorname{GSp}_{2 n}(F)$ (cf. [6], [10], [12]).

Let $V$ be a 2 n-dimensional $F$-vector space, endowed with a non-degenerate symplectic form \langle\rangle . To each nontrivial character $\psi$ of the additive group $F$, one can associate the Weil representation $\left(\omega_{\psi}, W_{\psi}\right)$ of the metaplectic group $\operatorname{Mp}_{2 n}(F)$ (cf. [10, Chapter 2]). The exact sequence

$$
1 \longrightarrow \mathbb{C}^{\times} \longrightarrow \operatorname{Mp}_{2 n}(F) \stackrel{p}{\longrightarrow} \operatorname{Sp}_{2 n}(F) \longrightarrow 1
$$

is splitting. Except $n=1, F=\mathbb{F}_{3}$, the group $\operatorname{Sp}_{2 n}(F)$ is perfect, so there exists a unique section of morphism $i$

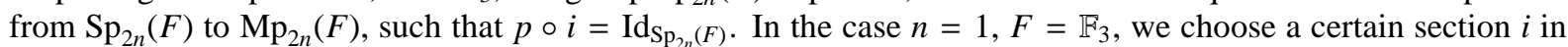
the sense of Gérardin (cf. [6, p. 63, Theorem 2.4 (a")]). Via the map $i$, one obtains a representation $\left(\omega_{\psi}, W_{\psi}\right)$ of $\mathrm{Sp}_{2 n}(F)$ with respect to $\psi$, called the Weil representation. One can extend it as a representation of GSp ${ }_{2 n}(F)$ by setting $\rho_{\psi}=\operatorname{Ind}_{\mathrm{Sp}_{2 n}(F)}^{\mathrm{GSp}_{2 n}(F)} \omega_{\psi}$. It is observed that $\rho_{\psi}$ is independent of $\psi$ (see [12, p. 270, Theorem]). Hence we could omit $\psi$, and only write $\rho$ briefly.

The study of the Weil representation often involves an explicit realized model. We recall one so-called “ the Schrödinger model": Let $V=V_{+} \oplus V_{-}$be a complete polarization. Let $\left\{v_{1}, \ldots, v_{n}\right\}$ be a F-basis of $V_{+}$, and $\left\{v_{1}^{\prime}, \ldots, v_{n}^{\prime}\right\}$ its dual basis with respect to $\langle$,$\rangle . Every element g \in \operatorname{GSp}(V)$ can be written in the following form: $g=\left(\begin{array}{cc}\alpha & \beta \\ \gamma & \delta\end{array}\right)$ where $\alpha \in \operatorname{End}_{F}\left(V_{+}\right), \beta \in \operatorname{Hom}_{F}\left(V_{-}, V_{+}\right), \gamma \in \operatorname{Hom}_{F}\left(V_{+}, V_{-}\right), \delta \in \operatorname{End}_{F}\left(V_{-}\right)$. The group $\operatorname{GSp}(V)$ is generated by the set $\left\{h(a), u(b), h^{\prime}(t), \omega\right\}$ (see [1], p. 163), where $h(a)=\left(\begin{array}{cc}a & 0 \\ 0 & a^{\vee}\end{array}\right), a^{\vee}$ is the contragredient of $a ; u(b)=\left(\begin{array}{ll}1 & b \\ 0 & 1\end{array}\right) \quad$ for a symmetric morphism $b \in \operatorname{Hom}_{F}\left(V_{-}, V_{+}\right) ; h^{\prime}(t)=\left(\begin{array}{cc}1 & 0 \\ 0 & t\end{array}\right), t \in F^{\times} ; \omega=\left(\begin{array}{cc}0 & I \\ -I & 0\end{array}\right)$ with $\omega\left(v_{i}\right)=-v_{i}^{\prime}, \omega\left(v_{i}^{\prime}\right)=v_{i}$ 1] The Weil representation $\rho$ of $\operatorname{GSp}(V)$ can be realized in the space $W_{-}=\mathbb{C}\left[V_{-} \times X_{F}\right]$ of complex functions on $V_{-} \times X_{F}$. More precisely the action of $\operatorname{GSp}(V)$ on $W_{-}$is determined by the following formulas (cf. [12, p. 270]):

$$
\begin{gathered}
(\rho(h(a)) F)(y, \psi)=\chi_{q}^{+}\left(\operatorname{det}_{V_{+}} a\right) F\left(a^{\vee-1} y, \psi\right), \\
(\rho(u(b)) F)(y, \psi)=\psi\left(\frac{1}{2}\langle b y, y\rangle\right) F(y, \psi), \\
(\rho(\omega) F)(y, \psi)=\gamma\left(\psi^{-\frac{1}{2}}\right)^{-n} \sum_{z \in V_{-}} F(z, \psi) \psi\left(\left\langle z, \omega^{-1}(y)\right\rangle\right), \\
\left(\rho\left(h^{\prime}(t)\right) F\right)(y, \psi)=F\left(y, \psi^{t^{-1}}\right),
\end{gathered}
$$

where $y \in V_{-}, \psi \in X_{F}, \gamma(\psi)=\sum_{x \in F} \psi\left(x^{2}\right), x_{q}^{+}=$Legendre symbol $\left(\overline{\mathbb{F}}_{q}\right)$.

\footnotetext{
${ }^{1}$ In [12], the $\omega$ is defined as $\omega\left(v_{i}\right)=v_{i}^{\prime}, \omega\left(v_{i}^{\prime}\right)=-v_{i}$, but it does not affect the following equation [1.3). To obtain [1.3], we use the equality: $\gamma\left(\psi^{\frac{1}{2}}\right)^{-n} \chi_{q}^{+}(-1)^{n}=\gamma\left(\psi^{-\frac{1}{2}}\right)^{-n}$.
} 
1.3. We summarize some facts about the irreducible representations of $\mathrm{GL}_{2}(F)$ and its Borel subgroup (cf. [2, Chapter 2] and [11]).

We write $G=\mathrm{GL}_{2}(F), B=\left\{\left(\begin{array}{ll}a & b \\ 0 & d\end{array}\right) \in G\right\}, N=\left\{\left(\begin{array}{ll}1 & b \\ 0 & 1\end{array}\right) \in G\right\}, T=\left\{\left(\begin{array}{ll}a & 0 \\ 0 & d\end{array}\right) \in G\right\}, M=\left\{\left(\begin{array}{ll}a & b \\ 0 & 1\end{array}\right) \in G\right\}, Z=$ $\left\{\left(\begin{array}{ll}a & 0 \\ 0 & a\end{array}\right) \in G\right\}$. Recall that $1_{G}$ is the trivial representation of $G$, and $\mathrm{St}_{G}$ is the Steinberg representation of $G$. Let $\theta$ be a regular character of $E^{\times}$; the irreducible cuspidal representation of $G$ corresponding to $\theta$ will be denoted by $\pi_{\theta}$. If $(\sigma, V)$ is a representation of $G$ and $\psi$ a character of $F^{\times}$, we define the representation $(\psi \cdot \sigma, V)$ of $G$ by $\psi \cdot \sigma(g)=\psi(\operatorname{det} g) \sigma(g)$.

Theorem 1.1 ([2, Chapter 2]). The following is a complete list of the isomorphism classes of the irreducible representations of $G$ :

(1) $\pi_{\chi_{1}, \chi_{2}}, \quad$ where $\chi_{1} \neq \chi_{2}$ are characters of $F^{\times}$;

(2) $\psi \cdot 1_{G}$, where $\psi$ ranges over the characters of $F^{\times}$;

(3) $\psi \cdot \mathrm{St}_{G}$, where $\psi$ ranges over the characters of $F^{\times}$;

(4) $\pi_{\theta}$, where $\theta$ ranges over the regular characters of $E^{\times}$.

The classes in the list all are distinct except that in (1) $\pi_{\chi_{1}, \chi_{2}} \simeq \pi_{\chi_{2}, \chi_{1}}$, and in (4) $\pi_{\theta} \simeq \pi_{\theta q}$.

Lemma 1.2 ([2, Chapter 2]). Notations being in above Theorem, we then have $\left(\pi_{\chi_{1}, \chi_{2}}\right)^{\vee} \simeq \pi_{\chi_{1}^{-1}, \chi_{2}^{-1}},\left(\psi \cdot 1_{G}\right)^{\vee} \simeq$ $\psi^{-1} \cdot 1_{G},\left(\psi \cdot \mathrm{St}_{G}\right)^{\vee} \simeq \psi^{-1} \cdot \mathrm{St}_{G}$ and $\left(\pi_{\theta}\right)^{\vee} \simeq \pi_{\theta^{-1}}$.

Now we investigate the representations of the group B. Let $\sigma_{\chi_{1}, \chi_{2}}$ be the character of $B$, defined by $\sigma_{\chi_{1}, \chi_{2}}\left(\left(\begin{array}{ll}a & b \\ 0 & d\end{array}\right)\right)=\chi_{1}(a) \chi_{2}(d)$. Let $\sigma$ be the unique irreducible representation of $M$ of the highest dimension and $\psi$ a character of $F^{\times}$. Attached to $\sigma$ and $\psi$, there is an irreducible representation $\psi \otimes \sigma$ of $B$, defined by $(\psi \otimes \sigma)(z m):=\psi(z) \sigma(m)$ for $z \in Z, m \in M$.

Theorem 1.3 ([11, Theorem 7.1]). The following is a complete list of the isomorphism classes of the irreducible representations of $B$ :

(1) $\sigma_{\chi_{1}, \chi_{2}}$ for any pair $\left(\chi_{1}, \chi_{2}\right)$ of characters of $F^{\times}$;

(2) $\psi \otimes \sigma$ for any character $\psi$ of $Z$. $G$.

For convenience use, we describe the decomposition of the restriction to $B$ of any irreducible representation of

Proposition 1.4. (1) $\operatorname{Res}_{B}^{G}\left(\psi \cdot 1_{G}\right)=\sigma_{\psi, \psi}$.

(2) $\operatorname{Res}_{B}^{G}\left(\psi \cdot \operatorname{St}_{G}\right)=\left(\sigma_{\psi, \psi}\right) \oplus\left(\psi^{2} \otimes \sigma\right)$.

(3) $\operatorname{Res}_{B}^{G} \pi_{\chi_{1}, \chi_{2}}=\left(\sigma_{\chi_{1}, \chi_{2}}\right) \oplus\left(\sigma_{\chi_{2}, \chi_{1}}\right) \oplus\left(\chi_{1} \chi_{2} \otimes \sigma\right)$.

(4) $\operatorname{Res}_{B}^{G} \pi_{\theta}=\left(\left.\theta\right|_{F^{\times}}\right) \otimes \sigma$.

Proof. See the table in [1, p. 87].

1.4. Let $L$ be the Galois field extension of $F$ of degree $n$. One knows that there exists the base-change map $\mathrm{Bc}_{L / F}: \operatorname{Irr}\left(\mathrm{GL}_{2}(F)\right) \longrightarrow \operatorname{Irr}\left(\mathrm{GL}_{2}(L)\right)(\mathrm{cf}$. [13]). Now we describe the explicit behaviour of this map in terms of the classification of the irreducible representations of the group $\mathrm{GL}_{2}$ in the cases $n=2,3$.

Theorem 1.5. (1) If $[L: F]=2$, then

(i) $\operatorname{Bc}_{L / F}\left(\pi_{\xi_{1}, \xi_{2}}\right)=\Pi_{\Xi_{1}, \Xi_{2}}$ where $\Xi_{i}=\xi_{i} \circ \mathrm{N}_{L / F}$ as characters of $L^{\times}$, for $i=1,2$;

(ii) $\mathrm{Bc}_{L / F}\left(\psi \cdot 1_{\mathrm{GL}_{2}(F)}\right)=\Psi \cdot 1_{\mathrm{GL}_{2}(L)}$ where $\Psi=\psi \circ \mathrm{N}_{L / F}$ as characters of $L^{\times}$;

(iii) $\mathrm{Bc}_{L / F}\left(\psi \cdot \mathrm{St}_{\mathrm{GL}_{2}(F)}\right)=\Psi \cdot \mathrm{St}_{\mathrm{GL}_{2}(L)}$ where $\Psi=\psi \circ \mathrm{N}_{L / F}$ as characters of $L^{\times}$;

(iv) $\mathrm{Bc}_{L / F}\left(\pi_{\theta}\right)=\prod_{\theta, \theta^{q}}$.

(2)If $[L: F]=3$, then

(i) $\mathrm{Bc}_{L / F}\left(\pi_{\xi_{1}, \xi_{2}}\right)=\Pi_{\Xi_{1}, \Xi_{2}}$ where $\Xi_{i}=\xi_{i} \circ \mathrm{N}_{L / F}$ as characters of $L^{\times}$,for $i=1,2$;

(ii) $\operatorname{Bc}_{L / F}\left(\psi \cdot 1_{\mathrm{GL}_{2}(F)}\right)=\Psi \cdot 1_{\mathrm{GL}_{2}(L)}$ where $\Psi=\psi \circ \mathrm{N}_{L / F}$ as characters of $L^{\times}$;

(iii) $\mathrm{Bc}_{L / F}\left(\psi \cdot \mathrm{St}_{\mathrm{GL}_{2}(F)}\right)=\Psi \cdot \mathrm{St}_{\mathrm{GL}_{2}(L)}$ where $\Psi=\psi \circ \mathrm{N}_{L / F}$ as characters of $L^{\times}$; 
(iv) $\mathrm{Bc}_{L / F}\left(\pi_{\theta}\right)=\Pi_{\Theta}$ where $\left[F_{1}: F\right]=2,\left[L_{1}: L\right]=2, L_{1} \supseteq F_{1}, \theta \in \operatorname{Irr}\left(F_{1}^{\times}\right)-\operatorname{Irr}\left(F^{\times}\right), \Theta \in \operatorname{Irr}\left(L_{1}^{\times}\right)-$ $\operatorname{Irr}\left(L^{\times}\right)$, and $\Theta=\theta \circ \mathrm{N}_{L_{1} / F_{1}}$ as characters of $L_{1}^{\times}$.

Proof. See [13, Section 4, p. 410—414].

1.5. As is known that one can generalize the above base-change map defined for other groups, called Shintani lifting or Shintani descent (e.g. [3]). In the article [7], Gyoja studied systematically Shintani lifting for connected linear algebraic groups. We will recall his certain results below. In addition, we also present one main result in [8] about the behaviour of the Weil representations with respect to Shintani lift.

Let $\bar{F}$ be a fixed algebraic closure of $F$ with Frobenius map $\sigma$. Let $\mathbf{G}$ be a connected linear algebraic group over $F$. Denote by $F_{i}$ the $\sigma^{i}$-fixed points of $\bar{F}$. Let $Y$ be a set on which there exists a $\sigma$-action; we denote the set of $\sigma$-fixed points by $Y_{\sigma}$. Denote by $\mathbf{G}\left(F_{i}\right)$ the $F_{i}$-geometric points of $\mathbf{G}$ and $C\left(\mathbf{G}\left(F_{i}\right)\right)$ the set of complex valued class functions of $\mathbf{G}\left(F_{i}\right)$. Fix a positive integer $m$. Via the map $\operatorname{Gal}(\bar{F} / F) \rightarrow \operatorname{Gal}\left(F_{m} / F\right)$, we view the Frobenius element $\sigma$ as one generator for the group $\operatorname{Gal}\left(F_{m} / F\right)$. For $0 \leq i \leq m-1$, let us denote by $\mathbf{G}\left(F_{m}\right) \rtimes \sigma^{i}$, the subset of the semi-direct product $\mathbf{G}\left(F_{m}\right) \rtimes \operatorname{Gal}\left(F_{m} / F\right)$ consisting of $\left(g, \sigma^{i}\right)$ 's for $g \in \mathbf{G}\left(F_{m}\right)$. In the article [7], following Kawanaka [9], Gyoja defined the norm maps $\mathrm{N}_{i}$ as follows:

$$
\begin{gathered}
N_{i}: \mathbf{G}\left(F_{m}\right) \rtimes \sigma^{i} \longrightarrow \mathbf{G}(\bar{F}) ; \\
{\left[x, \sigma^{i}\right] \longmapsto \alpha(x)\left(x \sigma^{i}(x) \cdots \sigma^{i\left(\frac{m}{d}-1\right)}(x)\right) \alpha(x)^{-1},}
\end{gathered}
$$

where $\alpha(x)$ is an element in $\mathbf{G}(\bar{F})$ such that $\alpha(x)^{-1} \sigma^{d}(\alpha(x))=x \sigma^{i}(x) \cdots \sigma^{i(t-1)}(x)$ and $d, t$ are the integers given by $d=(m, i)$ and $t i \equiv d(\bmod m)$. Here $(m, i)$ denotes the greatest common divisor of $m$ and $i$.

Each norm map $\mathrm{N}_{i}$ induces a bijection from the set of $\mathbf{G}\left(F_{m}\right)$-conjugacy classes of $\mathbf{G}\left(F_{m}\right) \rtimes \sigma^{i}$ onto the set of conjugacy classes of $\mathbf{G}\left(F_{m}\right)_{\sigma^{i}}=\mathbf{G}\left(F_{(m, i)}\right)$. Through $\mathbf{N}_{i}$, one defines the $i$-restriction map from $C\left(\mathbf{G}\left(F_{m}\right) \rtimes\right.$ $\left.\operatorname{Gal}\left(F_{m} / F\right)\right)$ to $C\left(\mathbf{G}\left(F_{(m, i)}\right)\right)_{\sigma}$ such that $(i-r e s(f)) \circ \mathbf{N}_{i}=\left.f\right|_{\mathbf{G}\left(F_{m}\right) \times \sigma^{i}}$ for any $f \in C\left(\mathbf{G}\left(F_{m}\right) \rtimes \operatorname{Gal}\left(F_{m} / F\right)\right)$.

Lemma 1.6. (i) For any $f, g \in C\left(\boldsymbol{G}\left(F_{m}\right)_{\sigma^{i}}\right)$, we have $\langle f, g\rangle_{\boldsymbol{G}\left(F_{m}\right)_{\sigma^{i}}}=\left\langle f \circ N_{i}, g \circ N_{i}\right\rangle_{\boldsymbol{G}\left(F_{m}\right) \times \sigma^{i}}$, where $\langle f, g\rangle_{\boldsymbol{G}\left(F_{(m, i)}\right)}:=$ $\frac{1}{\left|\boldsymbol{G}\left(F_{(m, i)}\right)\right|} \sum_{x \in \boldsymbol{G}\left(F_{(m, i)}\right)} f(x) \overline{g(x)}$ and $\left\langle f \circ N_{i}, g \circ N_{i}\right\rangle_{\boldsymbol{G}\left(F_{m}\right) \times \sigma^{i}}:=\frac{1}{\left|\boldsymbol{G}\left(F_{m}\right) \times \sigma^{i}\right|} \sum_{t \in \boldsymbol{G}\left(F_{m}\right)} f\left(N_{i}\left(\sigma^{i}, t\right)\right) \overline{g\left(N_{i}\left(\sigma^{i}, t\right)\right)}$.

(ii) The i-restrictions define an isomorphism: $\boldsymbol{C}\left(\boldsymbol{G}\left(F_{m}\right) \rtimes \operatorname{Gal}\left(F_{m} / F\right)\right) \simeq \oplus_{i=0}^{m-1} C\left(\boldsymbol{G}\left(F_{m}\right)_{\sigma^{i}}\right)_{\sigma}$.

Proof. See [7, p.11, Corollary 3.3 and p.1, Introduction].

Lemma 1.7. Let $\boldsymbol{H}$ be a connected closed subgroup of $\boldsymbol{G}$ defined over $F$. Then the following diagram is commutative

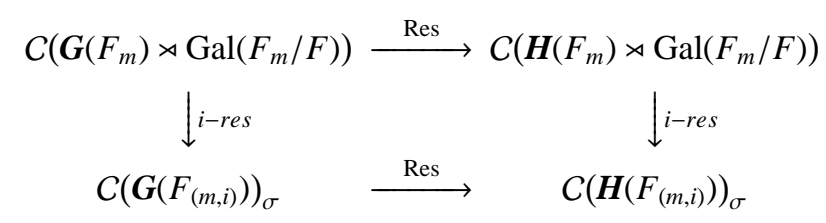

Proof. See [7, p. 12, Lemma 3.6] .

Now let $V,\langle$,$\rangle be a symplectic space over F$ and let $\mathbf{G}=\mathbf{G S p}_{V}$ be the algebraic group of symplectic similitudes of $(V,\langle\rangle$,$) . For 0 \leq i \leq m-1$, write $\Xi_{F_{(m, i)}}$ for the Weil representation of $\mathbf{G}\left(F_{(m, i)}\right)$

Proposition 1.8. There exists a unique representation $\widetilde{\Xi_{F_{m}}}$ of $\boldsymbol{G}\left(F_{m}\right) \rtimes \operatorname{Gal}\left(F_{m} / F\right)$ such that $i$-res $\left(\widetilde{\Xi_{F_{m}}}\right)=\Xi_{F_{(m, i)}}$ for $0 \leq i \leq m-1$.

Proof. See [8, Theorem 4.2].

2. The decomposition of the WeIl Representation of $\mathrm{GL}_{2}(F) \times \mathrm{GL}_{2}(F) \times \mathrm{GL}_{2}(F)$

2.1. We give some notations and formulate the mainly studied representation in this section.

In this section, we use the following notations: $G=\mathrm{GL}_{2}(F), H=G \times G, B=\left\{\left(\begin{array}{ll}a & b \\ 0 & d\end{array}\right) \in G\right\}, N=\left\{\left(\begin{array}{ll}1 & b \\ 0 & 1\end{array}\right) \in\right.$ $G\}, T=\left\{\left(\begin{array}{ll}a & 0 \\ 0 & d\end{array}\right) \in G\right\}, Z=\left\{\left(\begin{array}{ll}a & 0 \\ 0 & a\end{array}\right) \in G\right\} ; h(r)=\left(\begin{array}{cc}r & 0 \\ 0 & r^{-1}\end{array}\right), u(b)=\left(\begin{array}{ll}1 & b \\ 0 & 1\end{array}\right), h^{\prime}(t)=\left(\begin{array}{ll}1 & 0 \\ 0 & t\end{array}\right), \omega^{\prime}=\left(\begin{array}{ll}0 & 1 \\ 1 & 0\end{array}\right)$, 
$\omega=\left(\begin{array}{cc}0 & 1 \\ -1 & 0\end{array}\right)$ in $G, S_{3}=$ the permutation group of 3 variables.

Let $V$ be a vector space over $F$ of dimension 2, endowed with a non-degenerate symplectic form $\langle$,$\rangle . Let \left\{e_{1}, e_{2}\right\}$ be a symplectic basis of $V$ i.e. $\left\langle e_{1}, e_{2}\right\rangle=1,\left\langle e_{2}, e_{1}\right\rangle=-1$. We attach the vector space $V^{\otimes 3}=V \otimes_{F} V \otimes_{F} V$ with the natural symplectic form $\langle,\rangle \otimes\langle,\rangle \otimes\langle$,$\rangle , so there exists a homomorphism p$ from $(\mathrm{GSp}(V) \times \mathrm{GSp}(V) \times \mathrm{GSp}(V)) \rtimes S S_{3}$ to $\mathrm{GSp}\left(V^{\otimes 3}\right)$. The above group $S_{3}$ acts on $V \otimes V \otimes V$ by permutations. By the fixed basis $\left\{e_{1}, e_{2}\right\}$ of $\mathrm{V}$ and $\left\{e_{i} \otimes e_{j} \otimes e_{k} \mid 1 \leq i, j, k \leq 2\right\}$ of $V^{\otimes 3}$, we could identify the group $G$ with $\operatorname{GL}(V)$, and the group $\operatorname{GSp}_{8}(F)$ with $\operatorname{GSp}\left(V^{\otimes 3}\right)$.

Let $\rho$ be the Weil representation of $\operatorname{GSp}\left(V^{\otimes 3}\right)$. Through the above morphism $p$, we get a representation $\pi^{\prime}$ of the $\operatorname{group}(\mathrm{GSp}(V) \times \mathrm{GSp}(V) \times \mathrm{GSp}(V)) \rtimes S_{3}$. Let $\pi$ denote the restriction of $\pi^{\prime}$ to $\mathrm{GSp}(V) \times \mathrm{GSp}(V) \times \mathrm{GSp}(V)$. Write ${ }_{+} V^{\otimes 3}=\left\{x \in V^{\otimes 3} \mid x \in F e_{1} \otimes V \otimes V\right\}$ and $\_V^{\otimes 3}=\left\{y \in V^{\otimes 3} \mid y \in F e_{2} \otimes V \otimes V\right\}$. Every element $y \in{ }_{-} V^{\otimes 3}$ has the form $y=\sum_{j, k=1}^{2} a_{j, k} e_{2} \otimes e_{j} \otimes e_{k}$, which corresponds to a matrix $m=\left(\begin{array}{ll}a_{11} & a_{12} \\ a_{21} & a_{22}\end{array}\right)$. So we could identify $V^{\otimes 3}$ with the matrix ring $M_{2}(F)$ as vector space over $F$. The representation $\pi$ of $G \times G \times G$ can be realized in the vector space $W=\mathbb{C}\left[M_{2}(F) \times X_{F}\right]$ of complex functions on $M_{2}(F) \times X_{F}$.

Proposition 2.1. The representation $(\pi, G \times G \times G, W)$ is given by the following formulas:

$$
\begin{gathered}
(\pi[h(a), 1,1] f)(m, \psi)=f(a m, \psi), \\
(\pi[u(b), 1,1] f)(m, \psi)=\psi(b \operatorname{det}(m)) f(m, \psi), \\
\left(\pi\left[h^{\prime}(t), 1,1\right] f\right)(m, \psi)=f\left(m, \psi^{t^{-1}}\right), \\
(\pi[\omega, 1,1] f)(m, \psi)=q^{-2} \sum_{n \in M_{2}(F)} \psi(B(m, n)) f(n, \psi), \\
\left(\pi\left[1, g_{2}, g_{3}\right] f\right)(m, \psi)=f\left(\operatorname{det}\left(g_{2} g_{3}\right) g_{2}^{-1} m\left(g_{3}^{-1}\right)^{t}, \psi^{\operatorname{det}\left(g_{2} g_{3}\right)^{-1}}\right),
\end{gathered}
$$

where $g_{2}, g_{3} \in G, m \in M_{2}(F), g_{3}^{t}=$ the transpose of $g_{3}, B(m, n)=m_{11} n_{22}+m_{22} n_{11}-m_{12} n_{21}-m_{21} n_{12}$ for $m=$ $\left(\begin{array}{ll}m_{11} & m_{12} \\ m_{21} & m_{22}\end{array}\right), n=\left(\begin{array}{ll}n_{11} & n_{12} \\ n_{21} & n_{22}\end{array}\right) \in M_{2}(F)$.

Proof. 2.1 - 2.4 come directly from the formulas (1.1) (1.4) in Section 1 Consider now the formula (2.5). Recall, for $g \in G, g \cdot e_{1}:=\left(e_{1}, e_{2}\right) g\left(\begin{array}{l}1 \\ 0\end{array}\right)$ and $g \cdot e_{2}:=\left(e_{1}, e_{2}\right) g\left(\begin{array}{l}0 \\ 1\end{array}\right)$. By the fixed basis $\left\{e_{2} \otimes e_{j} \otimes e_{k} \mid 1 \leq j, k \leq 2\right\}$, we obtain $g_{2} \otimes g_{3} \cdot m:=g_{2} m g_{3}^{t}$ for $g_{2}, g_{3} \in G, m \in M_{2}(F)$. Then, by (1.1), (1.4) in Section 1, we have

$$
\begin{gathered}
\left(\pi\left[1, g_{2}, g_{3}\right] f\right)(m, \psi)=\rho\left(\left(\begin{array}{cc}
1 & 0 \\
0 & \operatorname{det}\left(g_{2} g_{3}\right)
\end{array}\right)\left(\begin{array}{cc}
g_{2} \otimes g_{3} & 0 \\
0 & \operatorname{det}\left(g_{2} g_{3}\right)^{-1} g_{2} \otimes g_{3}
\end{array}\right)\right) f(m, \psi) \\
=\rho\left(\left(\begin{array}{cc}
g_{2} \otimes g_{3} & 0 \\
0 & \operatorname{det}\left(g_{2} g_{3}\right)^{-1} g_{2} \otimes g_{3}
\end{array}\right)\right) f\left(m, \psi^{\operatorname{det}\left(g_{2} g_{3}\right)^{-1}}\right) \\
=\chi_{q}^{+}\left(\operatorname{det}\left(g_{2} \otimes g_{3}\right)^{2}\right) f\left(g_{2}^{-1} \otimes g_{3}^{-1} \operatorname{det}\left(g_{2} g_{3}\right) \cdot m, \psi^{\operatorname{det}\left(g_{2} g_{3}\right)^{-1}}\right)=f\left(\operatorname{det}\left(g_{2} g_{3}\right) g_{2}^{-1} m\left(g_{3}^{-1}\right)^{t}, \psi^{\operatorname{det}\left(g_{2} g_{3}\right)^{-1}}\right) .
\end{gathered}
$$

2.2. To decompose the representation $\pi$, it involves to describe the $1 \times G \times G$-invariant part of the vector space $W$.

We consider the set $\mathcal{S}=\left\{\left(\pi_{1}, \pi_{2}\right) \mid\right.$ for $i=1,2,\left(\pi_{i}, V_{i}\right) \in \operatorname{Rep}(G)$ such that $\left.\left.\left(\pi_{1} \otimes \pi_{2}\right)\right|_{Z}=\operatorname{Id}_{V_{1} \times V_{2}}\right\}$. For each pair $\left(\pi_{1}, \pi_{2}\right) \in \mathcal{S}$, it determines a representation $\left(\pi_{1} \otimes \pi_{2}, V_{1} \otimes V_{2}\right)$ of the group $H$. We write $\operatorname{Irr}_{0}(H)$ for the set of the isomorphism classes of all irreducible representations $\left(\pi_{1} \otimes \pi_{2}, V_{1} \otimes V_{2}\right)$ of $H$ for which $\left(\pi_{1}, \pi_{2}\right) \in \mathcal{S}$.

Now we concentrate on the decomposition of the representation $(\pi, G \times G \times G, W)$. Following the method in [1], we first associate a representation $\left(\pi_{0}^{\prime}, G, W\left[\pi_{1} \otimes \pi_{2}\right]\right)$ to any representation $\pi_{1} \otimes \pi_{2} \in \operatorname{Irr}_{0}(H)$, where the vector space $W\left[\pi_{1} \otimes \pi_{2}\right]$ consists of all functions $f: M_{2}(F) \times X_{F} \longrightarrow V_{1} \otimes V_{2}$ such that

$$
f\left(\operatorname{det}\left(g_{1}^{-1} g_{2}^{-1}\right) g_{1} m g_{2}^{t}, \psi^{\operatorname{det}\left(g_{1} g_{2}\right)}\right)=\left(\pi_{1}\left(g_{1}\right) \otimes \pi_{2}\left(g_{2}\right)\right) f(m, \psi),
$$


for $\left(g_{1}, g_{2}\right) \in H, m \in M_{2}(F), \psi \in X_{F}$, and the action of $G$ on $W\left[\pi_{1} \otimes \pi_{2}\right]$ is given by the formulas (2.1) - (2.4) in Proposition 2.1.

Proposition 2.2. For the representation $(\pi, G \times G \times G, W)$, we have

$$
W=\bigoplus_{\pi_{1} \otimes \pi_{2} \in I r r_{0}(H)} W\left[\pi_{1} \otimes \pi_{2}\right] \otimes \check{V}_{1} \otimes \check{V}_{2}
$$

Proof. Since the representation $(\pi, W)$ of $G \times G \times G$ is semi-simple and arises from the restriction of $\rho$, we have

$$
W=\oplus_{\check{\pi}_{1} \otimes \check{\pi}_{2} \in \operatorname{Irr}_{0}(H)} W_{\check{\pi}_{1} \otimes \check{\pi}_{2}} \otimes \check{V}_{1} \otimes \check{V}_{2} .
$$

Here $W_{\check{\pi}_{1} \otimes \check{\pi}_{2}}$ is the greatest $\check{\pi}_{1} \otimes \check{\pi}_{2}$-isotypic quotient of $W$ (cf. [10, p. 46, III.4]). Note that

$$
W_{\check{\pi}_{1} \otimes \check{\pi}_{2}} \simeq\left[W \otimes\left(V_{1} \otimes V_{2}\right)\right]^{G \times G} \simeq W\left[V_{1} \otimes V_{2}\right]
$$

as $G$-module. In (2.7), we treat $W$ as $G \times G$-module via the embedding $G \times G \simeq 1 \times G \times G \hookrightarrow G \times G \times G$.

Recall the Cartan involution: $\theta: G \longrightarrow G ; g \longmapsto\left(g^{t}\right)^{-1}$. It is well-known that $\sigma \simeq(\sigma \circ \theta)^{\vee}$ for $\sigma \in \operatorname{Irr}(G)$. Let $\left(\left[\pi_{1}, \pi_{2} \circ t\right], \operatorname{Hom}_{\mathbb{C}}\left(V_{2}, V_{1}\right)\right)$ be a representation of $G \times G$, defined by

$$
\left[\pi_{1}, \pi_{2} \circ t\right]\left(g_{1}, g_{2}\right)(\varphi)=\pi_{1}\left(g_{1}\right) \circ \varphi \circ \pi_{2}\left(g_{2}^{t}\right), \quad g_{1}, g_{2} \in G, \varphi \in \operatorname{Hom}_{\mathbb{C}}\left(V_{2}, V_{1}\right) .
$$

Define an isomorphism of vector spaces:

$$
\begin{gathered}
\lambda: V_{1} \otimes V_{2}^{\star} \longrightarrow \operatorname{Hom}_{\mathbb{C}}\left(V_{2}, V_{1}\right) ; \\
v_{1} \otimes v_{2}^{\star} \longrightarrow\left(\varphi_{v_{1} \otimes v_{2}^{\star}}: v_{2} \longmapsto\left\langle v_{2}^{\star}, v_{2}\right\rangle v_{1}\right) .
\end{gathered}
$$

It can be checked that $\lambda$ defines an intertwining operator between $\left(\pi_{1} \otimes\left(\pi_{2} \circ \theta\right)^{\vee}, V_{1} \otimes V_{2}^{\star}\right)$ and $\left(\left[\pi_{1}, \pi_{2} \circ\right.\right.$ $\left.t], \operatorname{Hom}_{\mathbb{C}}\left(V_{2}, V_{1}\right)\right)$. To simplify calculation, we replace $\left(\pi_{1} \otimes \pi_{2}, V_{1} \otimes V_{2}\right)$ with $\left(\left[\pi_{1}, \pi_{2} \circ t\right], \operatorname{Hom}_{\mathbb{C}}\left(V_{2}, V_{1}\right)\right)$ in (2.7), and get an isomorphic representation of $\left(\pi_{0}^{\prime}, G, W\left[\pi_{1} \otimes \pi_{2}\right]\right)$, say $\left(\pi_{0}, G, W\left[\pi_{1}, \pi_{2}\right]\right)$, where $W\left[\pi_{1}, \pi_{2}\right]$ is a vector space over $\mathbb{C}$ consisting of all functions $f: M_{2}(F) \times X_{F} \longrightarrow \operatorname{Hom}_{\mathbb{C}}\left(V_{2}, V_{1}\right)$ such that

$$
f\left(\operatorname{det}\left(g_{1}^{-1} g_{2}^{-1}\right) g_{1} m g_{2}^{t}, \psi^{\operatorname{det}\left(g_{1} g_{2}\right)}\right)=\pi_{1}\left(g_{1}\right) \circ f(m, \psi) \circ \pi_{2}\left(g_{2}^{t}\right), \quad g_{1}, g_{2} \in G,
$$

and the action of $G$ on $W\left[\pi_{1}, \pi_{2}\right]$ arises naturally from the above formulas 2.1 - 2.4 in Proposition 2.1.

2.3. We continue the above discussion, and determine the dimension of the vector space $W\left[\pi_{1}, \pi_{2}\right]$.

For $\pi_{1} \otimes \pi_{2} \in \operatorname{Irr}_{0}(H)$, we write $W\left[\pi_{1}, \pi_{2}\right](\xi)=\left\{f(\xi) \mid f \in W\left[\pi_{1}, \pi_{2}\right], \xi \in M_{2}(F) \times X_{F}\right\}$. Now we define an $H$-action on the set $M_{2}(F) \times X_{F}$ as follows:

$$
\left(g_{1}, g_{2}\right)(m, \psi):=\left(\operatorname{det}\left(g_{1}^{-1} g_{2}^{-1}\right) g_{1} m g_{2}^{t}, \psi^{\operatorname{det}\left(g_{1} g_{2}\right)}\right)
$$

where $\left(g_{1}, g_{2}\right) \in H, \psi \in X_{F}, m \in M_{2}(F)$. It is observed that $W\left[\pi_{1}, \pi_{2}\right](\xi)=\operatorname{Fix}_{\mathrm{Hom}_{\mathbb{C}}\left(V_{2}, V_{1}\right)}\left(\operatorname{Stab}_{H}(\xi)\right)$ for $\xi \in$ $M_{2}(F) \times X_{F}$, more precisely

$$
W\left[\pi_{1}, \pi_{2}\right](\xi)=\left\{\varphi: V_{2} \longrightarrow V_{1} \mid \pi_{1}\left(g_{1}\right) \circ \varphi=\varphi \circ \pi_{2}\left(\left(g_{2}^{t}\right)^{-1}\right), \quad\left(g_{1}, g_{2}\right) \in \operatorname{Stab}_{H}(\xi)\right\} .
$$

Let us determine the $H$-orbits in $M_{2}(F) \times X_{F}$. They are of the following three kinds:

(i) Orbit $\left\{\xi_{a}\right\}$, where $\xi_{a}=\left(\left(\begin{array}{ll}1 & 0 \\ 0 & 1\end{array}\right), \phi^{a}\right) \quad$ for any $a \in F^{\times}$;

(ii) Orbit $\{\eta\}$, where $\eta=\left(\left(\begin{array}{ll}1 & 0 \\ 0 & 0\end{array}\right), \phi\right)$;

(iii) Orbit $\{\delta\}$, where $\delta=\left(\left(\begin{array}{ll}0 & 0 \\ 0 & 0\end{array}\right), \phi\right)$.

By straightforward calculation, the corresponding stabilizer of the given representative element in each orbit has the following form:

(i) $\operatorname{Stab}_{H}\left(\xi_{a}\right)=\left\{\left(g,\left(g^{-1}\right)^{t}\right) \mid g \in G\right\}$;

(ii) $\operatorname{Stab}_{H}(\eta)=\left\{\left(s n_{1}, s^{-1} n_{2}\right) \mid s \in T, n_{1}, n_{2} \in N\right\}$;

(iii) $\operatorname{Stab}_{H}(\delta)=\left\{\left(g_{1}, g_{2}\right) \mid g_{1}, g_{2} \in G\right.$ and $\left.\operatorname{det}\left(g_{1} g_{2}\right)=1\right\}$.

To obtain the dimension of the vector space $W\left[\pi_{1}, \pi_{2}\right]$, we state the lemma: 
Lemma 2.3. (1) $W\left[\pi_{1}, \pi_{2}\right]\left(\xi_{a}\right) \neq 0$ if and only if $\pi_{1} \simeq \pi_{2}$, in which case $\operatorname{dim}_{\mathbb{C}} W\left[\pi_{1}, \pi_{2}\right]\left(\xi_{a}\right)=1$; (2) $W\left[\pi_{1}, \pi_{2}\right](\eta)=0$ except the following cases:
(a) $\operatorname{dim}_{\mathbb{C}} W\left[\pi_{\chi_{1}, \chi_{2}}, \pi_{\chi_{1}, \chi_{2}}\right](\eta)=2$,
(b) $\operatorname{dim}_{\mathbb{C}} W\left[\psi \cdot 1_{G}, \psi \cdot 1_{G}\right](\eta)=1$,
(c) $\operatorname{dim}_{\mathbb{C}} W\left[\psi \cdot \mathrm{St}_{G}, \psi \cdot \mathrm{St}_{G}\right](\eta)=1$,
(d) $\operatorname{dim}_{\mathbb{C}} W\left[\psi \cdot 1_{G}, \psi \cdot \mathrm{St}_{G}\right](\eta)=1$,
(e) $\operatorname{dim}_{\mathbb{C}} W\left[\psi \cdot \mathrm{St}_{G}, \psi \cdot 1_{G}\right](\eta)=1$,

for the characters $\chi_{1} \neq \chi_{2}, \psi$ of $F^{\times}$;

(3) $W\left[\pi_{1}, \pi_{2}\right](\delta)=0$ except $\pi_{1}=\pi_{2}=\psi \cdot 1_{G}$, in that case $\operatorname{dim}_{\mathbb{C}} W\left[\psi \cdot 1_{G}, \psi \cdot 1_{G}\right](\delta)=1$ for any character $\psi$ of $F^{\times}$.

Proof. 1) By the formula (2.10), the vector space $W\left[\pi_{1}, \pi_{2}\right]\left(\xi_{a}\right)$ consists of the functions $\varphi: V_{2} \longrightarrow V_{1}$ such that $\pi_{1}\left(g_{1}\right) \circ \varphi=\varphi \circ \pi_{2}\left(\left(g_{2}^{-1}\right)^{t}\right)$ for $\left(g_{1}, g_{2}\right) \in \operatorname{Stab}_{H}\left(\xi_{a}\right)$. Hence $W\left[\pi_{1}, \pi_{2}\right]\left(\xi_{a}\right)$ is isomorphic to $\operatorname{Hom}_{G}\left(V_{2}, V_{1}\right)$.

2) Note that $W\left[\pi_{1}, \pi_{2}\right](\eta) \simeq \operatorname{Hom}_{T}\left(V_{2}^{N}, V_{1}^{N}\right)$. Therefore $W\left[\pi_{1}, \pi_{2}\right](\eta)=0$ unless $\pi_{1}, \pi_{2}$ both are induced representations. Consider the induced representation $\left(\pi_{\chi_{1}, \chi_{2}}, V\right)=\operatorname{Ind}_{B}^{G}\left(\chi_{1} \otimes \chi_{2}\right)$ for $\chi_{1}, \chi_{2} \in \operatorname{Irr}\left(F^{\times}\right)$. The vector space $V^{N}$ is generated by the following two functions $f_{\chi_{1}, \chi_{2}}$ and $g_{\chi_{1}, \chi_{2}}$, where

1. the support of $f_{\chi_{1}, \chi_{2}}$ belongs to $B$, and $f_{\chi_{1}, \chi_{2}}(t n)=\chi_{1} \otimes \chi_{2}(t)$ for $t \in T, n \in N$;

2. the support of $g_{\chi_{1}, \chi_{2}}$ belongs to $B \omega^{\prime} N$, and $g_{\chi_{1}, \chi_{2}}\left(t n_{1} \omega^{\prime} n_{2}\right)=\chi_{1} \otimes \chi_{2}(t)$ for $t \in T, n_{1}, n_{2} \in N$.

The action of $T$ on $V^{N}=\left\{f_{\chi_{1}, \chi_{2}}, g_{\chi_{1}, \chi_{2}}\right\}$ is simply given by the formulas:

$$
t \cdot f_{\chi_{1}, \chi_{2}}=\chi_{1} \otimes \chi_{2}(t) f_{\chi_{1}, \chi_{2}} \quad \text { and } \quad t \cdot g_{\chi_{1}, \chi_{2}}=\chi_{2} \otimes \chi_{1}(t) g_{\chi_{1}, \chi_{2}}, \quad t \in T .
$$

Thus, $\operatorname{dim}_{\mathbb{C}} \operatorname{Hom}_{T}\left(\pi_{\chi_{1}, \chi_{2}}^{N}, \pi_{\chi_{1}, \chi_{2}}^{N}\right)=2$ for $\chi_{1} \neq \chi_{2} \in \operatorname{Irr}\left(F^{\times}\right)$, and (a) follows. On the other hand $\operatorname{dim}_{\mathbb{C}} \operatorname{Hom}_{T}\left(\pi_{\psi, \psi}^{N}, \pi_{\psi, \psi}^{N}\right)=4$ for $\psi \in \operatorname{Irr}\left(F^{\times}\right)$. Clearly $\left(\operatorname{St}_{G}\right)^{N}=\mathbb{C}\left(q f_{1_{G}, 1_{G}}-g_{1_{G}, 1_{G}}\right)$ and $\left(1_{G}\right)^{N}=\mathbb{C}\left(f_{1_{G}, 1_{G}}+g_{1_{G}, 1_{G}}\right)$. So (b) and (c) hold and $\operatorname{dim}_{\mathbb{C}} W\left[\psi \cdot 1_{G}, \psi \cdot \operatorname{St}_{G}\right](\eta)=\operatorname{dim}_{\mathbb{C}} W\left[\psi \cdot \mathrm{St}_{G}, \psi \cdot 1_{G}\right](\eta)=1$.

$3)$ By the formula (2.10), $W\left[\pi_{1}, \pi_{2}\right](\delta)$ consists of the functions $\varphi: V_{2} \longrightarrow V_{1}$ such that $\pi_{1}\left(g_{1}\right) \circ \varphi=\varphi \circ \pi_{2}\left(\left(g_{2}^{-1}\right)^{t}\right)$ for $\left(g_{1}, g_{2}\right) \in \operatorname{Stab}_{H}(\delta)$. Since $\operatorname{Stab}_{H}(\delta)=\left\{\left(g_{1}, g_{2}\right) \mid g_{1}, g_{2} \in G\right.$ and $\left.\operatorname{det}\left(g_{1} g_{2}\right)=1\right\}$, we know $\varphi=0$ except that $\pi_{1}=\psi_{1} \circ$ det, $\quad \pi_{2}=\psi_{2} \circ$ det, in which case, $W\left[\pi_{1}, \pi_{2}\right](\delta) \simeq \operatorname{Hom}_{F^{\times}}\left(\psi_{2}, \psi_{1}\right)$, and we get the result.

Corollary 2.4. For any irreducible representation $\pi_{1} \otimes \pi_{2} \in \operatorname{Irr}_{0}(H)$, the dimension of the representation $\left(\pi_{0}, G, W\left[\pi_{1}, \pi_{2}\right]\right)$ has the following form:

(i) $\operatorname{dim}_{\mathbb{C}} W\left[\pi_{\chi_{1}, \chi_{2}}, \pi_{\chi_{1}, \chi_{2}}\right]=q+1$,

(ii) $\operatorname{dim}_{\mathbb{C}} W\left[\psi \cdot \mathrm{St}_{G}, \psi \cdot \mathrm{St}_{G}\right]=q$,

(iii) $\operatorname{dim}_{\mathbb{C}} W\left[\psi \cdot 1_{G}, \psi \cdot 1_{G}\right]=q+1$,

(iv) $\operatorname{dim}_{\mathbb{C}} W\left[\pi_{\theta}, \pi_{\theta}\right]=q-1$,

(v) $\operatorname{dim}_{\mathbb{C}} W\left[\psi \cdot \mathrm{St}_{G}, \psi \cdot 1_{G}\right]=1$,

(vi) $\operatorname{dim}_{\mathbb{C}} W\left[\psi \cdot 1_{G}, \psi \cdot \mathrm{St}_{G}\right]=1$,

for the characters $\chi_{1} \neq \chi_{2}, \psi$ of $F^{\times}$, the regular character $\theta$ of $E^{\times}$. And the above lists are all the representations $\pi_{1} \otimes \pi_{2} \in \operatorname{Irr}_{0}(H)$, such that $W\left[\pi_{1}, \pi_{2}\right] \neq 0$.

Proof. Note that $\operatorname{dim}_{\mathbb{C}} W\left[\pi_{1}, \pi_{2}\right]=\sum_{a \in F^{\times}} \operatorname{dim}_{\mathbb{C}} W\left[\pi_{1}, \pi_{2}\right]\left(\xi_{a}\right)+\operatorname{dim}_{\mathbb{C}} W\left[\pi_{1}, \pi_{2}\right](\eta)+\operatorname{dim}_{\mathbb{C}} W\left[\pi_{1}, \pi_{2}\right](\delta)$, so the corollary results immediately from above Lemma 2.3

2.4. We have already calculated the dimension of the vector space $W\left[\pi_{1}, \pi_{2}\right]$, and it suffices to prove the main theorem in this section.

Theorem 2.5. For $(\pi, G \times G \times G, W)$, we have:

$$
\pi \simeq \bigoplus_{\sigma \in \operatorname{Irr}(G)}(\sigma \otimes \sigma \otimes \sigma) \oplus \bigoplus_{\psi \in \operatorname{Irr}\left(F^{\times}\right)}\left(\left(\psi \cdot \mathrm{St}_{G} \otimes \psi \cdot 1_{G} \otimes \psi \cdot 1_{G}\right) \oplus\left(\psi \cdot 1_{G} \otimes \psi \cdot \mathrm{St}_{G} \otimes \psi \cdot 1_{G}\right) \oplus\left(\psi \cdot 1_{G} \otimes \psi \cdot 1_{G} \otimes \psi \cdot \mathrm{St}_{G}\right)\right) .
$$

Proof. Since $\pi=\operatorname{Res}_{G \times G \times G}^{(G \times G \times G) \rtimes S_{3}} \pi^{\prime}$, by Clifford theory, the representation $\pi$ is the direct sum of the following three kinds of representations:

(1) $\tau_{0} \otimes \tau_{0} \otimes \tau_{0}$ for $\tau_{0} \in \operatorname{Irr}(G)$;

(2) $\tau_{1} \otimes \tau_{1} \otimes \tau_{2}+\tau_{1} \otimes \tau_{2} \otimes \tau_{1}+\tau_{2} \otimes \tau_{1} \otimes \tau_{1}$ for $\tau_{1} \neq \tau_{2} \in \operatorname{Irr}(G)$; 
(3) $\tau_{0}^{\prime} \otimes \tau_{1}^{\prime} \otimes \tau_{2}^{\prime}+\tau_{0}^{\prime} \otimes \tau_{2}^{\prime} \otimes \tau_{1}^{\prime}+\tau_{1}^{\prime} \otimes \tau_{0}^{\prime} \otimes \tau_{2}^{\prime}+\tau_{1}^{\prime} \otimes \tau_{2}^{\prime} \otimes \tau_{0}^{\prime}+\tau_{2}^{\prime} \otimes \tau_{0}^{\prime} \otimes \tau_{1}^{\prime}+\tau_{2}^{\prime} \otimes \tau_{1}^{\prime} \otimes \tau_{0}^{\prime}$ for three different representations $\tau_{0}^{\prime}, \tau_{1}^{\prime}, \tau_{2}^{\prime}$ in $\operatorname{Irr}(G)$.

Comparing with the results in Corollary 2.4 gives the theorem.

\section{The Decomposition of the WeIL Representation of $\mathrm{GL}_{2}(F) \times \mathrm{GL}_{2}(E)$}

3.1. We first give some notations and formulate the representation concerned in this section.

In this section, we use the following notations: $G=\mathrm{GL}_{2}(F), B=\left\{\left(\begin{array}{ll}a & b \\ 0 & d\end{array}\right) \in G\right\}, N=\left\{\left(\begin{array}{ll}1 & b \\ 0 & 1\end{array}\right) \in G\right\}, T=$ $\left\{\left(\begin{array}{ll}a & 0 \\ 0 & d\end{array}\right) \in G\right\}, Z=\left\{\left(\begin{array}{ll}a & 0 \\ 0 & a\end{array}\right) \in G\right\} ; H=\mathrm{GL}_{2}(E), B^{\prime}=\left\{\left(\begin{array}{ll}a & b \\ 0 & d\end{array}\right) \in H\right\}, N^{\prime}=\left\{\left(\begin{array}{ll}1 & b \\ 0 & 1\end{array}\right) \in H\right\}, T^{\prime}=\left\{\left(\begin{array}{ll}a & 0 \\ 0 & d\end{array}\right) \in\right.$ $H\}, Z^{\prime}=\left\{\left(\begin{array}{ll}a & 0 \\ 0 & a\end{array}\right) \in H\right\} ; h(r)=\left(\begin{array}{cc}r & 0 \\ 0 & r^{-1}\end{array}\right), u(b)=\left(\begin{array}{cc}1 & b \\ 0 & 1\end{array}\right), h^{\prime}(t)=\left(\begin{array}{cc}1 & 0 \\ 0 & t\end{array}\right), \omega^{\prime}=\left(\begin{array}{cc}0 & 1 \\ 1 & 0\end{array}\right), \omega=\left(\begin{array}{cc}0 & 1 \\ -1 & 0\end{array}\right)$ in $G$ or $H$; $\operatorname{Gal}(E / F)=\langle\sigma\rangle$.

If $h \in H$ or $M_{2}(E)$, we will denote its conjugate by $h^{\sigma}$ or $\bar{h}$, its transpose by $h^{t}$, and let $h^{\star}:=\bar{h}^{t}$.

Let $V$ be a vector space over $F$ of dimension 2, endowed with a symplectic form $\langle$,$\rangle . Let \left\{e_{1}, e_{2}\right\}$ be a symplectic basis of $V$. Consider the $E$-vector space $V_{E}=E \otimes_{F} V$, endowed with the symplectic form $\langle,\rangle_{V_{E}}$ induced from $V$. Define a $\operatorname{Gal}(E / F)$-action on $V_{E}$ by

$$
\operatorname{Gal}(E / F) \times E \otimes_{F} V \longrightarrow E \otimes_{F} V ;\left(\sigma, \sum_{i} t_{i} \otimes e_{i}\right) \longmapsto \sum_{i} t_{i}^{\sigma} \otimes e_{i}
$$

Now let $\mathbb{W}=V_{E} \otimes_{E} V_{E}$ endowed with the symmetric form $\langle,\rangle_{\mathbb{W}}=\langle,\rangle_{V_{E}} \otimes\langle,\rangle_{V_{E}}$. On $\mathbb{W}$, we consider the twisted Galois action defined by

$$
\operatorname{Gal}(E / F) \times \mathbb{W} \longrightarrow \mathbb{W} ;\left(\sigma, w=\sum_{i} u_{i} \otimes v_{i}\right) \longmapsto{ }^{\sigma} w=\sum_{i} v_{i}^{\sigma} \otimes u_{i}^{\sigma}
$$

We will let $\mathbb{W}_{0}$ denote the set $\left\{\left.w \in \mathbb{W}\right|^{\sigma} w=w\right\}$. It can be checked that the restriction of $\langle,\rangle_{\mathbb{W}}$ to $\mathbb{W}_{0}$ defines an $F$-symmetric form, denoted by $(,)_{\mathbb{W}_{0}}$. Let $q$ be its associative quadratic form given by

$$
\left(w_{0}, w_{0}^{\prime}\right)_{\mathbb{W}_{0}}=q\left(w_{0}+w_{0}^{\prime}\right)-q\left(w_{0}\right)-q\left(w_{0}^{\prime}\right), \quad w_{0}, w_{0}^{\prime} \in \mathbb{W}_{0} .
$$

By calculation, each $w_{0} \in \mathbb{W}_{0}$ may be expressed in the form

$$
w_{0}=x e_{1} \otimes e_{1}+\alpha e_{1} \otimes e_{2}+\bar{\alpha} e_{2} \otimes e_{1}+y e_{2} \otimes e_{2} \text { for } x, y \in F, \alpha \in E .
$$

Every element $w_{0}$ corresponds to a matrix $\left(\begin{array}{cc}x & \alpha \\ \alpha & y\end{array}\right)$. So we can identify $\mathbb{W}_{0}$ with $M=\left\{\left(\begin{array}{ll}x & \alpha \\ \alpha & y\end{array}\right) \mid x, y \in F, \alpha \in E\right\}$. The symmetric form $q$ is transferred as $q(m)=\operatorname{det}(m)$ for $m \in M$.

Let $\mathrm{GO}(\mathbb{W})$ denote the group of symmetric similitudes of $\left(\mathbb{W},\langle,\rangle_{\mathbb{W}}\right)$. By the definition of $\mathbb{W}$, there exists a morphism of groups: $\mathrm{GL}\left(V_{E}\right) \times \mathrm{GL}\left(V_{E}\right) \longrightarrow \mathrm{GO}(\mathbb{W})$. We define a twisted Galois action of $\operatorname{Gal}(E / F)$ on $\operatorname{GL}\left(V_{E}\right) \times$ GL $\left(V_{E}\right)$ by

$$
\mathrm{Gal}(E / F) \times\left(\mathrm{GL}\left(V_{E}\right) \times \mathrm{GL}\left(V_{E}\right)\right) \longrightarrow \mathrm{GL}\left(V_{E}\right) \times \mathrm{GL}\left(V_{E}\right) ; h=\left(g_{1}, g_{2}\right) \longmapsto{ }^{\sigma} h:=\left(g_{2}^{\sigma}, g_{1}^{\sigma}\right)
$$

Write $\overline{\mathrm{GL}\left(V_{E}\right)}=\left\{h \in \mathrm{GL}\left(V_{E}\right) \times\left.\mathrm{GL}\left(V_{E}\right)\right|^{\sigma} h=h\right\}$. Then there exists an isomorphism of groups $\operatorname{GL}\left(V_{E}\right) \longrightarrow$ $\overline{\mathrm{GL}}\left(V_{E}\right) ; g \longmapsto\left(g, g^{\sigma}\right)$. If given $h \in \mathrm{GL}\left(V_{E}\right) \times \mathrm{GL}\left(V_{E}\right), w \in \mathbb{W}=V_{E} \otimes_{E} V_{E}$, one can verify that ${ }^{\sigma} h \cdot{ }^{\sigma} w={ }^{\sigma}(h \cdot w)$. So it induces a morphism from $\mathrm{GL}\left(V_{E}\right) \simeq \overline{\mathrm{GL}\left(V_{E}\right)}$ to $\mathrm{GO}(M)$. By the fixed basis $\left\{e_{1}, e_{2}\right\}$, we obtain a morphism $i: H=\mathrm{GL}_{2}(E) \longrightarrow \mathrm{GO}(M)$.

Lemma 3.1. The morphism $i: H=\mathrm{GL}_{2}(E) \longrightarrow \mathrm{GO}(M)$ is defined by $H \times M \longrightarrow M ;(h, m) \longmapsto h m \bar{h}^{t}$, where $\bar{h}^{t}$ is the transpose conjugate of $h$.

Proof. Let $h=\left(\begin{array}{ll}a & b \\ c & d\end{array}\right) \in H$. By definition, $h \cdot\left(e_{1}, e_{2}\right):=\left(e_{1}, e_{2}\right)\left(\begin{array}{ll}a & b \\ c & d\end{array}\right)=\left(a e_{1}+c e_{2} ; b e_{1}+d e_{2}\right)$. So $(h, 1) \cdot\left(\alpha e_{1} \otimes\right.$ $\left.e_{1}+\beta e_{1} \otimes e_{2}+\gamma e_{2} \otimes e_{1}+\delta e_{2} \otimes e_{2}\right)=(a \alpha+b \gamma) e_{1} \otimes e_{1}+(a \beta+b \delta) e_{1} \otimes e_{2}+(c \alpha+d \gamma) e_{2} \otimes e_{1}+(c \beta+d \delta) e_{2} \otimes e_{2}$. Forgetting 
the basis we obtain $(h, 1) \cdot\left(\begin{array}{ll}\alpha & \beta \\ \gamma & \delta\end{array}\right)=\left(\begin{array}{ll}a & b \\ c & d\end{array}\right)\left(\begin{array}{ll}\alpha & \beta \\ \gamma & \delta\end{array}\right)$. Similarly, $(1, \bar{h}) \cdot\left(\alpha e_{1} \otimes e_{1}+\beta e_{1} \otimes e_{2}+\gamma e_{2} \otimes e_{1}+\delta e_{2} \otimes e_{2}\right)$ $=(\alpha \bar{a}+\beta \bar{b}) e_{1} \otimes e_{1}+(\beta \bar{d}+\alpha \bar{c}) e_{1} \otimes e_{2}+(\gamma \bar{a}+\delta \bar{b}) e_{2} \otimes e_{1}+(\delta \bar{d}+\gamma \bar{c}) e_{2} \otimes e_{2}$, i.e. $(1, \bar{h}) \cdot\left(\begin{array}{ll}\alpha & \beta \\ \gamma & \delta\end{array}\right)=\left(\begin{array}{ll}\alpha \bar{a}+\beta \bar{b} & \beta \bar{d}+\alpha \bar{c} \\ \gamma \bar{a}+\delta \bar{b} & \delta \bar{d}+\gamma \bar{c}\end{array}\right)=$ $\left(\begin{array}{ll}\alpha & \beta \\ \gamma & \delta\end{array}\right)\left(\begin{array}{ll}\bar{a} & \bar{c} \\ \bar{b} & \bar{d}\end{array}\right)=\left(\begin{array}{ll}\alpha & \beta \\ \gamma & \delta\end{array}\right) \bar{h}^{t}$

Now we consider the symplectic vector space $V \otimes M$ over $F$ of dimension 8 . By the above discussion, there is a map from $G \times H$ to $\operatorname{GSp}\left(V \otimes_{F} M\right) \simeq \mathrm{GSp}_{8}(F)$. Similarly as in Section 2, we consider the restriction of the Weil representation $\left(\rho, \operatorname{GSp}_{8}(F), W\right)$ to the group $G \times H$, denoted by $(\pi, G \times H, W)$.

Proposition 3.2. The Weil representation $(\pi, G \times H, W)$ can be realized in the space $W=\mathbb{C}\left[M \times X_{F}\right]$, and the action of $G \times H$ on $W$ is given by the following formulas:

$$
\begin{gathered}
(\pi([h(a), 1]) F)(m, \psi)=F(a m, \psi), \\
(\pi([u(b), 1]) F)(m, \psi)=\psi(b \operatorname{det}(m)) F(m, \psi), \\
(\pi([\omega, 1]) F)(m, \psi)=-q^{-2} \sum_{n \in M} F(n, \psi) \psi(B(m, n)), \\
\left(\pi\left(\left[h^{\prime}(t), 1\right]\right) F\right)(m, \psi)=F\left(m, \psi^{t^{-1}}\right), \\
(\pi([1, h]) F)(m, \psi)=F\left(h^{-1} m h^{\star-1} \mathrm{~N}_{E / F}(\operatorname{det}(h)), \psi^{\mathrm{N}_{E / F}\left(\operatorname{det}(h)^{-1}\right)}\right),
\end{gathered}
$$

where $h(a), u(b), h^{\prime}(t) \in G ; h \in H, m \in M, \psi \in X_{F} ; B(m, n):=q(m+n)-q(m)-q(n)$ for $m, n \in M$.

Proof. (3.1), (3.2) and (3.4) follow directly from (1.1) - (1.4) in Section 1 . For (3.5):

$$
\begin{gathered}
(\pi([1, h]) F)(m, \psi)=\rho\left(\left(\begin{array}{ll}
h & 0 \\
0 & h
\end{array}\right)\right) F(m, \psi)=\rho\left(\left(\begin{array}{cc}
1 & 0 \\
0 & \mathrm{~N}_{E / F}(\operatorname{det}(h))
\end{array}\right)\right) \rho\left(\left(\begin{array}{ll}
h & 0 \\
0 & h
\end{array}\right) \cdot\left(\begin{array}{cc}
1 & 0 \\
0 & \mathrm{~N}_{E / F}(\operatorname{det}(h))^{-1}
\end{array}\right)\right) F(m, \psi) \\
=F\left(h^{-1} \cdot \mathrm{N}_{E / F}(\operatorname{det}(h)) m, \psi^{\mathrm{N}_{E / F}(\operatorname{det}(h))^{-1}}\right)=F\left(h^{-1} m h^{\star-1} \mathrm{~N}_{E / F}(\operatorname{det}(h)), \psi^{\mathrm{N}_{E / F}\left(\operatorname{det}(h)^{-1}\right)}\right) .
\end{gathered}
$$

For (3.3): Let $E=F(\xi)$ with $\operatorname{Tr}(\xi)=0$. Take $f_{1}=\left(\begin{array}{cc}1 & 0 \\ 0 & \frac{1}{2}\end{array}\right), f_{2}=\left(\begin{array}{cc}1 & 0 \\ 0 & -\frac{1}{2}\end{array}\right), f_{3}=\left(\begin{array}{ll}0 & 1 \\ 1 & 0\end{array}\right), f_{4}=\left(\begin{array}{ll}0 & \xi \\ \bar{\xi} & 0\end{array}\right)$. Suppose $\mathrm{N}_{E / F}(\xi)=\bar{\xi} \xi=-\xi^{2}=a$. Then the set $\left\{e_{1} \otimes f_{1}, e_{1} \otimes f_{2}, e_{1} \otimes f_{3}, e_{1} \otimes f_{4} ; e_{2} \otimes f_{1},-e_{2} \otimes f_{2},-\frac{1}{2} e_{2} \otimes f_{3},-\frac{1}{2} a^{-1} e_{2} \otimes f_{4}\right\}$ is a symplectic basis of $V \otimes_{F} M$. By such basis, the image of $\left(\begin{array}{cc}0 & 1 \\ -1 & 0\end{array}\right) \otimes 1$ in $\operatorname{GSp}\left(V \otimes_{F} M\right)$, under the map $\mathrm{GL}(V) \times \mathrm{GO}(M) \longrightarrow \mathrm{GSp}\left(V \otimes_{F} M\right)$, is $\bar{\omega}\left(\begin{array}{cc}A & 0 \\ 0 & A^{-1}\end{array}\right)$, where $\bar{\omega}=\left(\begin{array}{cc}0 & I \\ -I & 0\end{array}\right)$ and $A=\left(\begin{array}{cccc}1 & 0 & 0 & 0 \\ 0 & -1 & 0 & 0 \\ 0 & 0 & -2 & 0 \\ 0 & 0 & 0 & -2 a\end{array}\right)$. Applying the formulas (1.1), (1.3) in Section 1, we get

$$
\begin{gathered}
\rho\left(\bar{\omega}\left(\begin{array}{cc}
A & 0 \\
0 & A^{-1}
\end{array}\right)\right) F\left(e_{2} \otimes m, \psi\right)=q^{-2} \sum_{n \in M}\left(\rho\left(\begin{array}{cc}
A & 0 \\
0 & A^{-1}
\end{array}\right) F\right)\left(e_{2} \otimes n, \psi\right) \psi\left(\left\langle e_{2} \otimes n, \bar{\omega}^{-1}\left(e_{2} \otimes m\right)\right\rangle\right) \\
=q^{-2} \chi_{q}^{+}\left(2^{2} \xi^{2}\right) \sum_{n \in M} F\left(\left(\begin{array}{cc}
A^{-1} & 0 \\
0 & A
\end{array}\right) \cdot e_{2} \otimes n, \psi\right) \psi\left(\left\langle e_{2} \otimes n, \bar{\omega}^{-1}\left(e_{2} \otimes m\right)\right\rangle\right)=-q^{-2} \sum_{n \in M} F\left(e_{2} \otimes n, \psi\right) \psi\left(\left\langle\left(\begin{array}{cc}
A & 0 \\
0 & A^{-1}
\end{array}\right) e_{2} \otimes n, \bar{\omega}^{-1}\left(e_{2} \otimes m\right)\right\rangle\right) \\
=-q^{-2} \sum_{n \in M} F\left(e_{2} \otimes n, \psi\right) \psi\left(\left\langle e_{2} \otimes n,\left(\bar{\omega}\left(\begin{array}{cc}
A & 0 \\
0 & A^{-1}
\end{array}\right)\right)^{-1}\left(e_{2} \otimes m\right)\right\rangle\right)=-q^{-2} \sum_{n \in M} F\left(e_{2} \otimes n, \psi\right) \psi\left(\left\langle e_{2} \otimes n,\left(\begin{array}{cc}
0 & 1 \\
-1 & 0
\end{array}\right)^{-1}\left(e_{2}\right) \otimes m\right\rangle\right) \\
=-q^{-2} \sum_{n \in M} F\left(e_{2} \otimes n, \psi\right) \psi\left(\left\langle e_{2} \otimes n,-e_{1} \otimes m\right\rangle\right)=-q^{-2} \sum_{n \in M} F\left(e_{2} \otimes n, \psi\right) \psi(B(m, n)) .
\end{gathered}
$$

Forgetting the basis, we get the formula 3.3 .

Remark 3.3. The above representation $\pi$ of $G \times H$ is compatible with $\rho_{Q}$ constructed by Andrade in [1, p.35, Theorem 5] (There the $\rho_{Q}$ is defined more directly). 
3.2. To decompose the representation $\pi$ of $G \times H$, let us calculate the dimension of the vector space $W\left[\pi_{1}\right]$ ( see below for its definition).

Let $U=\left\{x \in E^{\times} \mid N_{E / F}(x)=1\right\}$. We regard $U$ as a subgroup of $H$. Let $\operatorname{Irr}_{0}(H)$ be the set of the isomorphism classes of the irreducible representations $\pi_{1}$ of $H$, such that $\pi_{1}$ is trivial over $U$. For each representation $\left(\pi_{1}, V_{1}\right) \in$ $\operatorname{Irr}_{0}(H)$, we associate a representation $\left(\pi_{0}, W\left[\pi_{1}\right]\right)$ of $G$, where the vector space $W\left[\pi_{1}\right]$ consists of functions: $f$ : $M \times X_{F} \longrightarrow V_{1}$ such that

$$
f\left(h m h^{\star} \mathrm{N}_{E / F}\left(\operatorname{det}(h)^{-1}\right), \psi^{\mathrm{N}_{E / F}(\operatorname{det}(h))}\right)=\pi_{1}(h) \circ f(m, \psi)
$$

for $h \in H,(m, \psi) \in M \times X_{F}$ and the action of $G$ on $W\left[\pi_{1}\right]$ is given by the formulas 3.1 - 3.4).

Proposition 3.4. For the representation $(\pi, G \times H, W)$, we have the following decomposition:

$$
\pi \simeq \bigoplus_{\left(\pi_{1}, V_{1}\right) \in \operatorname{Irr}_{0}(H)} W\left[\pi_{1}\right] \otimes \check{V}_{1}
$$

Proof. The representation $W$ has the decomposition $\pi=\oplus_{\left(\pi_{1}, V_{1}\right)} W_{\check{\pi}_{1}} \otimes \check{V}_{1}$ and then $W_{\check{\pi}_{1}} \simeq\left(W \otimes V_{1}\right)^{H} \simeq W\left[\pi_{1}\right]$. The action of $G$ on $W\left[\pi_{1}\right]$ arises from the definition of $\pi$ and above isomorphisms.

For $\left(\pi_{1}, V_{1}\right) \in \operatorname{Irr}_{0}(H)$, we define $W\left[\pi_{1}\right](\xi)=\left\{f(\xi) \mid f \in W\left[\pi_{1}\right]\right\}$ and an $H$-action on the set $M \times X_{F}$ as follows:

$$
h \cdot(m, \psi):=\left(h m h^{\star} \mathrm{N}_{E / F}(\operatorname{det}(h))^{-1}, \psi^{\mathrm{N}_{E / F}(\operatorname{det}(h))}\right) \quad h \in H, \psi \in X_{F}, m \in M .
$$

It is observed that $W\left[\pi_{1}\right](\xi)=V_{1}^{\mathrm{Stab}_{H}(\xi)}$ for any $\xi \in M \times X_{F}$.

Proposition 3.5. Consider the action of $\mathrm{H}$ on $\mathrm{M} \times X_{F}$.

(1) The distinct orbit of this action can be described as follows:

(i) Orbit $\left\{\xi_{a}\right\}$, where $\xi_{a}=\left(\left(\begin{array}{ll}1 & 0 \\ 0 & 1\end{array}\right), \phi^{a}\right) \quad$ for any $a \in F^{\times}$;

(ii) Orbit $\{\eta\}$, where $\eta=\left(\left(\begin{array}{ll}1 & 0 \\ 0 & 0\end{array}\right), \phi\right)$;

(iii) Orbit $\{\delta\}$, where $\delta=\left(\left(\begin{array}{ll}0 & 0 \\ 0 & 0\end{array}\right), \phi\right)$.

(2) The corresponding stabilizer of the canonical element in each orbit is presented as following:

(i) $\operatorname{Stab}_{H}\left(\xi_{a}\right)=U_{2}(E)$, where $U_{2}(E)=\left\{h \in H \mid h h^{\star}=1\right\}$;

(ii) $\operatorname{Stab}_{H}(\eta)=H_{1}$, where $H_{1}=\left\{h=\left(\begin{array}{cc}u & b \\ 0 & v\end{array}\right) \mid u, v \in U, b \in E\right\}$;

(iii) $\operatorname{Stab}_{H}(\delta)=H_{2}$, where $H_{2}=\{h \in H \mid \operatorname{det}(h) \in U\}$.

Proof. We transfer the $H$-action $\cdot$ to another $H$-action $\odot$, where $\odot$ is defined by $h \odot(m, \psi):=\left(h m h^{\star}, \psi^{\mathrm{N}_{E / F}\left(\operatorname{det}(h)^{-1}\right)}\right)$. Since $\alpha: H \longrightarrow H ; h \longmapsto h / \operatorname{det}(h)$ is a group isomorphism and $\alpha(h) \odot(m, \psi)=h \cdot(m, \psi)$, it reduces to consider the action $\odot$.

1) Every element $m \in M$ corresponds to a hermitian form on a 2-dimension $E$-vector space $V$. By the property of hermitian form over finite fields and the surjection of the morphism $\mathrm{N}_{E / F}: E^{\times} \longrightarrow F^{\times}$, one can find $h \in H$ such that $h m h^{\star}=\operatorname{diag}(a, b)$ where $(a, b)=(1,1),(1,0)$ or $(0,0)$. Let $H_{a, b}=\operatorname{Stab}_{H}(\operatorname{diag}(a, b))$. By calculation, we know: (a) $H_{1,1}=\left\{h \in H \mid h h^{\star}=1\right\}$; (b) $H_{1,0}=\left\{\left(\begin{array}{ll}u & b \\ 0 & v\end{array}\right) \in H \mid u \in U\right\}$; (c) $H_{0,0}=H$. Consequently we define an action of $H_{a, b}$ on $X_{F}$ by $(h, \psi) \longrightarrow \psi^{\mathrm{N}_{E / F}(\operatorname{det}(h))}$ for $h \in H_{a, b}$ and $\psi \in X_{F}$. As above, the results follow by determining the orbits.

2) It is straightforward.

Lemma 3.6.

$$
\text { (i) } U_{2}(E)=\left\{\left(\begin{array}{cc}
u & 0 \\
0 & 1
\end{array}\right)\left(\begin{array}{cc}
a & b \\
-b^{q} & a^{q}
\end{array}\right) \mid u \in U, a, b \in E, \mathrm{~N}_{E / F}(a)+\mathrm{N}_{E / F}(b)=1\right\} \text {; }
$$

(ii) $\left|U_{2}(E)\right|=(q-1) q(q+1)^{2}$;

(iii) If we choose an element $e_{-1} \in E^{\times}$, such that $e_{-1}^{q+1}=-1$, then the element $\left(\begin{array}{cc}0 & 1 \\ -1 & e_{-1}^{q}\end{array}\right) \notin B^{\prime} U_{2}(E)$; 
(iv) $H=B^{\prime} U_{2}(E) \cup B^{\prime}\left(\begin{array}{cc}0 & 1 \\ -1 & e_{-1}^{q}\end{array}\right) U_{2}(E)$.

Proof. (i)(ii) follow from [1, p. 242-243].

iii)

$$
\begin{aligned}
B^{\prime} U_{2}(E) & =B^{\prime} \mathrm{SU}_{2}(E)=\left\{\left(\begin{array}{cc}
x & y \\
0 & z
\end{array}\right) \cdot\left(\begin{array}{cc}
a & b \\
-b^{q} & a^{q}
\end{array}\right) \mid x, z \in E^{\times} ; a, b, y \in E ; \mathrm{N}_{E / F}(a)+\mathrm{N}_{E / F}(b)=1\right\} \\
& =\left\{\left(\begin{array}{cc}
x a-y b^{q} & x b+y a^{q} \\
-z b^{q} & z a^{q}
\end{array}\right) \mid x, z \in E^{\times} ; a, b, y \in E ; \mathrm{N}_{E / F}(a)+\mathrm{N}_{E / F}(b)=1\right\} .
\end{aligned}
$$

So, $\mathrm{N}_{E / F}\left(-b^{q} z\right)+\mathrm{N}_{E / F}\left(a^{q} z\right)=\mathrm{N}_{E / F}(z) \neq 0$. On the other hand, $\mathrm{N}_{E / F}(-1)+\mathrm{N}_{E / F}\left(-e_{-1}^{q}\right)=0$; this implies the result. iv) It is enough to check that they have the same cardinality, i.e.

By calculation,

$$
|H|=\left|B^{\prime} U_{2}(E)\right|+\left|B^{\prime}\left(\begin{array}{cc}
0 & 1 \\
-1 & e_{-1}^{q}
\end{array}\right) U_{2}(E)\right|
$$

$$
B^{\prime} \cap U_{2}(E)=\left\{\left(\begin{array}{cc}
u & 0 \\
0 & 1
\end{array}\right)\left(\begin{array}{cc}
a & 0 \\
0 & a^{q}
\end{array}\right) \mid u, a \in U\right\} .
$$

So

$$
\left|B^{\prime} U_{2}(E)\right|=\left|B^{\prime}\right|\left|\frac{U_{2}(E)}{B^{\prime} \cap U_{2}(E)}\right|=\left|B^{\prime}\right| \cdot(q-1) q .
$$

Now let

Then

$$
g=\left(\begin{array}{cc}
u a & u b \\
-b^{q} & a^{q}
\end{array}\right) \in U_{2}(E), g_{0}=\left(\begin{array}{cc}
0 & 1 \\
-1 & e_{-1}^{q}
\end{array}\right)
$$

$$
g_{0} g g_{0}^{-1}=\left(\begin{array}{cc}
\left(a-b e_{-1}\right)^{q} & b^{q} \\
e_{-1}^{q}\left[\left(a-e_{-1} b\right)^{q}-u\left(a-e_{-1} b\right)\right] & u a+\left(b e_{-1}\right)^{q}
\end{array}\right),
$$

which is an element of $B^{\prime}$ if and only if $\left(a-b e_{-1}\right)^{q-1}=u$. Since $\operatorname{det}\left(g_{0} g g_{0}^{-1}\right)=u$, we have

$$
B^{\prime} \cap g_{0} U_{2}(E) g_{0}^{-1}=\left\{\left(\begin{array}{cc}
\left(a-b e_{-1}\right)^{q} & b^{q} \\
0 & \left(a-b e_{-1}\right)^{-1}
\end{array}\right) \mid \mathrm{N}_{E / F}(a)+\mathrm{N}_{E / F}(b)=1\right\} .
$$

So

From this, we obtain

$$
\left|B^{\prime} \cap g_{0} U_{2}(E) g_{0}^{-1}\right|=\left|\mathrm{SU}_{2}(E)\right|=(q-1) q(q+1)
$$

and

Finally

$$
\left|g_{0} U_{2}(E) g_{0}^{-1} / B^{\prime} \cap g_{0} U_{2}(E) g_{0}^{-1}\right|=q+1
$$

$$
\left|B^{\prime} g_{0} U_{2}(E)\right|=\left|B^{\prime}\right|\left|g_{0} U_{2}(E) g_{0}^{-1} / B^{\prime} \cap g_{0} U_{2}(E) g_{0}^{-1}\right|=\left|B^{\prime}\right| \cdot(q+1) \text {. }
$$

$$
\left|B^{\prime} U_{2}(E)\right|+\left|B^{\prime} g_{0} U_{2}(E)\right|=\left|B^{\prime}\right| \cdot(q-1) q+\left|B^{\prime}\right| \cdot(q+1)=\left|B^{\prime}\right| \cdot\left(q^{2}+1\right)=|H| .
$$

Let us determine the dimension of the vector space $W\left[\pi_{1}\right]$ for each $\pi_{1} \in \operatorname{Irr}_{0}(H)$.

Lemma 3.7. Let $\left(\pi_{1}, V_{1}\right)$ be an irreducible representation of $H$ in $\operatorname{Ir}_{0}(H)$. Then:

(1) In the case $\pi_{1}=\Psi \cdot 1_{H}$ with $\Psi \in \operatorname{Irr}\left(E^{\times}\right)$, we have

(a) $W\left[\pi_{1}\right]=0$, if $\Psi \neq \Psi^{q}$.

(b) $\operatorname{dim}_{\mathbb{C}} W\left[\pi_{1}\right]\left(\xi_{a}\right)=\operatorname{dim}_{\mathbb{C}} W\left[\pi_{1}\right](\eta)=\operatorname{dim}_{\mathbb{C}} W\left[\pi_{1}\right](\delta)=1$, if $\Psi=\Psi^{q}$.

(2) In the case $\pi_{1}=\Psi \cdot \mathrm{St}_{H}$ with $\Psi \in \operatorname{Irr}\left(E^{\times}\right)$, we have

(a) $W\left[\pi_{1}\right]=0$, if $\Psi \neq \Psi^{q}$.

(b) $\operatorname{dim}_{\mathbb{C}} W\left[\pi_{1}\right]\left(\xi_{a}\right)=\operatorname{dim}_{\mathbb{C}} W\left[\pi_{1}\right](\eta)=1$ and $\operatorname{dim}_{\mathbb{C}} W\left[\Psi \cdot \mathrm{St}_{H}\right](\delta)=0$, if $\Psi=\Psi^{q}$.

(3) In the case $\pi_{1}=\Pi_{\Lambda, \Sigma}$ with $\Lambda \neq \Sigma \in \operatorname{Irr}\left(E^{\times}\right)$, we have

(a) $\operatorname{dim}_{\mathbb{C}} W\left[\Pi_{\Lambda, \Sigma}\right]\left(\xi_{a}\right)=1, \operatorname{dim}_{\mathbb{C}} W\left[\Pi_{\Lambda, \Sigma}\right](\eta)=2, \operatorname{dim}_{\mathbb{C}} W\left[\Pi_{\Lambda, \Sigma}\right](\delta)=0$, if $\Lambda=\Lambda^{q}$ and $\Sigma=\Sigma^{q}$.

(b) $\operatorname{dim}_{\mathbb{C}} W\left[\Pi_{\Lambda, \Sigma}\right]\left(\xi_{a}\right)=1, \operatorname{dim}_{\mathbb{C}} W\left[\Pi_{\Lambda, \Sigma}\right](\eta)=\operatorname{dim}_{\mathbb{C}} W\left[\Pi_{\Lambda, \Sigma}\right](\delta)=0$, if $\Lambda=\Sigma^{q}$ and $\Sigma=\Lambda^{q}$. 
For the other kind of $\Lambda \neq \Sigma \in \operatorname{Irr}\left(E^{\times}\right), W\left[\Pi_{\Lambda, \Sigma}\right]=0$.

(4) In the case $\pi_{1}=\Pi_{\Theta}$, where $\Theta \in \operatorname{Irr}\left(E_{1}^{\times}\right)-\operatorname{Irr}\left(E^{\times}\right)$for some quadratic extension $E_{1}$ of $E$, we have

(a) $W\left[\Pi_{\Theta}\right]=0$.

Proof. 1) By Proposition 3.5 (2), we know that the image of the map det : $\operatorname{Stab}_{H}(\xi) \longrightarrow E^{\times}$is $U$ for $\xi=\xi_{a}, \eta, \delta$. So $V_{1}^{\mathrm{Stab}_{H}(\xi)}=\left\{\begin{array}{cl}V_{1} & \text { if } \Psi \text { is trivial over } U \\ 0 & \text { otherwise. }\end{array}\right.$

2) Let $\left(\pi_{2}, V_{2}\right)=\operatorname{Ind}_{B^{\prime}}^{H}\left(\Psi \cdot 1_{B^{\prime}}\right)$. By definition, $W\left[\pi_{2}\right](\xi)=W\left[\Psi \cdot 1_{H}\right](\xi) \oplus W\left[\Psi \cdot \mathrm{St}_{H}\right](\xi)$. The dimension of $W\left[\Psi \cdot 1_{H}\right](\xi)$ is known, so it remains to calculate the dimension $W\left[\pi_{2}\right](\xi)$ for $\xi=\xi_{a}, \eta, \delta$.

(a) $\xi=\xi_{a}$. In this case, $\operatorname{Stab}_{H}(\xi)=U_{2}(E)$ and $H=B^{\prime} U_{2}(E) \cup B^{\prime}\left(\begin{array}{cc}0 & 1 \\ -1 & e_{-1}^{q}\end{array}\right) U_{2}(E)$ for $e_{-1} \in E^{\times}$satisfying $\mathrm{N}_{E / F}\left(e_{-1}\right)=-1$. So $V_{2}^{U_{2}(E)}$ is generated by the following functions $\alpha, \beta$ :

1. The support of $\alpha$ is $B^{\prime} U_{2}(E), \alpha(b u)=\Psi \cdot 1_{B^{\prime}}(b)$ for $b \in B^{\prime}, u \in U_{2}(E)$, and $\Psi \cdot 1_{B^{\prime}}$ is trivial over $B^{\prime} \cap U_{2}(E)$.

2. The support of $\beta$ is $B^{\prime}\left(\begin{array}{cc}0 & 1 \\ -1 & e_{-1}^{q}\end{array}\right) U_{2}(E), \beta\left(b\left(\begin{array}{cc}0 & 1 \\ -1 & e_{-1}^{q}\end{array}\right) u\right)=\Psi \cdot 1_{B^{\prime}}(b)$ and $\Psi \cdot 1_{B^{\prime}}$ is trivial over $B^{\prime} \cap$ $\left(\begin{array}{cc}0 & 1 \\ -1 & e_{-1}^{q}\end{array}\right) U_{2}(E)\left(\begin{array}{cc}0 & 1 \\ -1 & e_{-1}^{q}\end{array}\right)^{-1}$

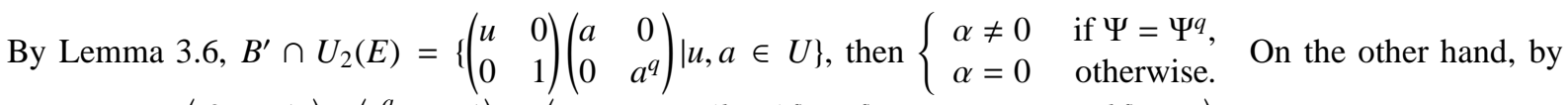
Lemma3.6 $\left(\begin{array}{cc}0 & 1 \\ -1 & e_{-1}^{q}\end{array}\right) g\left(\begin{array}{cc}e_{-1}^{q} & -1 \\ 1 & 0\end{array}\right)=\left(\begin{array}{cc}-\left(b e_{-1}\right)^{q}+a^{q} & b^{q} \\ u\left(-b-a e_{-1}^{q}\right)+\left(a e_{-1}\right)^{q}-\left(b e_{-1}^{2}\right)^{q} & u a+\left(b e_{-1}\right)^{q}\end{array}\right) \in B^{\prime}$, which implies that $u\left(a e_{-1}^{q}+b\right)=\left(a e_{-1}\right)^{q}-\left(b e_{-1}^{2}\right)^{q}, \mathrm{~N}_{E / F}(a)+\mathrm{N}_{E / F}(b)=1$ and $u \in U$. In particular, in case $a=0, u=\left(e_{-1} b\right)^{q-1}$ and $\mathrm{N}_{E / F}\left(e_{-1} b\right)=-1$; in case $b=0, u=a^{q-1}$ and $\mathrm{N}_{E / F}(a)=1$. By calculation, we see $U=\left\{u \mid u=a^{q-1}\right.$ with $a \in$ $E^{\times}$and $\left.\mathrm{N}_{E / F}(a)= \pm 1\right\}$. Hence $\left\{\operatorname{det}(g)=u \mid\left(\begin{array}{cc}0 & 1 \\ -1 & e_{-1}^{q}\end{array}\right) g\left(\begin{array}{cc}e_{-1}^{q} & -1 \\ 1 & 0\end{array}\right) \in B^{\prime}, g=\left(\begin{array}{ll}u & 0 \\ 0 & 1\end{array}\right)\left(\begin{array}{cc}a & b \\ -b^{q} & a^{q}\end{array}\right) \in U_{2}(E)\right\}=U$. Finally, we see $\begin{cases}\beta \neq 0 & \text { if } \Psi=\Psi^{q}, \\ \beta=0 & \text { otherwise. }\end{cases}$

(b) $\xi=\eta$. In this case, $\operatorname{Stab}_{H}(\xi)=H_{1}=N^{\prime} \rtimes T^{\prime \prime}$ and $V_{2}^{H_{1}}=\left(V_{2}^{N^{\prime}}\right)^{T^{\prime \prime}}$ for $T^{\prime \prime}=\left\{\left(\begin{array}{ll}u & 0 \\ 0 & v\end{array}\right) \mid u, v \in U\right\}$. By Lemma2.3, as shown above, the vector space $V_{2}^{N^{\prime}}$ is generated by the functions $f_{\Psi, \Psi}, g_{\Psi, \Psi}$. We know that $t \cdot f_{\Psi, \Psi}=\Psi \otimes \Psi(t) f_{\Psi, \Psi}$, and $t \cdot g_{\Psi, \Psi}=\Psi \otimes \Psi(t) g_{\Psi, \Psi}$ for $t \in T$. So $V_{2}^{H_{1}}=\left\{\begin{array}{cc}\left\{f_{\Psi, \Psi}, g_{\Psi, \Psi}\right\} & \text { if } \Psi=\Psi^{q} \text {, } \\ 0 & \text { otherwise. }\end{array}\right.$

(c) $\xi=\delta$. In this case, we have $\operatorname{Stab}_{H}(\delta)=H_{2}, H=B^{\prime} H_{2}$ and $B^{\prime} \cap H_{2}=\left\{\left(\begin{array}{ll}a & b \\ 0 & d\end{array}\right) \mid a d \in U\right\}$. So $\operatorname{dim}_{\mathbb{C}} V_{2}^{H_{2}}=$ $\left\{\begin{array}{rr}1 & \text { if }\left.\Psi\right|_{U}=i d_{U} \\ 0 & \text { otherwise. }\end{array}\right.$

3) (a) In case $\xi=\xi_{a}, V_{1}^{U_{2}(E)}$ is generated by the following two functions $\alpha, \beta$ in $V_{1}$ :

1. The support of $\alpha$ belongs to $B^{\prime} U_{2}(E), \alpha(b u):=\Lambda \Sigma(b)$ for $b \in B^{\prime}, u \in U_{2}(E)$ and $\Lambda \otimes \Sigma$ is trivial over $B^{\prime} \cap U_{2}(E)$

2. The support of $\beta$ belongs to $B^{\prime}\left(\begin{array}{cc}0 & 1 \\ -1 & e_{-1}^{q}\end{array}\right) U_{2}(E), \beta\left(b\left(\begin{array}{cc}0 & 1 \\ -1 & e_{-1}^{q}\end{array}\right) u\right):=\Lambda \otimes \Sigma(b)$ and $\Lambda \otimes \Sigma$ is trivial over $B^{\prime} \cap\left(\begin{array}{cc}0 & 1 \\ -1 & e_{-1}^{q}\end{array}\right) U_{2}(E)\left(\begin{array}{cc}e_{-1}^{q} & -1 \\ 1 & 0\end{array}\right)$

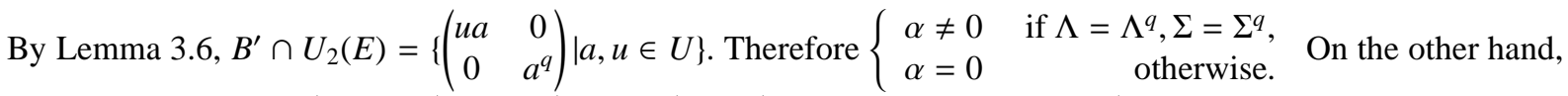
by Lemma 3.6, $B^{\prime} \cap\left(\begin{array}{cc}0 & 1 \\ -1 & e_{-1}^{q}\end{array}\right) U_{2}(E)\left(\begin{array}{cc}e_{-1}^{q} & -1 \\ 1 & 0\end{array}\right)=\left\{\left(\begin{array}{cc}\left(a-b e_{-1}\right)^{q} & b^{q} \\ 0 & \left(a-b e_{-1}\right)^{-1}\end{array}\right) \mid \mathrm{N}_{E / F}(a)+\mathrm{N}_{E / F}(b)=1\right\}$. Let $t=a-b e_{-1}, s=a+b e_{-1}$; then $\mathrm{N}_{E / F}(a)+\mathrm{N}_{E / F}(b)=1$ is equivalent to $t s^{q}+s t^{q}=2$. And $\beta \neq 0$ if and only if $\beta\left(\left(\begin{array}{cc}t^{q} & b^{q} \\ 0 & t^{-1}\end{array}\right)\right)=\Lambda^{q} \Sigma^{-1}(t)=1$ for $t \in E^{\times}$satisfying $t s^{q}+s t^{q}=2$. Considering $t=s^{-q}$, we know 
$\left\{\begin{array}{lr}\beta \neq 0 & \text { if } \Sigma=\Lambda^{q}, \Sigma \neq \Lambda \\ \beta=0 & \text { otherwise. }\end{array}\right.$

(b) In case $\xi=\eta, \operatorname{Stab}_{H}(\xi)=N^{\prime} \rtimes T^{\prime \prime}$ and $V_{1}^{N^{\prime}}$ is generated by the functions $f_{\Lambda, \Sigma}, g_{\Lambda, \Sigma}$ defined in Lemma 2.3 , Considering the $T^{\prime \prime}$-action on $V_{1}^{N^{\prime}}$, we know $V_{1}^{H_{1}}=\left\{\begin{array}{lr}\left\{f_{\Lambda, \Sigma}, g_{\Lambda, \Sigma}\right\} & \text { if } \Lambda=\Lambda^{q}, \Sigma=\Sigma^{q}, \\ 0 & \text { otherwise. }\end{array}\right.$

(c) In case $\xi=\delta, \operatorname{Stab}_{H}(\xi)=H_{2}$, and $H=B^{\prime} H_{2}, B^{\prime} \cap H_{2}=\left\{\left(\begin{array}{ll}a & b \\ 0 & d\end{array}\right) \mid a d \in U\right\} . V_{1}^{H_{2}}$ is generated by the function $f$, where $f(b h)=(\Lambda \otimes \Sigma)(b)$ for $b \in B^{\prime}, h \in H_{2}$ such that $\Lambda \otimes \Sigma$ is trivial over $B^{\prime} \cap H_{2}$; this implies $V_{1}^{H_{2}}=0$.

4) In case $\xi=\xi_{a}, U_{2}(E) \supseteq \mathrm{SU}_{2}(E)=\mathrm{SL}_{2}(E) \cap U_{2}(E)$, and there exists $h \in H$ such that $h \mathrm{SU}_{2}(E) h^{-1}=\mathrm{SL}_{2}(F)$ (see [1, p. 242, Proposition 4]). Hence $V_{1}^{U_{2}(E)} \subseteq V_{1}^{\mathrm{SU}_{2}(E)} \simeq V_{1}^{\mathrm{SL}_{2}(F)}$, which vanishes by [1, p. 82, Proposition 1]. If $\xi=\eta, \delta$, then $H_{i} \supseteq N^{\prime}$ and $V_{1}^{N^{\prime}}=0$. So $V_{1}^{H_{i}}=0$ for $i=1,2$.

Corollary 3.8. Let $\pi_{1}$ be an irreducible representation of $H$ in $\operatorname{Irr}_{0}(H)$. Then:

(i) $\operatorname{dim}_{\mathbb{C}} W\left[\pi_{1}\right]=q+1$, if $\pi_{1}=\Psi \cdot 1_{H}$ for $\Psi \in \operatorname{Irr}\left(E^{\times}\right)$with $\Psi=\Psi^{q}$.

(ii) $\operatorname{dim}_{\mathbb{C}} W\left[\pi_{1}\right]=q$, if $\pi_{1}=\Psi \cdot \operatorname{St}_{H}$ for $\Psi \in \operatorname{Irr}\left(E^{\times}\right)$with $\Psi=\Psi^{q}$.

(iii) $\operatorname{dim}_{\mathbb{C}} W\left[\pi_{1}\right]=q+1$, if $\pi_{1}=\Pi_{\Lambda, \Sigma}$ for $\Lambda \neq \Sigma \in \operatorname{Irr}\left(E^{\times}\right), \Lambda=\Lambda^{q}, \Sigma=\Sigma^{q}$.

(iv) $\operatorname{dim}_{\mathbb{C}} W\left[\pi_{1}\right]=q-1$, if $\pi_{1}=\Pi_{\Lambda, \Sigma}$ for $\Lambda \neq \Sigma \in \operatorname{Irr}\left(E^{\times}\right), \Lambda=\Sigma^{q}, \Sigma=\Lambda^{q}$.

And the above lists are all the representations $\pi_{1} \in \operatorname{Irr}_{0}(H)$, such that $W\left[\pi_{1}\right] \neq 0$.

Proof. As is known that $\operatorname{dim}_{\mathbb{C}} W\left[\pi_{1}\right]=\sum_{a \in F^{\times}} \operatorname{dim}_{\mathbb{C}} W\left[\pi_{1}\right]\left(\xi_{a}\right)+\operatorname{dim}_{\mathbb{C}} W\left[\pi_{1}\right](\eta)+\operatorname{dim}_{\mathbb{C}} W\left[\pi_{1}\right](\delta)$, so the results follow from above Lemma 3.7

3.3. The representation $\left(\pi_{0}, W\left[\pi_{1}\right]\right)$ I. In this subsection, let $\pi_{1}=\Psi \cdot 1_{H}$, where $\Psi=\Psi^{q} \in \operatorname{Irr}\left(E^{\times}\right)$and $\Psi=\psi \circ \mathrm{N}_{E / F}$ for some $\psi \in \operatorname{Irr}\left(F^{\times}\right)$. The vector space $W\left[\pi_{1}\right]$ is generated by the functions $F_{a}, R, S: M \times X_{F} \longrightarrow V_{1}$ for any $a \in F^{\times}$. Namely they all satisfy the equality (3.6) and

1. $\operatorname{supp} F_{a}=\operatorname{Orbit}\left\{\xi_{a}\right\}, F_{a}\left(\xi_{a}\right)=v_{0} \in V_{1}^{U_{2}(E)}$ for any $a \in F^{\times}$,

2. $\operatorname{supp} R=\operatorname{Orbit}\{\eta\}, R(\eta)=v_{1} \in V_{1}^{H_{1}}$,

3. $\operatorname{supp} S=\operatorname{Orbit}\{\delta\}, S(\delta)=v_{2} \in V_{1}^{H_{2}}$.

Lemma 3.9. For $t_{1}, t_{2}, r, r_{1} \neq r_{2} \in F^{\times}$, we have

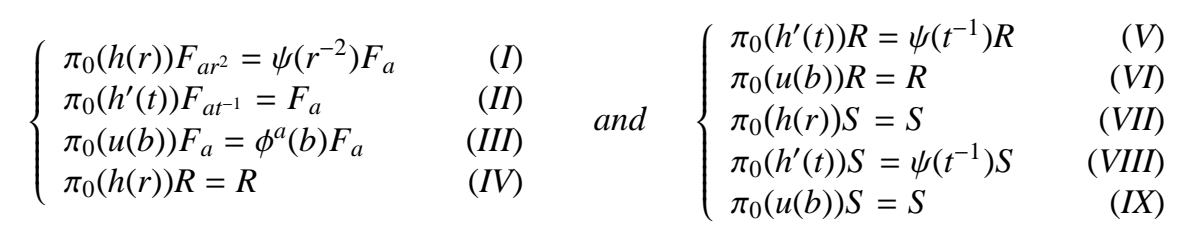

Proof. Firstly we know that $\operatorname{supp}\left(\pi_{0}(h(r)) F_{a r^{2}}\right) \subseteq\left\{(g, \psi) \mid g \in M, \operatorname{det}(g) \neq 0, \psi \in X_{F}\right\}$. Fix $i \in E^{\times}$such that $\mathrm{N}_{E / F}(i)=r$. Then

$$
\begin{aligned}
& \pi_{0}(h(r)) F_{a r^{2}}\left(\xi_{b}\right)=F_{a r^{2}}\left(\left(\begin{array}{cc}
r & 0 \\
0 & r
\end{array}\right), \phi^{b}\right)=F_{a r^{2}}\left(\mathrm{~N}_{E / F}(r)\left(\begin{array}{cc}
i^{-1} & 0 \\
0 & i^{-1}
\end{array}\right)\left(\begin{array}{ll}
1 & 0 \\
0 & 1
\end{array}\right)\left(\begin{array}{cc}
i^{-q} & 0 \\
0 & i^{-q}
\end{array}\right), \phi^{b}\right)
\end{aligned}
$$

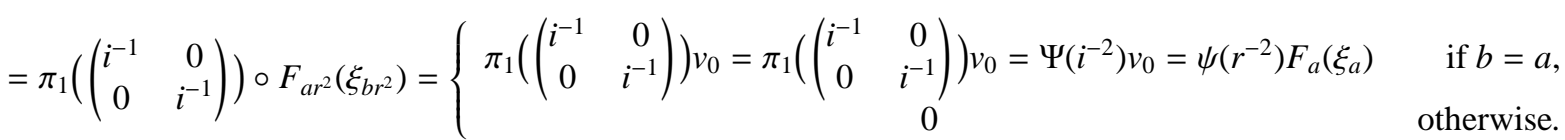

Hence (I) follows. Similarly, $\pi_{0}\left(h^{\prime}(t)\right) F_{a t^{-1}}\left(\xi_{a}\right)=F_{a t^{-1}}\left(\xi_{a t^{-1}}\right)=v_{0}=F_{a}\left(\xi_{a}\right)$. So we obtain (II), and (III) is clear. For (IV), by (3.1) - (3.4), we have $\pi_{0}(h(r)) R(\eta)=R\left(\left(\begin{array}{cc}r & 0 \\ 0 & 0\end{array}\right), \phi\right)=R\left(\left(\begin{array}{cc}i & 0 \\ 0 & i^{-1}\end{array}\right)\left(\begin{array}{cc}1 & 0 \\ 0 & 0\end{array}\right)\left(\begin{array}{cc}i^{q} & 0 \\ 0 & i^{-q}\end{array}\right), \phi^{a}\right)=$ $\pi_{1}\left(\left(\begin{array}{cc}i & 0 \\ 0 & i^{-1}\end{array}\right)\right) \circ R(\eta)=\pi_{1}\left(\left(\begin{array}{cc}i & 0 \\ 0 & i^{-1}\end{array}\right)\right) v_{1}=v_{1}=R(\eta)$, which implies our (IV). Now we let $x \in E^{\times}$satisfying $\mathrm{N}_{E / F}(x)=t$. Then

$$
\pi_{0}\left(h^{\prime}(t)\right) R(\eta)=R\left(\left(\begin{array}{ll}
1 & 0 \\
0 & 0
\end{array}\right), \phi^{t^{-1}}\right)=R\left(\mathrm{~N}_{E / F}(x)\left(\begin{array}{cc}
x^{-1} & 0 \\
0 & 1
\end{array}\right)\left(\begin{array}{cc}
1 & 0 \\
0 & 0
\end{array}\right)\left(\begin{array}{cc}
x^{-q} & 0 \\
0 & 1
\end{array}\right), \phi^{\mathrm{N}_{E / F}\left(x^{-1}\right)}\right)=\Psi\left(x^{-1}\right) R(\eta)=\psi\left(t^{-1}\right) R(\eta)
$$


thus we obtain (V). The following (VI), (VII) and (IX) are easy to verify. However

$$
\left(\pi_{0}\left(h^{\prime}(t)\right) S\right)(\delta)=S\left(\left(\begin{array}{ll}
0 & 0 \\
0 & 0
\end{array}\right), \phi^{t^{-1}}\right)=S\left(\mathrm{~N}_{E / F}(x)\left(\begin{array}{cc}
x^{-1} & 0 \\
0 & 1
\end{array}\right)\left(\begin{array}{cc}
0 & 0 \\
0 & 0
\end{array}\right)\left(\begin{array}{cc}
x^{-q} & 0 \\
0 & 1
\end{array}\right), \phi^{\mathrm{N}_{E / F}\left(x^{-1}\right)}\right)=\Psi\left(x^{-1}\right) S(\delta)=\psi\left(t^{-1}\right) S(\delta)
$$

in this way we verify (VIII).

Corollary 3.10. For $t_{1}, t_{2}, r, r_{1} \neq r_{2} \in F^{\times}$, we have
(1) $\operatorname{Tr} \pi_{0}\left(\begin{array}{ll}r & 0 \\ 0 & r\end{array}\right)=(q+1) \psi\left(r^{-2}\right)$.
(2) $\operatorname{Tr} \pi_{0}\left(\begin{array}{cc}r_{1} & 0 \\ 0 & r_{2}\end{array}\right)=2 \psi\left(r_{1}^{-1} r_{2}^{-1}\right)$.
(3) $\operatorname{Tr} \pi_{0}\left(\begin{array}{ll}r & 1 \\ 0 & r\end{array}\right)=\psi\left(r^{-2}\right)$.

Proposition 3.11. $\check{\pi}_{0} \simeq \psi \cdot \operatorname{Ind}_{B}^{G} 1_{G}$.

Proof. By Corollary 3.10, we know $\operatorname{Res}_{B}^{G} \pi_{0} \simeq\left(2 \sigma_{\psi^{-1}, \psi^{-1}}\right) \oplus\left(\psi^{-2} \otimes \sigma\right) \simeq \operatorname{Res}_{B}^{G}\left(\psi^{-1} \cdot \operatorname{Ind}_{B}^{G} 1_{B}\right)$ for irreducible representations $\sigma_{\psi^{-1}, \psi^{-1}}, \psi^{-1} \otimes \sigma$ of $B$ defined in Theorem 1.3 and the isotypic components $2 \sigma_{\psi^{-1}, \psi^{-1}}$ are spanned by the functions $R, S$. By Proposition 1.4, we have (i) $\pi_{0} \simeq \psi^{-1} \cdot \operatorname{Ind}_{B}^{G} 1_{G}$, or (ii) $\pi_{0} \simeq\left(2 \psi^{-1} \cdot 1_{G}\right) \oplus \pi_{\theta}$ for certain regular character $\theta$ of $E^{\times}$. But $\left(\pi_{0}(\omega) S\right)(\delta)=q^{-2} \sum_{n \in M} S(n, \phi) \phi(B(0, n))=q^{-2} S(\delta)$ so that $\pi_{0}(\omega) S \neq S$; this means that $\pi_{0}$ has at most only one isotypic component $\psi^{-1} \cdot 1_{G}$. Therefore the above case (ii) is impossible.

3.4. The representation $\left(\pi_{0}, W\left[\pi_{1}\right]\right)$ II. In this subsection, let $\pi_{1}=\Psi \cdot \mathrm{St}_{H}$, where $\Psi=\Psi^{q} \in \operatorname{Irr}\left(E^{\times}\right)$, $\Psi=$ $\psi \circ \mathrm{N}_{E / F}$ for some $\psi \in \operatorname{Irr}\left(F^{\times}\right)$. The vector space $W\left[\pi_{1}\right]$ is generated by the functions: $F_{a}, R: M \times X_{F} \longrightarrow V_{1}$ for any $a \in F^{\times}$. They all satisfy the equality (3.6), and

1. $\operatorname{supp}\left(F_{a}\right)=\operatorname{Orbit}\left\{\xi_{a}\right\}, F_{a}\left(\xi_{a}\right)=v_{0} \in V_{1}^{U_{2}(E)}$ for any $a \in F^{\times}$,

2. $\operatorname{supp}(R)=\operatorname{Orbit}\{\eta\}, R(\eta)=v_{1}=q^{2} f_{\Psi, \Psi}-g_{\Psi, \Psi} \in V_{1}^{H_{1}}$ by Lemma2.3

Similarly as in Section 3.3, we obtain:

Lemma 3.12.

$$
\left\{\begin{array} { l c } 
{ \pi _ { 0 } ( h ( r ) ) F _ { a r ^ { 2 } } = \psi ( r ^ { - 2 } ) F _ { a } } & { ( X I ) , } \\
{ \pi _ { 0 } ( h ^ { \prime } ( t ) ) F _ { a t ^ { - 1 } } = F _ { a } } & { ( X I I ) , } \\
{ \pi _ { 0 } ( u ( b ) ) F _ { a } = \phi ^ { a } ( b ) F _ { a } } & { ( X I I I ) , }
\end{array} \quad \text { and } \quad \left\{\begin{array}{ll}
\pi_{0}(h(r)) R=R & (X I V), \\
\pi_{0}\left(h^{\prime}(t)\right) R=\psi\left(t^{-1}\right) R & (X V), \\
\pi_{0}(u(b)) R=R & (X V I) .
\end{array}\right.\right.
$$

Lemma 3.13. Let $M^{(1)}=\{n \in M \mid$ rank $n=1\}$. Then:

(a) $M^{(1)}=\left\{\left(\begin{array}{ll}s & 0 \\ 0 & 0\end{array}\right) \mid s \in F^{\times}\right\} \cup\left\{s\left(\begin{array}{cc}\mathrm{N}_{E / F}(b) & b \\ b^{q} & 1\end{array}\right) \mid s \in F^{\times}, b \in E\right\}$.

(b) $s\left(\begin{array}{cc}\mathrm{N}_{E / F}(b) & b \\ b^{q} & 1\end{array}\right)=u(b)\left(\begin{array}{ll}0 & 0 \\ 0 & s\end{array}\right) u(b)^{\star}$ for $s \in F^{\times}$.

Proof. See [1, p.246-247].

Now, let us consider

$$
\begin{aligned}
& \left(\pi_{0}(\omega) R\right)(\eta)=-q^{-2} \sum_{n \in M} R(n, \phi) \phi\left(B\left(\left(\begin{array}{ll}
1 & 0 \\
0 & 0
\end{array}\right), n\right)\right) \stackrel{\operatorname{supp} R=M^{(1)}}{=}-q^{-2} \sum_{n \in M^{(1)}} R(n, \phi) \phi\left(B\left(\left(\begin{array}{ll}
1 & 0 \\
0 & 0
\end{array}\right), n\right)\right) \\
& =-q^{-2} \sum_{s \in F^{\star}}\left[R\left(\left(\begin{array}{cc}
s & 0 \\
0 & 0
\end{array}\right), \phi\right) \phi\left(B\left(\left(\begin{array}{cc}
1 & 0 \\
0 & 0
\end{array}\right),\left(\begin{array}{cc}
s & 0 \\
0 & 0
\end{array}\right)\right)\right)+\sum_{b \in E} R\left(u(b)\left(\begin{array}{ll}
0 & 0 \\
0 & s
\end{array}\right) u(b)^{\star}, \phi\right) \phi\left(B\left(\left(\begin{array}{ll}
1 & 0 \\
0 & 0
\end{array}\right), u(b)\left(\begin{array}{ll}
0 & 0 \\
0 & s
\end{array}\right) u(b)^{\star}\right)\right)\right] \\
& =-q^{-2} \sum_{s \in F^{\times}}\left[\pi_{0}(h(s)) R(\eta)+\sum_{b \in E} \pi_{1}\left(u(b)\left(\begin{array}{cc}
0 & -1 \\
1 & 0
\end{array}\right)\right) R\left(\left(\begin{array}{cc}
s & 0 \\
0 & 0
\end{array}\right), \phi\right) \phi(s)\right]=-q^{-2} \sum_{s \in F^{\times}}\left[v_{1}+\sum_{b \in E} \pi_{1}\left(u(b)\left(\begin{array}{cc}
0 & -1 \\
1 & 0
\end{array}\right)\right) R\left(\left(\begin{array}{cc}
s & 0 \\
0 & 0
\end{array}\right), \phi\right) \phi(s)\right] \\
& =-q^{-2} \sum_{s \in F^{\times}} \pi_{1}(u(b))\left[v_{1}+\phi(s) \sum_{b \in E} \pi_{1}\left(\left(\begin{array}{cc}
0 & -1 \\
1 & 0
\end{array}\right)\right) v_{1}\right]=-q^{-2} \pi_{1}(u(b))\left[(q-1) v_{1}-q^{2} \pi_{1}\left(\left(\begin{array}{cc}
0 & -1 \\
1 & 0
\end{array}\right)\right) v_{1}\right] \neq R(\eta) .
\end{aligned}
$$

It follows that

$$
\pi_{0}(\omega) R \neq R
$$


Proposition 3.14. $\check{\pi}_{0} \simeq \psi \cdot \mathrm{St}_{G}$.

Proof. By the formulas (XI)-(XVI), we obtain $\operatorname{Res}_{B}^{G} \pi_{0}=\operatorname{Res}_{B}^{G}\left(\psi^{-1} \cdot \operatorname{St}_{G}\right)$. Consequently by the formula (XVII), $\pi_{0}$ has no $\psi^{-1} \cdot 1_{G}$ isotypic component. Comparing this with Proposition 1.4 gives the result.

3.5. The representation $\left(\pi_{0}, W\left[\pi_{1}\right]\right)$ III. In this subsection, let $\pi_{1}=\Pi_{\Lambda, \Sigma}$ for $\Lambda \neq \Sigma$ and $\Lambda=\lambda \circ \mathrm{N}_{E / F}, \Sigma=$ $\sigma \circ \mathrm{N}_{E / F} \in \operatorname{Irr}\left(E^{\times}\right)$. The vector space $V_{1}^{H_{1}}\left(=V_{1}^{N^{\prime}}\right)$ is generated by the two functions $f_{\Lambda, \Sigma}, g_{\Lambda, \Sigma}$ defined in Lemma 2.3 Let $\Delta: M \times X_{F} \longrightarrow V_{1}$ satisfying (3.6) and $\operatorname{supp}(\Delta)=\operatorname{Orbit}\{\eta\}, \Delta(\eta)=f_{\Lambda, \Sigma}$. Then

$$
\begin{aligned}
& \text { (i) } \quad\left(\pi_{0}(h(r)) \Delta\right)(\eta)=\Delta\left(\left(\begin{array}{cc}
r & 0 \\
0 & 0
\end{array}\right), \phi\right)=\Delta\left(\left(\begin{array}{cc}
x & 0 \\
0 & x^{-1}
\end{array}\right)\left(\begin{array}{cc}
1 & 0 \\
0 & 0
\end{array}\right)\left(\begin{array}{cc}
x^{q} & 0 \\
0 & x^{-q}
\end{array}\right), \phi\right)=\Pi_{\Lambda, \Sigma}\left(\left(\begin{array}{cc}
x & 0 \\
0 & x^{-1}
\end{array}\right)\right) \Delta(\eta) \\
& =\Pi_{\Lambda, \Sigma}\left(\left(\begin{array}{cc}
x & 0 \\
0 & x^{-1}
\end{array}\right)\right)\left[f_{\Lambda, \Sigma}\right]=\Lambda(x) \Sigma\left(x^{-1}\right) f_{\Lambda, \Sigma}=\lambda(r) \sigma\left(r^{-1}\right) f_{\Lambda, \Sigma} \text { for } \mathrm{N}_{E / F}(x)=r . \\
& \left(\pi_{0}\left(h^{\prime}(t)\right) \Delta\right)(\eta)=\Delta\left(\left(\begin{array}{ll}
1 & 0 \\
0 & 0
\end{array}\right), \phi^{t^{-1}}\right)=\Delta\left(\mathrm{N}_{E / F}(x)\left(\begin{array}{cc}
x^{-1} & 0 \\
0 & 1
\end{array}\right)\left(\begin{array}{cc}
1 & 0 \\
0 & 0
\end{array}\right)\left(\begin{array}{cc}
x^{-q} & 0 \\
0 & 1
\end{array}\right), \phi^{\mathrm{N}_{E / F}\left(x^{-1}\right)}\right) \\
& =\Pi_{\Lambda, \Sigma}\left(\left(\begin{array}{cc}
x^{-1} & 0 \\
0 & 1
\end{array}\right)\right)[\Delta(\eta)]=\Pi_{\Lambda, \Sigma}\left(\left(\begin{array}{cc}
x^{-1} & 0 \\
0 & 1
\end{array}\right)\right)\left(f_{\Lambda, \Sigma}\right)=\Lambda\left(x^{-1}\right) f_{\Lambda, \Sigma}=\lambda\left(t^{-1}\right) f_{\Lambda, \Sigma}=\lambda\left(t^{-1}\right) \Delta(\eta) \text { for } \mathrm{N}_{E / F}(x)=t \text {. }
\end{aligned}
$$

By the above (i) and (ii), we obtain that $\pi_{0}(h(r)) \Delta=\lambda(r) \sigma\left(r^{-1}\right) \Delta, \pi_{0}\left(h^{\prime}(t)\right) \Delta=\lambda\left(t^{-1}\right) \Delta$. In particular, $\pi_{0}\left(\left(\begin{array}{cc}t_{1} & 0 \\ 0 & t_{2}\end{array}\right)\right) \Delta=\lambda\left(t_{2}^{-1}\right) \sigma\left(t_{1}^{-1}\right) \Delta$. It follows that $\operatorname{Hom}_{G}\left(\pi_{0}, \operatorname{Ind}_{B}^{G}\left(\lambda^{-1} \otimes \sigma^{-1}\right)\right) \simeq \operatorname{Hom}_{G}\left(\pi_{0}, \operatorname{Ind}_{B}^{G}\left(\sigma^{-1} \otimes \lambda^{-1}\right)\right) \neq 0$. Since $\operatorname{dim}_{\mathbb{C}} \pi_{0}=q+1$, surely $\pi_{0} \simeq \pi_{\lambda^{-1}, \sigma^{-1}}$.

3.6. The representation $\left(\pi_{0}, W\left[\pi_{1}\right]\right)$ IV. In this subsection, let $\pi_{1}=\Pi_{\Lambda, \Sigma}$ where $\Lambda \neq \Sigma \in \operatorname{Irr}\left(E^{\times}\right)$and $\Lambda=\Sigma^{q}, \Sigma=$ $\Lambda^{q}$. We start with recalling some explicit models for certain representations(cf. [1]).

3.6.1. I. Model for $\Pi_{\Lambda, \Sigma}$. By [1, p.21, Definition 2 ], $\Pi_{\Lambda, \Sigma}$ can be realized in the vector space $V_{1}$ spanned by all the functions $v: E^{2} \times E^{\times} \longrightarrow \mathbb{C}$ such that

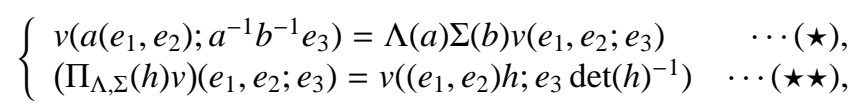

for $e_{1}, e_{2} \in E ; a, b, e_{3} \in E^{\times} ; h \in H$.

3.6.2. II. Model for $\pi_{\Lambda}$. Let $\pi_{\Lambda}$ be a cuspidal representation of $G$ corresponding to a regular character $\Lambda$ of $E^{\times}$. Invoking [1, p.53, Proposition 4], we know that $\pi_{\Lambda}$ can be realized in the vector space $\mathbb{C}\left[X_{F}\right]$ as follows:

$$
\begin{gathered}
\pi_{\Lambda}\left(\left(\begin{array}{ll}
r & 0 \\
0 & r
\end{array}\right)\right) f=\Lambda(r) f \\
\left(\pi_{\Lambda}\left(\left(\begin{array}{ll}
1 & 0 \\
0 & t
\end{array}\right)\right) f\right)(\psi)=f\left(\psi^{t^{-1}}\right) \\
\left(\pi_{\Lambda}\left(\left(\begin{array}{ll}
1 & s \\
0 & 1
\end{array}\right)\right) f\right)(\psi)=\psi(s) f(\psi) \\
\left(\pi_{\Lambda}(\omega) f\right)(\psi)=-q^{-1} \sum_{y \in E^{\times}} \psi\left(\operatorname{Tr}_{E / F}(y)\right) \Lambda(y) f\left(\psi^{N_{E / F}(y)}\right)
\end{gathered}
$$

where $\psi \in X_{F}, t, r \in F^{\times}, s \in F$. 
3.6.3. The representation $\left(\pi_{0}, W\left[\pi_{1}\right]\right)$. The vector space $W\left[\pi_{1}\right]$ is generated by those functions $F_{a}: M \times X_{F} \longrightarrow V_{1}$ satisfying (3.6) and $\operatorname{supp}\left(F_{a}\right)=\operatorname{Orbit}\left\{\xi_{a}\right\}, F_{a}\left(\xi_{a}\right)=v_{1} \in V_{1}^{U_{2}(E)}$ for any $a \in F^{\times}$. Using the above model, we choose an element $v_{1}$ as follows: $v_{1}: E^{2} \times E^{\times} \longrightarrow \mathbb{C}$ satisfies above $(\star)$ and

(1) $\operatorname{supp}\left(v_{1}\right)=\cup_{u \in U}$ Orbit $\left\{\left(1, u e_{-1} ; 1\right)\right\}$,

(2) $v_{1}\left(1, u e_{-1} ; 1\right)=\Lambda(u)$,

where Orbit $\left\{\left(1, u e_{-1} ; 1\right)\right\}=\left\{\left(a, u e_{-1} a ; a^{-1} b^{-1}\right) \in E^{2} \times E^{\times} \mid a, b \in E^{\times}\right\}$, and $e_{-1}$ is a fixed element in $E^{\times}$such that $\mathrm{N}_{E / F}\left(e_{-1}\right)=-1$.

Lemma 3.15. The above constructed $v_{1}$ belongs to $V_{1}^{U_{2}(E)}$.

Proof. 1) For $g=\left(\begin{array}{ll}u & 0 \\ 0 & 1\end{array}\right) \in \mathrm{U}_{2}(E)$, we have $\operatorname{supp}\left(g \cdot v_{1}\right)=\operatorname{supp}\left(v_{1}\right)$, and $g \cdot v_{1}\left(1, u_{0} e_{-1} ; 1\right)=v_{1}\left(u, u_{0} e_{1} ; u^{-1}\right)=$ $\Lambda(u) v_{1}\left(1, u_{0} u^{-1} e_{-1} ; 1\right)=\Lambda(u) \Lambda\left(u_{0} u^{-1}\right)=\Lambda\left(u_{0}\right)=v_{1}\left(1, u_{0} e_{-1} ; 1\right)$; thus $g \cdot v_{1}=v_{1}$.

2) For $g=\left(\begin{array}{cc}a & b \\ -b^{q} & a^{q}\end{array}\right) \in U_{2}(E)$, we have $\operatorname{supp}\left(g \cdot v_{1}\right)=\operatorname{supp}\left(v_{1}\right)$, and $g \cdot v_{1}\left(1, u e_{-1} ; 1\right)=v_{1}\left(a-b^{q} u e_{-1}, b+u e_{-1} a^{q} ; 1\right)=$ $v_{1}\left(a-b^{q} u e_{-1}, u e_{-1}\left(a-b^{q} u e_{-1}\right)^{q} ; 1\right)=\Lambda\left(a-b^{q} u e_{-1}\right) v_{1}\left(1, u e_{-1}\left(a-b^{q} u e_{-1}\right)^{q-1} ; a-b^{q} u e_{-1}\right)=\Lambda\left(a-b^{q} u e_{-1}\right) \Lambda(u(a-$ $\left.\left.b^{q} u e_{-1}\right)^{q-1}\right) \Sigma^{-1}\left(a-b^{q} u e_{-1}\right)=\Lambda(u)=v_{1}\left(1, u e_{-1} ; 1\right)$; therefore $g \cdot v_{1}=v_{1}$ in this case.

We define an intertwining operator between $\pi_{\Lambda^{-1}}$ and $\pi_{0}$ by

$$
j: \pi_{\Lambda^{-1}} \longrightarrow W\left[\pi_{1}\right] ; f \longmapsto j(f)=\sum_{a \in F^{\times}} f\left(\phi^{a}\right) F_{a} \text {, i.e. } j(f)\left(\xi_{a}\right)=f\left(\phi^{a}\right) v_{1} .
$$

Claim: $j\left(\pi_{\Lambda^{-1}}(g) f\right)=\pi_{0}(g) j(f)$ for $g \in G$.

Proof: (1) Let $g=\left(\begin{array}{ll}x & y \\ 0 & z\end{array}\right) \in B$.

$$
\begin{gathered}
\pi_{0}\left(\left(\begin{array}{ll}
x & y \\
0 & z
\end{array}\right)\right) j(f)\left(\xi_{a}\right)=\sum_{t \in F^{\times}} f\left(\phi^{t}\right) \pi_{0}\left(\left(\begin{array}{ll}
x & y \\
0 & z
\end{array}\right)\right) F_{t}\left(\xi_{a}\right) \\
=\sum_{t \in F^{\times}} f\left(\phi^{t}\right) \pi_{0}\left(\left(\begin{array}{cc}
x & 0 \\
0 & x^{-1}
\end{array}\right)\left(\begin{array}{cc}
1 & 0 \\
0 & x z
\end{array}\right)\left(\begin{array}{cc}
1 & x^{-1} y \\
0 & 1
\end{array}\right)\right) F_{t}\left(\xi_{a}\right) \\
=\sum_{t \in F^{\times}} f\left(\phi^{t}\right) F_{t}\left(\left(\begin{array}{cc}
x & 0 \\
0 & x
\end{array}\right), \phi^{a x^{-1} z^{-1}}\right) \phi^{a}\left(y z^{-1}\right) \\
\stackrel{\mathrm{N}_{E / F}(r)=x}{=} \sum_{t \in F^{\times}} f\left(\phi^{t}\right) F_{t}\left(\left(\begin{array}{cc}
r^{-1} & 0 \\
0 & r^{-1}
\end{array}\right)\left(\begin{array}{cc}
1 & 0 \\
0 & 1
\end{array}\right)\left(\begin{array}{cc}
r^{-q} & 0 \\
0 & r^{-q}
\end{array}\right) \mathrm{N}_{E / F}\left(r^{2}\right), \phi^{\mathrm{N}_{E / F}\left(r^{-2}\right) a x z^{-1}}\right) \phi^{a}\left(y z^{-1}\right) \\
=\sum_{t \in F^{\times}} f\left(\phi^{t}\right) \pi_{1}\left(\left(\begin{array}{cc}
r^{-1} & 0 \\
0 & r^{-1}
\end{array}\right)\right) F_{t}\left(\left(\begin{array}{cc}
1 & 0 \\
0 & 1
\end{array}\right), \phi^{a x z^{-1}}\right) \phi^{a}\left(y z^{-1}\right) \\
=f\left(\phi^{a x z^{-1}}\right)\left[\pi_{1}\left(\left(\begin{array}{cc}
r^{-1} & 0 \\
0 & r^{-1}
\end{array}\right)\right) v_{1}\right] \phi^{a}\left(y z^{-1}\right) \\
=f\left(\phi^{a x z^{-1}}\right) \Lambda\left(r^{-1}\right) \Sigma\left(r^{-1}\right) v_{1} \phi^{a}\left(y z^{-1}\right)=f\left(\phi^{a x z^{-1}}\right) \Lambda\left(x^{-1}\right) \phi^{a}\left(y z^{-1}\right) v_{1} .
\end{gathered}
$$

So

$$
\pi_{0}\left(\left(\begin{array}{ll}
x & y \\
0 & z
\end{array}\right)\right) j(f)=\sum_{a \in F^{\times}} f\left(\phi^{a x^{-1} z}\right) \Lambda\left(x^{-1}\right) \phi^{a}\left(y z^{-1}\right) F_{a} .
$$

On the other hand,

$$
\begin{gathered}
j\left(\pi_{\Lambda^{-1}}\left(\left(\begin{array}{ll}
x & y \\
0 & z
\end{array}\right)\right) f\right)=\sum_{a \in F^{\times}}\left[\pi_{\Lambda^{-1}}\left(\left(\begin{array}{ll}
x & y \\
0 & z
\end{array}\right)\right) f\right]\left(\phi^{a}\right) F_{a} \\
=\sum_{a \in F^{\times}}\left[\pi_{\Lambda^{-1}}\left(\left(\begin{array}{cc}
x & 0 \\
0 & x
\end{array}\right)\left(\begin{array}{cc}
1 & 0 \\
0 & x^{-1} z
\end{array}\right)\left(\begin{array}{cc}
1 & x^{-1} y \\
0 & 1
\end{array}\right)\right) f\right]\left(\phi^{a}\right) F_{a} \\
=\sum_{a \in F^{\times}} \Lambda\left(x^{-1}\right) f\left(\phi^{a x z^{-1}}\right) \phi^{a x z^{-1}}\left(x^{-1} y\right) F_{a}
\end{gathered}
$$


(2) Let $g=\omega$.

$$
=\sum_{a \in F^{\times}} \Lambda\left(x^{-1}\right) f\left(\phi^{a x z^{-1}}\right) \phi^{a}\left(y z^{-1}\right) F_{a} .
$$

$$
\begin{aligned}
& \left(\pi_{0}(\omega) j(f)\right)\left(\xi_{a}\right)=-q^{-2} \sum_{n \in M} \phi^{a}\left(B\left(\operatorname{Id}_{H}, n\right)\right) j(f)\left(n, \phi^{a}\right) \\
& \stackrel{\text { consider supp }}{=}(j(f))-q^{-2} \sum_{n \in M, \operatorname{det} n \neq 0} \phi^{a}\left(B\left(\operatorname{Id}_{H}, n\right)\right) j(f)\left(n, \phi^{a}\right) \\
& =-q^{-2}\left|U_{2}(E)\right|^{-1} \sum_{h \in H} \phi^{a}\left(B\left(\operatorname{Id}_{H}, \mathrm{~N}_{E / F}\left(\operatorname{det}\left(h^{-1}\right)\right) h h^{\star}\right)\right) j(f)\left(\mathrm{N}_{E / F}\left(\operatorname{det}\left(h^{-1}\right)\right) h h^{\star}, \phi^{a}\right) \\
& =-q^{-2}\left|U_{2}(E)\right|^{-1} \sum_{h \in H} \phi^{a}\left(B\left(\operatorname{Id}_{H}, \mathrm{~N}_{E / F}\left(\operatorname{det}\left(h^{-1}\right)\right) h h^{\star}\right)\right) \pi_{1}(h) v_{1}\left[j(f)\left(\xi_{a \mathrm{~N}_{E / F}\left(\operatorname{det}\left(h^{-1}\right)\right)}\right)\right] \\
& =-q^{-2}\left|U_{2}(E)\right|^{-1} \sum_{h \in H} \phi^{a}\left(B\left(\operatorname{Id}_{H}, \mathrm{~N}_{E / F}\left(\operatorname{det}\left(h^{-1}\right)\right) h h^{\star}\right)\right) \pi_{1}(h) f\left(\phi^{a \mathrm{~N}_{E / F}\left(\operatorname{det}\left(h^{-1}\right)\right)}\right)
\end{aligned}
$$

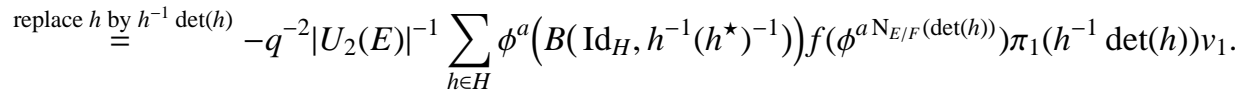

Let

$$
\kappa_{a}=-q^{-2}\left|U_{2}(E)\right|^{-1} \sum_{h \in H} \phi^{a}\left(B\left(\operatorname{Id}_{H}, h^{-1}\left(h^{\star}\right)^{-1}\right)\right) f\left(\phi^{a \mathrm{~N}_{E / F}(\operatorname{det}(h))}\right)\left(\pi_{1}\left(h^{-1} \operatorname{det}(h)\right) v_{1}\right)\left(1, e_{-1} ; 1\right)
$$

and

$\kappa_{a}^{s}=-q^{-2}\left|U_{2}(E)\right|^{-1} \sum_{h \in H, \mathrm{~N}_{E \mid F}(\operatorname{det}(h))=s} \phi^{a}\left(B\left(\operatorname{Id}_{H}, h^{-1}\left(h^{\star}\right)^{-1}\right)\right) f\left(\phi^{a \mathrm{~N}_{E / F}(\operatorname{det}(h))}\right)\left(\pi_{1}\left(h^{-1} \operatorname{det}(h)\right) v_{1}\right)\left(1, e_{-1} ; 1\right)$ for any $s \in F^{\times}$.

Then $\kappa_{a}=\sum_{s \in F^{\times}} \kappa_{a}^{s}$, and

$$
\begin{gathered}
\kappa_{a}^{1}=-q^{-2}\left|U_{2}(E)\right|^{-1} \sum_{h \in \mathcal{M}} \phi^{a}\left(\mathrm{~N}_{E / F}(\alpha)+\mathrm{N}_{E / F}(\beta)+\mathrm{N}_{E / F}(\gamma)+\mathrm{N}_{E / F}(\delta)\right) f\left(\phi^{a}\right) v_{1}\left(\delta-\gamma e_{-1},-\beta+\alpha e_{-1} ; \operatorname{det}\left(h^{-1}\right)\right) \\
=-q^{-2}\left|U_{2}(E)\right|^{-1} \sum_{h \in \mathcal{M}} \phi^{a}\left(\mathrm{~N}_{E / F}(\alpha)+\mathrm{N}_{E / F}(\beta)+\mathrm{N}_{E / F}(\gamma)+\mathrm{N}_{E / F}(\delta)\right) f\left(\phi^{a}\right) \Lambda^{q-1}\left(\delta-\gamma e_{-1}\right) \Lambda^{-q}(\alpha \delta-\beta \gamma) v_{1}\left(1, \frac{-\beta+\alpha e_{-1}}{\delta-\gamma e_{-1}} ; 1\right),
\end{gathered}
$$

where $\mathcal{M}=\left\{h=\left(\begin{array}{ll}\alpha & \beta \\ \gamma & \delta\end{array}\right) \in H \mid \mathrm{N}_{E / F}(\alpha \delta-\beta \gamma)=1 ;-\beta+\alpha e_{-1}=u_{1} e_{-1}\left(\delta-\gamma e_{-1}\right), \delta-\gamma e_{-1} \neq 0\right.$ for some $\left.u_{1} \in U\right\}$;

By the equations in $\left\{\alpha \delta-\beta \gamma=u_{2} ;-\beta+\alpha e_{-1}=u_{1} e_{-1}\left(\delta-\gamma e_{-1}\right)\right.$ and $\delta-\gamma e_{-1}=z$ for $\left.u_{1}, u_{2} \in U, z \in E^{\times}\right\}$, we change the variables $\alpha, \beta, \gamma, \delta$ by $u_{1}, u_{2}, z, \gamma$. Note that this is reasonable.

By $-\beta+\alpha e_{-1}=u_{1} e_{-1}\left(\delta-\gamma e_{-1}\right)$, we get $-\beta e_{-1}^{-1}+u_{1} \gamma e_{-1}=u_{1} \delta-\alpha$. Then $\left(-\beta e_{-1}^{-1}+u_{1} \gamma e_{-1}\right)\left(-\beta e_{-1}^{-1}+u_{1} \gamma e_{-1}\right)^{q}=$ $\left(u_{1} \delta-\alpha\right)\left(u_{1} \delta-\alpha\right)^{q}$. By calculation, we have $\mathrm{N}_{E / F}(\alpha)+\mathrm{N}_{E / F}(\beta)+\mathrm{N}_{E / F}(\gamma)+\mathrm{N}_{E / F}(\delta)=\operatorname{Tr}_{E / F}\left(u_{1}\left(\alpha^{q} \delta-\gamma \beta^{q} e_{-1}^{1-q}\right)\right)$. Set $A=\alpha^{q} \delta-\gamma \beta^{q} e_{-1}^{1-q}$. Now we consider

$$
u_{1} u_{2}^{-1} z=u_{1} u_{2}^{q} z=u_{1}\left(\alpha^{q} \delta^{q+1}-\beta^{q} \gamma^{q} \delta-\alpha^{q} \delta^{q} \gamma e_{-1}+\beta^{q} \gamma^{q+1} e_{-1}\right),
$$

and also

$$
\begin{gathered}
u_{1} A z^{q}=u_{1}\left[\left(\alpha^{q} \delta-\gamma \beta^{q} e_{-1}^{1-q}\right)\left(\delta^{q}-\gamma^{q} e_{-1}^{q}\right)\right] \\
=u_{1}\left[\alpha^{q} \delta^{q+1}-\alpha^{q} \delta \gamma^{q} e_{-1}^{q}-\delta^{q} \beta^{q} \gamma e_{-1}^{1-q}+\beta^{q} \gamma^{q+1} e_{-1}\right]=u_{1}\left[u_{2}^{-1} z+\beta^{q} \gamma^{q} \delta+\alpha^{q} \delta^{q} \gamma e_{-1}-\alpha^{q} \delta \gamma^{q} e_{-1}^{q}-\delta^{q} \beta^{q} \gamma e_{-1}^{1-q}\right] \\
=u_{1} u_{2}^{-1} z+u_{1} e_{-1}^{-q}\left(-\beta+\alpha e_{-1}\right)^{q}\left(\delta^{q} \gamma e_{-1}-\delta \gamma^{q} e_{-1}^{q}\right)=u_{1} u_{2}^{-1} z+z^{q}\left(\delta^{q} \gamma e_{-1}-\delta \gamma^{q} e_{-1}^{q}\right) .
\end{gathered}
$$

So

$$
u_{1} A=u_{1} u_{2}^{-1} z^{1-q}+\left(\delta^{q} \gamma e_{-1}-\delta \gamma^{q} e_{-1}^{q}\right) .
$$

In this way, we obtain

$$
\operatorname{Tr}_{E / F}\left(u_{1} A\right)=\operatorname{Tr}_{E / F}\left(u_{1} u_{2}^{-1} z^{1-q}\right) .
$$

Hence

$$
\underline{(3.8)}=-q^{-2}\left|U_{2}(E)\right|^{-1} \sum_{u_{1}, u_{2} \in U, z \in E^{\times}, \gamma \in E} \phi^{a}\left(\operatorname{Tr}_{E / F}\left(u_{1} u_{2}^{-1} z^{1-q}\right)\right) \Lambda^{-1}\left(u_{1} u_{2}^{-1} z^{1-q}\right) f\left(\phi^{a}\right)
$$




$$
\begin{gathered}
\stackrel{u_{1}=x_{1}^{1-q}, u_{2}=x_{2}^{q-1}}{=}-q^{-2}\left|U_{2}(E)\right|^{-1} \frac{1}{(q-1)^{2}} \sum_{x_{1}, x_{2}, z \in E^{\times}, \gamma \in E} \phi^{a}\left(\operatorname{Tr}_{E / F}\left(\left(x_{1} x_{2} z\right)^{1-q}\right)\right) \Lambda^{-1}\left(\left(x_{1} x_{2} z\right)^{1-q}\right) f\left(\phi^{a}\right) \\
=-\frac{1}{(q-1) q} \sum_{z \in E^{\times}} \phi^{a}\left(\operatorname{Tr}_{E / F}\left(z^{1-q}\right)\right) \Lambda^{-1}\left(z^{1-q}\right) f\left(\phi^{a}\right) \\
=-q^{-1} \sum_{y \in E^{\times}, \mathrm{N}_{E / F}(y)=1} \phi^{a}\left(\operatorname{Tr}_{E / F}(y)\right) \Lambda^{-1}(y) f\left(\phi^{a}\right) .
\end{gathered}
$$

Similarly we obtain

$$
\kappa_{a}^{s}=-q^{-1} \sum_{y \in E^{\times}, \mathrm{N}_{E / F}(y)=s} \phi^{a}\left(\operatorname{Tr}_{E / F}(y)\right) \Lambda^{-1}(y) f\left(\phi^{a s}\right) .
$$

Finally

$$
\kappa_{a}=-q^{-1} \sum_{y \in E^{\times}} \phi^{a}\left(\operatorname{Tr}_{E / F}(y)\right) \Lambda^{-1}(y) f\left(\phi^{a N_{E / F}(y)}\right) .
$$

Since $V_{1}^{U_{2}(E)}$ is one-dimensional, we have $\left(\pi_{0}(\omega) j(f)\right)\left(\xi_{a}\right)=j\left(\pi_{\Lambda^{-1}}(\omega) f\right)\left(\xi_{a}\right)$, which means $\pi_{0}(\omega) j(f)=$ $j\left(\pi_{\Lambda^{-1}}(\omega) f\right)$.

By the above (1), (2), we prove $\pi_{0} \simeq \pi_{\Lambda^{-1}}$.

3.7. By the above discussion I-IV about the representation $\left(\pi_{0}, W\left[\pi_{1}\right]\right)$, finally we achieve the main theorem in this section:

Theorem 3.16. For the representation $(\pi, G \times H, W)$, we have the following decomposition:

$$
\pi \simeq \bigoplus_{\sigma \in \operatorname{Irr}(G)} \sigma \otimes \mathrm{Bc}_{E / F}(\sigma) \oplus \bigoplus_{\psi \in \operatorname{Irr}\left(F^{\times}\right), \Psi \in \operatorname{Irr}\left(E^{\times}\right), \Psi=\psi \circ \mathrm{N}_{E / F}} \psi \mathrm{St}_{G} \otimes \Psi \cdot 1_{H} .
$$

4. The Decomposition of the WeIL REPRESENTATION OF $\mathrm{GL}_{2}(K)$

4.1. In this section, we use the following notations: $G=\mathrm{GL}_{2}(K), B=\left\{\left(\begin{array}{ll}a & b \\ 0 & d\end{array}\right) \in G\right\}, N=\left\{\left(\begin{array}{ll}1 & b \\ 0 & 1\end{array}\right) \in G\right\}, T=$ $\left\{\left(\begin{array}{ll}a & 0 \\ 0 & d\end{array}\right) \in G\right\}, Z=\left\{\left(\begin{array}{cc}a & 0 \\ 0 & a\end{array}\right) \in G\right\} ; \operatorname{Gal}(K / F)=\langle\sigma\rangle ; u(b)=\left(\begin{array}{cc}1 & b \\ 0 & 1\end{array}\right)$ for $b \in K, h(a, d)=\left(\begin{array}{ll}a & 0 \\ 0 & d\end{array}\right)$ for $a, d \in K^{\times}$, $\omega=\left(\begin{array}{cc}0 & 1 \\ -1 & 0\end{array}\right) \in G$

4.2. We recall the technique of Weil's Galois descent to construct a morphism from $G$ to $\operatorname{GSp}_{8}(F)$.

Let $V_{0}$ be a vector space over $F$ of dimension 2 , endowed with a symplectic form $\langle,\rangle_{V_{0}}$. Let $\left\{e_{1}, e_{2}\right\}$ be a symplectic base of $V_{0}$. Namely $V=V_{0} \otimes_{F} K$ is a symplectic $K$-vector space, endowed with the symplectic form $\langle,\rangle_{V}$ induced from $V_{0}$ by scalar extension. Let us define a $\mathrm{Gal}(K / F)$-action on $V$ by

$$
\operatorname{Gal}(K / F) \times K \otimes_{F} V_{0} \longrightarrow K \otimes_{F} V_{0} ;\left(\sigma, \sum_{i} k_{i} \otimes e_{i}\right) \longmapsto \sum_{i} k_{i}^{\sigma} \otimes e_{i}
$$

Let $W=V \otimes_{K} V \otimes_{K} V$, and we assign $W$ a symplectic form $\langle,\rangle_{W}=\langle,\rangle_{V} \otimes\langle,\rangle_{V} \otimes\langle,\rangle_{V}$. On $W$, we will consider the twisted Galois action defined by

$$
\operatorname{Gal}(K / F) \times W \longrightarrow W ;\left(\sigma, w=\sum_{i=1}^{n} u_{i} \otimes v_{i} \otimes w_{i}\right) \longmapsto{ }^{\sigma} w=\sum_{i=1}^{n} w_{i}^{\sigma} \otimes u_{i}^{\sigma} \otimes v_{i}^{\sigma}
$$

We will let $W_{0}$ denote the set $\left\{\left.w \in W\right|^{\sigma} w=w\right\}$. By calculation, each $w_{0} \in W_{0}$ may be expressed in the form

$$
\begin{gathered}
w_{0}=x e_{1} \otimes e_{1} \otimes e_{1}+\alpha e_{1} \otimes e_{1} \otimes e_{2}+\alpha^{\sigma} e_{2} \otimes e_{1} \otimes e_{1}+\alpha^{\sigma^{2}} e_{1} \otimes e_{2} \otimes e_{1} \\
+\beta^{\sigma^{2}} e_{2} \otimes e_{1} \otimes e_{2}+\beta^{\sigma} e_{1} \otimes e_{2} \otimes e_{2}+\beta e_{2} \otimes e_{2} \otimes e_{1}+y e_{2} \otimes e_{2} \otimes e_{2} \text { for } x, y \in F, \alpha, \beta \in K .
\end{gathered}
$$


Every element $w_{0}$ of this form is well-defined by its corresponding coefficients. For simplicity, we write $w_{0}=$ $\left(\begin{array}{ll}x & \alpha \\ \beta & y\end{array}\right)$ instead of the whole term. One can check that the restriction of $\langle,\rangle_{W}$ to $W_{0}$ defines an $F$-symplectic form, denoted by $\langle,\rangle_{W_{0}}$. More precisely,

$$
\left\langle w_{0}, w_{0}^{\prime}\right\rangle_{W_{0}}=x y^{\prime}-x^{\prime} y-\operatorname{Tr}_{K / F}\left(\alpha \beta^{\prime}\right)+\operatorname{Tr}_{K / F}\left(\alpha^{\prime} \beta\right) \text { for } w_{0}=\left(\begin{array}{ll}
x & \alpha \\
\beta & y
\end{array}\right), w_{0}^{\prime}=\left(\begin{array}{ll}
x^{\prime} & \alpha^{\prime} \\
\beta^{\prime} & y^{\prime}
\end{array}\right) .
$$

Let $\mathrm{GSp}(W)$ denote the group of symplectic similitudes of $\left(W,\langle,\rangle_{W}\right)$. By definition, there actually exists a morphism of groups

$$
(\mathrm{GL}(V) \times \mathrm{GL}(V) \times \mathrm{GL}(V)) \rtimes S_{3} \longrightarrow \mathrm{GSp}(W) .
$$

Here the group $S_{3}$ acts on $W$ by permutating its three variables. Now we define a twisted Galois action of $\operatorname{Gal}(K / F)$ on $\mathrm{GL}(V) \times \mathrm{GL}(V) \times \mathrm{GL}(V)$ by

$$
\operatorname{Gal}(K / F) \times(\mathrm{GL}(V) \times \mathrm{GL}(V) \times \mathrm{GL}(V)) \longrightarrow \mathrm{GL}(V) \times \mathrm{GL}(V) \times \mathrm{GL}(V) ; h=\left(g_{1}, g_{2}, g_{3}\right) \longmapsto{ }^{\sigma} h:=\left(g_{3}^{\sigma}, g_{1}^{\sigma}, g_{2}^{\sigma}\right) .
$$

Write $\overline{\mathrm{GL}(V)}=\left\{h \in \mathrm{GL}(V) \times \mathrm{GL}(V) \times\left.\mathrm{GL}(V)\right|^{\sigma} h=h\right\}$. Then there exists an isomorphism of groups $\operatorname{GL}(V) \longrightarrow$ $\overline{\mathrm{GL}(V)} ; g \longmapsto\left(g, g^{\sigma}, g^{\sigma^{2}}\right)$. If given $h \in \mathrm{GL}(V) \times \mathrm{GL}(V) \times \mathrm{GL}(V), \quad w \in W=V \otimes_{K} V \otimes_{K} V$, one can verify that ${ }^{\sigma} h \cdot{ }^{\sigma} w={ }^{\sigma}(h \cdot w)$. So it induces a morphism from $\operatorname{GL}(V) \simeq \overline{\operatorname{GL}(V)}$ to $\operatorname{GSp}\left(W_{0}\right)$. By the fixed basis $\left\{e_{1}, e_{2}\right\}$, we obtain a morphism: $G \stackrel{i}{\longrightarrow} \operatorname{GSp}\left(W_{0}\right)$.

4.3. We interpret the above construction of the morphism $G \stackrel{i}{\longrightarrow} \mathrm{GSp}\left(W_{0}\right)$ in terms of the language of algebraic groups.

Let $\mathbf{V}$ be the $K$-algebraic vector space associated to $V$. That is to say:

$$
\mathbf{V}: \mathbf{A l g}_{K} \longrightarrow \operatorname{Vect}_{K} ; R \longmapsto V \otimes_{K} R,
$$

a functor from the category of unital commutative associative $K$-algebras to the category of $K$-vector spaces. Namely $V \otimes_{K} R$ inherits the $R$-symplectic structure from $V$. We define a $\operatorname{Gal}(K / F)$-action on $\mathbf{V}$ in the following way:

$$
\operatorname{Gal}(K / F) \times V \otimes_{K} R \longrightarrow V \otimes_{K} R ;\left(\sigma, \sum_{i=1}^{n} v_{i} \otimes r_{i}\right) \longmapsto \sum_{i=1}^{n} v_{i}^{\sigma} \otimes r_{i}^{\sigma}
$$

Now let $\mathbf{W}$ be the $K$-algebraic vector space associated to $W$, and $\mathbf{W}_{0}$ the $F$-algebraic vector space associated to $W_{0}$. We define a twisted $\operatorname{Gal}(K / F)$-action on $\mathbf{W}$ in the following way:

$$
\begin{gathered}
\operatorname{Gal}(K / F) \times V \otimes_{K} V \otimes_{K} V \otimes_{K} R \longrightarrow V \otimes_{K} V \otimes_{K} V \otimes_{K} R \\
\left(\sigma, \sum_{i=1}^{n} u_{i} \otimes v_{i} \otimes w_{i} \otimes r_{i}\right) \\
\longmapsto \sum_{i=1}^{n} w_{i}^{\sigma} \otimes u_{i}^{\sigma} \otimes v_{i}^{\sigma} \otimes r_{i}^{\sigma} .
\end{gathered}
$$

So $\mathbf{W}_{0}$ is the $\operatorname{Gal}(K / F)$-invariant algebraic scheme of $\mathbf{W}$ in the following sense:

(1) $\mathbf{W} \simeq \mathbf{W}_{0} \times_{F} K$.

(2) $\mathbf{W}(R)^{\mathrm{Gal}(K / F)} \simeq \mathbf{W}_{0}\left(R^{\mathrm{Gal}(K / F)}\right)$ for any $R \in \mathbf{A} \mathbf{I g}_{K}$.

On the other hand, we also define a twisted Galois action of $\operatorname{Gal}(K / F)$ on $\mathbf{G L}_{2 / K} \times \mathbf{G L}_{2 / K} \times \mathbf{G L}_{2 / K}$ as

$$
\begin{gathered}
\mathrm{Gal}(K / F) \times\left(\mathrm{GL}_{2}(R) \times \mathrm{GL}_{2}(R) \times \mathrm{GL}_{2}(R)\right) \longrightarrow \mathrm{GL}_{2}(R) \times \mathrm{GL}_{2}(R) \times \mathrm{GL}_{2}(R) ; \\
\left(\sigma,\left(g_{1}, g_{2}, g_{3}\right)\right) \longmapsto\left(g_{3}^{\sigma}, g_{2}^{\sigma}, g_{1}^{\sigma}\right) .
\end{gathered}
$$

We denote by $\mathbf{H}^{\mathrm{Gal}(K / F)}$, the $\operatorname{Gal}(K / F)$-invariant algebraic scheme of $\mathbf{H}=\mathbf{G L}_{2 / K} \times \mathbf{G L}_{2 / K} \times \mathbf{G} \mathbf{L}_{2 / K}$. Indeed, by definition,

$$
\mathbf{H}^{\mathrm{Gal}(K / F)} \simeq \operatorname{Res}_{K / F}\left(\mathbf{G L}_{2 / K}\right) .
$$

There exists an action of $\mathbf{H}^{\mathrm{Gal}(K / F)}$ on $\mathbf{W}_{0}$, and it preserves the symplectic form up to the similitude factors. Thus we obtain a morphism of algebraic group schemes:

$$
\mathbf{i}: \operatorname{Res}_{K / F}\left(\mathbf{G L}_{2 / K}\right) \longrightarrow \mathbf{G S p}_{W_{0}} .
$$


4.4. Let $X_{0}=\left\{w_{0}=\left(\begin{array}{cc}x & \alpha \\ 0 & 0\end{array}\right) \mid w_{0} \in W_{0}\right\}, Y_{0}=\left\{w_{0}=\left(\begin{array}{cc}0 & 0 \\ \beta & y\end{array}\right) \mid w_{0} \in W_{0}\right\}$. Then $X_{0}, Y_{0}$ are two vector spaces over $F$ and $W_{0}=X_{0} \oplus Y_{0}$ is a complete polarization of $W_{0}$. Via the morphism $i: G \longrightarrow \mathrm{GSp}\left(W_{0}\right)$, it gives rise to a $G$-action on $W_{0}$ by the following formulas:

$$
\begin{aligned}
& \text { For } g=\left(\begin{array}{ll}
a & b \\
c & d
\end{array}\right) \in G, w_{0}=\left(\begin{array}{ll}
x & \alpha \\
\beta & y
\end{array}\right) \text {, write } g \cdot w_{0}=\left(\begin{array}{ll}
x^{\prime} & \alpha^{\prime} \\
\beta^{\prime} & y^{\prime}
\end{array}\right) \text {. Then } \\
& x^{\prime}=\mathrm{N}_{K / F}(a) x+\operatorname{Tr}_{K / F}\left(a a^{\sigma} b^{\sigma^{2}} \alpha\right)+\operatorname{Tr}_{K / F}\left(b b^{\sigma} a^{\sigma^{2}} \beta\right)+\mathrm{N}_{K / F}(b) y ; \\
& \alpha^{\prime}=a a^{\sigma} c^{\sigma^{2}} x+\left(a a^{\sigma} d^{\sigma^{2}} \alpha+b a^{\sigma} c^{\sigma^{2}} \alpha^{\sigma}+a b^{\sigma} c^{\sigma^{2}} \alpha^{\sigma^{2}}\right)+\left(b b^{\sigma} c^{\sigma^{2}} \beta+a b^{\sigma} d^{\sigma^{2}} \beta^{\sigma}+b a^{\sigma} d^{\sigma^{2}} \beta^{\sigma^{2}}\right)+b b^{\sigma} d^{\sigma^{2}} y ; \\
& \beta^{\prime}=d d^{\sigma} b^{\sigma^{2}} y+\left(d d^{\sigma} a^{\sigma^{2}} \beta+c d^{\sigma} b^{\sigma^{2}} \beta^{\sigma}+d c^{\sigma} b^{\sigma^{2}} \beta^{\sigma^{2}}\right)+\left(c c^{\sigma} b^{\sigma^{2}} \alpha+d c^{\sigma} a^{\sigma^{2}} \alpha^{\sigma}+c d^{\sigma} a^{\sigma^{2}} \alpha^{\sigma^{2}}\right)+c c^{\sigma} a^{\sigma^{2}} x ; \\
& y^{\prime}=\mathrm{N}_{K / F}(d) y+\operatorname{Tr}_{K / F}\left(d d^{\sigma} c^{\sigma^{2}} \beta\right)+\operatorname{Tr}_{K / F}\left(c c^{\sigma} d^{\sigma^{2}} \alpha\right)+\mathrm{N}_{K / F}(c) x .
\end{aligned}
$$

We write each element $h \in \operatorname{GSp}\left(W_{0}\right)$ in the form of $h=\left(\begin{array}{ll}a & b \\ c & d\end{array}\right)$ with $a \in \operatorname{End}_{F}\left(X_{0}\right), b \in \operatorname{Hom}_{F}\left(Y_{0}, X_{0}\right), c \in$ $\operatorname{Hom}_{F}\left(X_{0}, Y_{0}\right), d \in \operatorname{End}_{F}\left(Y_{0}\right)$.

Corollary 4.1. Through the map $i: G \longrightarrow \operatorname{GSp}\left(W_{0}\right)$, the actions of $u(b), h(a, d), \omega$ on $W_{0}$ are described as follows:

(1) $i(u(b))=\left(\begin{array}{cc}m & n \\ 0 & m^{\vee}\end{array}\right)$, where $m\left(\begin{array}{cc}x & \alpha \\ 0 & 0\end{array}\right)=\left(\begin{array}{cc}x+\operatorname{Tr}_{K / F}\left(b^{\sigma^{2}} \alpha\right) & \alpha \\ 0 & 0\end{array}\right)$,

$$
n\left(\begin{array}{ll}
0 & 0 \\
\beta & y
\end{array}\right)=\left(\begin{array}{cc}
\operatorname{Tr}_{K / F}\left(b b^{\sigma} \beta\right)+\mathrm{N}_{K / F}(b) y & b^{\sigma} \beta^{\sigma}+b \beta^{\sigma^{2}}+b b^{\sigma} y \\
0 & 0
\end{array}\right), m^{\vee}\left(\begin{array}{ll}
0 & 0 \\
\beta & y
\end{array}\right)=\left(\begin{array}{cc}
0 & 0 \\
\beta+b^{\sigma^{2}} y & y
\end{array}\right) ;
$$

(2) $i(h(a, d))=\left(\begin{array}{cc}m & 0 \\ 0 & n\end{array}\right)$, where $m\left(\begin{array}{cc}x & \alpha \\ 0 & 0\end{array}\right)=\left(\begin{array}{cc}\mathrm{N}_{K / F}(a) x & a a^{\sigma} d^{\sigma^{2}} \alpha \\ 0 & 0\end{array}\right), n\left(\begin{array}{ll}0 & 0 \\ \beta & y\end{array}\right)=\left(\begin{array}{cc}0 & 0 \\ d d^{\sigma} a^{\sigma^{2}} \beta & \mathrm{N}_{K / F}(d) y\end{array}\right)$;

(3) $i(\omega)=\left(\begin{array}{ll}0 & u \\ v & 0\end{array}\right)$, where $u\left(\begin{array}{ll}0 & 0 \\ \beta & y\end{array}\right)=\left(\begin{array}{cc}y & -\beta \\ 0 & 0\end{array}\right), v\left(\begin{array}{cc}x & \alpha \\ 0 & 0\end{array}\right)=\left(\begin{array}{cc}0 & 0 \\ \alpha & -x\end{array}\right)$.

Let $(\rho, V)$ be the Weil representation of the symplectic similitude group $\operatorname{GSp}\left(W_{0}\right)$. Via the map $i$, it gives rise to a representation $(\pi, V)$ of $G$ which can be realized in the vector space $V=\mathbb{C}\left[Y_{0} \times X_{F}\right]$ of complex functions on $Y_{0} \times X_{F}$

Proposition 4.2. For the representation $\left(\pi, G, \mathbb{C}\left[Y_{0} \times X_{F}\right]\right)$, the action is determined by the following formulas:

(1) $[\pi(u(b)) F]\left(\left(\begin{array}{ll}0 & 0 \\ \beta & y\end{array}\right), \psi\right)=\psi\left(\operatorname{Tr}_{K / F}\left(b b^{\sigma} \beta y\right)-\mathrm{N}_{K / F}(b) y^{2}-\operatorname{Tr}_{K / F}\left(b \beta \beta^{\sigma^{2}}\right)\right) F\left(\left(\begin{array}{cc}0 & 0 \\ \beta-b^{\sigma^{2}} y & y\end{array}\right), \psi\right)$;

(2) $[\pi(h(a, d)) F]\left(\left(\begin{array}{ll}0 & 0 \\ \beta & y\end{array}\right), \psi\right)=\chi_{q}^{+}\left(\mathrm{N}_{K / F}(a d)\right) F\left(\left(\begin{array}{cc}0 & 0 \\ \frac{\mathrm{N}_{K / F}(a d)}{d d^{\sigma} a^{\sigma^{2}}} \beta & \mathrm{N}_{K / F}(a) y\end{array}\right), \psi^{\mathrm{N}_{K / F}(a d)^{-1}}\right)$;

(3) $[\pi(\omega) F]\left(\left(\begin{array}{ll}0 & 0 \\ \beta & y\end{array}\right), \psi\right)=q^{-2} \sum_{\beta^{\prime} \in K, y^{\prime} \in F} F\left(\left(\begin{array}{cc}0 & 0 \\ \beta^{\prime} & y^{\prime}\end{array}\right), \psi\right) \psi\left(y y^{\prime}+\operatorname{Tr}_{K / F}\left(\beta \beta^{\prime}\right)\right)$.

Proof. See Appendix 1.

4.5. The whole goal of this section is to determine the different isotypic components of $\pi$. We first consider the principal series representations.

Let $\alpha, \beta \in \operatorname{Irr}\left(K^{\times}\right)$. To determine the principal series components of $\pi$, it involves to calculate the dimension of the vector space $\operatorname{Hom}_{G}\left(V, \operatorname{Ind}_{B}^{G}(\alpha \otimes \beta)\right)$. Applying Frobenius reciprocity, we see

$$
\operatorname{Hom}_{G}\left(V, \operatorname{Ind}_{B}^{G}(\alpha \otimes \beta)\right) \simeq \operatorname{Hom}_{T}\left(V_{N}, \alpha \otimes \beta\right) \simeq \operatorname{Hom}_{T}\left(V^{N}, \alpha \otimes \beta\right) .
$$

Therefore we shall first describe the vector space $V^{N}$, and then consider the $T$-action on it. Once we regard the action of $N$ on the vector space $V$, as described in Proposition 4.2(1), we should consider the following action:

$$
N \times\left(Y_{0} \times X_{F}\right) \longrightarrow Y_{0} \times X_{F} ;\left(\left(\begin{array}{ll}
1 & b \\
0 & 1
\end{array}\right),\left(\left(\begin{array}{ll}
0 & 0 \\
\beta & y
\end{array}\right), \psi\right)\right) \longmapsto\left(\left(\begin{array}{cc}
0 & 0 \\
\beta-b^{\sigma^{2}} y & y
\end{array}\right), \psi\right)
$$

The orbits of this action are following:

(i) $\operatorname{Orbit}\left\{\xi_{(\beta, 0 ; \psi)}\right\}$, where $\xi_{(\beta, 0 ; \psi)}=\left(\left(\begin{array}{cc}0 & 0 \\ \beta & 0\end{array}\right), \psi\right)$ for any $\beta \in K, \psi \in X_{F}$;

(ii) Orbit $\left\{\eta_{(0, y ; \psi)}\right\}$, where $\eta_{(0, y ; \psi)}=\left(\left(\begin{array}{ll}0 & 0 \\ 0 & y\end{array}\right), \psi\right)$ for any $y \in F^{\times}, \psi \in X_{F}$. 
The stabilizer of the chosen element in each orbit is described as follows:

$$
\operatorname{Stab}_{N}\left(\xi_{(\beta, 0 ; \psi)}\right)=N \quad \text { and } \quad \operatorname{Stab}_{N}\left(\eta_{(0, y ; \psi)}\right)=1_{N} .
$$

A function $F$ belongs to $V^{N}$ if and only if it satisfies the equality:

$$
\psi\left(\operatorname{Tr}_{K / F}\left(b b^{\sigma} \beta y\right)-\mathrm{N}_{K / F}(b) y^{2}-\operatorname{Tr}_{K / F}\left(b \beta \beta^{\sigma^{2}}\right)\right) F\left(\left(\begin{array}{cc}
0 & 0 \\
\beta-b^{\sigma^{2}} y & y
\end{array}\right), \psi\right)=F\left(\left(\begin{array}{ll}
0 & 0 \\
\beta & y
\end{array}\right), \psi\right)
$$

for any $b \in K$.

Proposition 4.3. (1) The vector space $V^{N}$ is generated by the following functions:

(i) $F_{(0,0 ; \psi)}$, where $\operatorname{supp}\left(F_{(0,0 ; \psi)}\right)=$ Orbit $\left\{\xi_{(0,0 ; \psi)}\right\}, F_{(0,0, \psi)}\left(\xi_{(0,0 ; \psi)}\right)=1$ and it satisfies the equation (4.1) for any $\psi \in X_{F}$;

(ii) $G_{(0, y ; \psi)}$, where $\operatorname{supp}\left(G_{(0, y ; \psi)}\right)=$ Orbit $\left\{\eta_{(0, y ; \psi)}\right\}, G_{(0, y ; \psi)}\left(\eta_{(0, y ; \psi)}\right)=1$ and it satisfies the equation (4.1) for any $y \in F^{\times}$, any $\psi \in X_{F}$.

(2) Let $t=h(a, d) \in T$. Then the action of $t$ on the vector space $V^{N}$ is given as follows:

(i) $\pi(t) F_{(0,0 ; \psi)}=\chi_{q}^{+}\left(\mathrm{N}_{K / F}(a d)\right) F_{\left(0,0 ; \psi^{\mathrm{N}_{K / F}(a d)}\right)}$;

(ii) $\left.\pi(t) G_{(0, y ; \psi)}=\chi_{q}^{+}\left(\mathrm{N}_{K / F}(a d)\right) G_{\left(0, \frac{1}{\mathrm{~N}_{K / F}(a)}\right.} y ; \psi^{\mathrm{N}_{K / F}(a d)}\right)$.

Proof. 1) Every element $F$ in $V^{N}$, that satisfies the equation (4.1), is completely determined by its values at the points in $\left\{\xi_{(\beta, 0 ; \psi)}, \eta_{(0, y ; \psi)}\right\}$. Let $\delta$ be one point among them. Then $F(\delta)$ can be nonzero if and only if the coefficient on the left-hand side of the equation (4.1) is trivial over the stabilizer of $\delta$, After checking each such point, we obtain the result.

2) It is straightforward.

Let $\Phi$ be an element in $\operatorname{Hom}_{T}\left(V^{N}, \alpha \otimes \beta\right)$. Then it is determined by the following two equations:

(1) $\chi_{q}^{+}\left(\mathrm{N}_{K / F}(a d)\right) \Phi\left(F_{\left(0,0 ; \psi^{\mathrm{N}_{K / F}(a d)}\right)}\right)=\alpha(a) \beta(b) \Phi\left(F_{(0,0 ; \psi)}\right), \quad a, d \in K^{\times}$.

(2) $\chi_{q}^{+}\left(\mathrm{N}_{K / F}(a d)\right) \Phi\left(G_{\left(0, \frac{1}{E_{E / F^{(a)}}} y ; \psi^{\mathrm{N} K / F^{(a d)}}\right)}\right)=\alpha(a) \beta(b) \Phi\left(G_{(0, y ; \psi)}\right), \quad a, d \in K^{\times}$.

Now let us define a $T$-action on the vector space $V^{N}$ :

$$
\left.t \cdot F_{(0,0 ; \psi)}:=F_{\left(0,0 ; \psi^{\mathrm{N} K / F}(a d)\right.}\right) \quad \text { and } \quad t \cdot G_{(0, y ; \psi)}=G_{\left(0, \frac{1}{N_{E / F}(a)} y ; \psi^{\mathrm{N}_{E / F}(a d)}\right)}, \quad t=\left(\begin{array}{ll}
a & 0 \\
0 & d
\end{array}\right) .
$$

For such action, there are two kinds of orbits:

$$
\text { (i) } \operatorname{Orbit}\left\{F_{(0,0 ; \phi)}\right\} \quad \text { and } \quad \text { (ii) } \operatorname{Orbit}\left\{G_{(0,1 ; \phi)}\right\}, \quad \text { for the fixed } \phi \in X_{F} \text {. }
$$

The stabilizer of the representative element in each orbit has the following form:

(i) $\operatorname{Stab}_{T}\left(F_{(0,0 ; \phi)}\right)=\left\{h(a, d) \in T \mid \mathrm{N}_{K / F}(a d)=1\right\}$;

(ii) $\operatorname{Stab}_{T}\left(G_{(0,1 ; \phi)}\right)=\left\{h(a, d) \in T \mid \mathrm{N}_{K / F}(a)=\mathrm{N}_{K / F}(d)=1\right\}$.

Now we present one statement about the principal series components of the representation $\pi$ :

Proposition 4.4. Let $\alpha, \beta \in \operatorname{Irr}\left(K^{\times}\right)$.

(1) If $\alpha=\chi_{1} \circ \mathrm{N}_{K / F}, \beta=\chi_{2} \circ \mathrm{N}_{K / F}$ for some characters $\chi_{1} \neq \chi_{2} \in \operatorname{Irr}\left(F^{\times}\right)$, then $\operatorname{dim}_{\mathbb{C}} \operatorname{Hom}_{G}\left(V, \operatorname{Ind}_{B}^{G}(\alpha \otimes \beta)\right)=1$.

(2) If $\alpha=\beta=\chi \circ \mathrm{N}_{K / F}$ for a character $\chi \in \operatorname{Irr}\left(F^{\times}\right)$, then $\operatorname{dim}_{\mathbb{C}} \operatorname{Hom}_{G}\left(V, \operatorname{Ind}_{B}^{G}\left(\alpha \cdot 1_{B}\right)\right)=2$.

For the other kind of $\alpha, \beta \in \operatorname{Irr}\left(K^{\times}\right), \operatorname{Hom}_{G}\left(V, \operatorname{Ind}_{B}^{G}(\alpha \otimes \beta)\right)=0$.

Proof. By Frobenius reciprocity, we see $\operatorname{Hom}_{G}\left(V, \operatorname{Ind}_{B}^{G}(\alpha \otimes \beta)\right) \simeq \operatorname{Hom}_{T}\left(V^{N}, \alpha \otimes \beta\right)$. Let $\Phi \in \operatorname{Hom}_{T}\left(V^{N}, \alpha \otimes \beta\right)$. The function $\Phi$ is completely determined by its values at the points $F_{(0,0 ; \phi)}$ and $G_{(0,1 ; \phi)}$. The value $\Phi\left(F_{(0,0 ; \phi)}\right)$ can be any complex number if and only if $\alpha \otimes \beta(t)=1$ for $t=h(a, d) \in \operatorname{Stab}_{T}\left(F_{(0,0 ; \phi)}\right)$, which is equivalent to $\alpha=\beta=\chi \circ \mathrm{N}_{K / F}$ for some character $\chi \in \operatorname{Irr}\left(F^{\times}\right)$. Similarly the value $\Phi\left(G_{(0,1 ; \phi)}\right)$ can be any complex number if and only if $\alpha=\chi_{1} \circ \mathrm{N}_{K / F}, \beta=\chi_{2} \circ \mathrm{N}_{K / F}$ for two characters $\chi_{1}, \chi_{2} \in \operatorname{Irr}\left(F^{\times}\right)$; thus we obtain the results. 
4.6. Now it reduces to check whether the representation $\chi \circ \mathrm{N}_{E / F} \cdot 1_{G}$ of $G$ is a sub-representation of $\pi$.

Let $\left(\alpha \cdot \pi, V_{\alpha}\right)$ be the representation of $\pi$ twisted by the character $\alpha=\chi \circ \mathrm{N}_{K / F} \in \operatorname{Irr}(G)$. Since $\operatorname{Hom}_{G}\left(\pi, \alpha^{-1} \cdot 1_{G}\right) \simeq$ $\left(V_{\alpha}\right)^{G}$, it suffices to determine the dimension of $\left(V_{\alpha}\right)^{G}$ for the representation $\left(\alpha \cdot \pi, G, V_{\alpha}\right)$. Notice that $\left(V_{\alpha}\right)^{N} \simeq V^{N}$ which is generated by two functions $F_{(0,0 ; \psi)}, G_{(0, y ; \psi)}$; the action of $T$ on $\left(V_{\alpha}\right)^{N}$ is given by the following formulas:

(1) $[\alpha \cdot \pi](h(a, d)) F_{(0,0 ; \psi)}=\chi \cdot \chi_{q}^{+}\left(\mathrm{N}_{K / F}(a d)\right) F_{\left(0,0 ; \psi^{\mathrm{N}} / F(a d)\right.}$;

(2) $\left.[\alpha \cdot \pi](h(a, d)) G_{(0, y ; \psi)}=\chi \cdot \chi_{q}^{+}\left(\mathrm{N}_{K / F}(a d)\right) G_{\left(0, \frac{1}{\mathrm{~N}_{K / F}(a)}\right.} y ; \psi^{\mathrm{N}_{K / F}(a d)}\right) \cdot$

Proposition 4.5. The vector space $\left(V_{\alpha}\right)^{B}$ is generated by two non-zero functions $A=\sum_{t \in T} \alpha \cdot \pi(t) F_{(0,0 ; \phi)}$ and $B=\sum_{t \in T} \alpha \cdot \pi(t) G_{(0,1 ; \phi)}$ for the fixed $\phi \in X_{F}$.

Proof. It is straightforward.

Our final task for this subsection is to consider the action of $\omega$ on the vector space $\left(V_{\alpha}\right)^{B}$. Observe that $[\alpha$. $\pi](\omega) A,[\alpha \cdot \pi](\omega) B$ both belong to $\left(V_{\alpha}\right)^{T}$. Consider the $T$-action on the set $Y_{0} \times X_{F}$ : ( We treat the vector space $\left(V_{\alpha}\right)^{T}$ similarly as $\left(V_{\alpha}\right)^{N}$.)

$$
T \times\left(Y_{0} \times X_{F}\right) \longrightarrow Y_{0} \times X_{F} ;\left(\left(\begin{array}{ll}
a & 0 \\
0 & d
\end{array}\right),\left(\left(\begin{array}{ll}
0 & 0 \\
\beta & y
\end{array}\right), \psi\right)\right) \longmapsto\left(\left(\begin{array}{cc}
0 & 0 \\
\frac{\mathrm{N}_{K / F}(a d)}{d d^{\sigma} a^{\sigma^{2}}} \beta & \mathrm{N}_{K / F}(a) y
\end{array}\right), \psi^{\mathrm{N}_{K / F}(a d)^{-1}}\right)
$$

The orbits of this action are following:

$$
\begin{aligned}
& \text { Orbit }\left\{x_{00}\right\}, x_{00}=\left(\left(\begin{array}{ll}
0 & 0 \\
0 & 0
\end{array}\right), \phi\right) \text { and Orbit }\left\{x_{10}\right\}, x_{10}=\left(\left(\begin{array}{ll}
0 & 0 \\
1 & 0
\end{array}\right), \phi\right), \\
& \text { Orbit }\left\{x_{01}\right\}, x_{01}=\left(\left(\begin{array}{ll}
0 & 0 \\
0 & 1
\end{array}\right), \phi\right) \text { and Orbit }\left\{y_{k}\right\}, y_{k}=\left(\left(\begin{array}{ll}
0 & 0 \\
1 & 1
\end{array}\right), \phi^{k}\right)
\end{aligned}
$$

for the fixed character $\phi \in X_{F}$ and any $k \in F^{\times}$. By the calculations in Appendix 2, we obtain the following table for the values of the functions $A, B,[\alpha \cdot \pi](\omega) A,[\alpha \cdot \pi](\omega) B$ at the points (1) $x_{00}$; (2) $x_{01}$; (3) $x_{10}$; (4) $y_{k}$.

\begin{tabular}{|l|l|l|l|l|}
\hline & $x_{00}$ & $x_{01}$ & $x_{10}$ & $y_{k}$ \\
\hline $\mathrm{A}$ & $(q-1)\left(q^{2}+q+1\right)^{2}$ & 0 & 0 & 0 \\
\hline $\mathrm{B}$ & 0 & $\left(q^{2}+q+1\right)^{2}$ & 0 & $\begin{array}{l}\chi \chi_{q}^{+}(k) \phi(-k)\left(q^{2}+q+\right. \\
1)^{2}\end{array}$ \\
\hline$\alpha \cdot \pi(\omega) A$ & $q^{-2}(q-1)\left(q^{2}+q+1\right)^{2}$ & $q^{-2}(q-1)\left(q^{2}+q+1\right)^{2}$ & $q^{-2}(q-1)\left(q^{2}+q+1\right)^{2}$ & $\begin{array}{l}\chi \chi_{q}^{+}(k) q^{-2}(q-1)\left(q^{2}+\right. \\
q+1)^{2}\end{array}$ \\
\hline$\alpha \cdot \pi(\omega) B$ & $\begin{array}{l}-q^{-1}(q-1)(q+1)\left(q^{2}+\right. \\
q+1)^{2}\end{array}$ & $q^{-1}(q+1)\left(q^{2}+q+1\right)^{2}$ & $q^{-1}\left(q^{2}+q+1\right)^{2}$ & \\
\hline
\end{tabular}

Corollary 4.6. The element $q A-(q-1) B \in V_{\alpha}^{B}$ is $[\alpha \cdot \pi](\omega)$-invariant.

Proof. Let us consider $C=\sum_{g \in G}[\alpha \cdot \pi](g) F_{(0,0 ; \phi)}$. Then

$$
\begin{gathered}
C\left(x_{00}\right)=\sum_{n \in N, b \in B}[\alpha \cdot \pi](n \omega b) F_{(0,0 ; \phi)}\left(x_{00}\right)+\sum_{b \in B}[\alpha \cdot \pi](b) F_{(0,0 ; \phi)}\left(x_{00}\right) \\
=q^{3}\left[\sum_{n \in N}[\alpha \cdot \pi](n)[\alpha \cdot \pi](\omega) A+A\right]\left(x_{00}\right)=q^{3}\left[q^{3}[\alpha \cdot \pi](\omega) A\left(x_{00}\right)+A\left(x_{00}\right)\right]=q^{3}(q+1)(q-1)\left(q^{2}+q+1\right)^{2} \neq 0 .
\end{gathered}
$$

As $[\alpha \cdot \pi](\omega) A \neq A$, this means that $\operatorname{dim}\left(V_{\alpha}\right)^{G}=1$. So there exists two constants $a, b \in \mathbb{C}^{\times}$such that $a A+b B$ is $[\alpha \cdot \pi](\omega)$-invariant. By the above diagram, we can let $a=q, b=-(q-1)$.

Corollary 4.7. For any character $\chi \in \operatorname{Irr}\left(F^{\times}\right)$and $\alpha^{-1}=\chi^{-1} \circ \mathrm{N}_{K / F}$, we have:

(1) $\operatorname{dim}_{\mathbb{C}} \operatorname{Hom}_{G}\left(V, \alpha^{-1} \cdot 1_{G}\right)=1$;

(2) $\operatorname{dim}_{\mathbb{C}} \operatorname{Hom}_{G}\left(V, \alpha^{-1} \cdot \mathrm{St}_{G}\right)=1$.

Proof. 1) $\operatorname{Hom}_{\mathbb{C}}\left(V, \alpha^{-1} \cdot 1_{G}\right) \simeq\left(V_{\alpha}\right)^{G}$, which is of dimension smaller than 2. As $\alpha \cdot \pi(\omega) A \neq A$ and $\alpha \cdot \pi(\omega)(q A-$ $(q-1) B)=q A-(q-1) B$, we know that $\operatorname{dim}_{\mathbb{C}}\left(V_{\alpha}\right)^{G}=1$.

2 ) It follows from the above (1) and Proposition 4.4 . 
Proposition 4.8. The non-cuspidal part of the Weil representation $\pi$ is presented as follows:

$$
\pi_{\text {non-cusp }} \simeq \bigoplus_{\sigma \in \operatorname{Irr}_{\text {non-cusp }}\left(\mathrm{GL}_{2}(F)\right)} \mathrm{Bc}_{K / F}(\sigma),
$$

where $\pi_{n o n-c u s p}$ is the non-cuspidal part of the representation $\pi$ and $\mathrm{Bc}_{K / F}$ is the map of base change from $\operatorname{Irr}\left(\mathrm{GL}_{2}(F)\right)$ to $\operatorname{Irr}\left(\mathrm{GL}_{2}(K)\right)$.

Proof. It follows from Theorem 1.5 (2), Proposition 4.4 and Corollary 4.7

Corollary 4.9. The total dimension of the cuspidal part of $\pi$ equals $\frac{(q-1) q\left(q^{3}-1\right)}{2}$.

Proof. By Proposition 4.8, the dimension of the non-cuspidal part of $\pi$ equals

$$
\left(q^{3}+1\right) \cdot \frac{(q-1)(q-2)}{2}+1 \cdot(q-1)+q^{3} \cdot(q-1)=\frac{(q-1) q\left(q^{3}+1\right)}{2} ;
$$

the dimension of $\pi$ is $(q-1) q^{4}$, and $(q-1) q^{4}-\frac{(q-1) q\left(q^{3}+1\right)}{2}=\frac{(q-1) q\left(q^{3}-1\right)}{2}$.

4.7. We continue the above discussion and determine the cuspidal part of $\pi$.

Now let $K_{1}$ (resp. $F_{1}$ ) be a quadratic field extension of $K$ (resp. $F$ ). Assume $K_{1} \supset F_{1}$. Let $\rho\left(\right.$ resp. $\left.\rho_{1}\right)$ denote the Weil representation of $\mathbf{G S p}_{W_{0}}(F)\left(\right.$ resp. $\left.\mathbf{G S p}_{W_{0}}\left(F_{1}\right)\right)$. Denote by $\pi=\left.\rho\right|_{\mathrm{GL}_{2}(K)}$ and $\pi_{1}=\left.\rho_{1}\right|_{\mathrm{GL}_{2}\left(K_{1}\right)}$. By Proposition 1.8 in Section 1.5 there exists a unique representation $\widetilde{\rho_{1}}$ of the group $\mathbf{G S p}_{W_{0}}\left(F_{1}\right) \rtimes \operatorname{Gal}\left(F_{1} / F\right)$ such that $0-$ $\operatorname{res}\left(\widetilde{\rho_{1}}\right)=\rho_{1}$, and $1-\operatorname{res}\left(\widetilde{\rho_{1}}\right)=\rho$. By the result in Section 4.2, there exists a morphism from $\operatorname{Res}_{K / F}\left(\mathbf{G L}_{2}\right)$ to $\mathbf{G S p}_{W_{0}}$, which induces a map $\widetilde{p_{1}}: \mathbf{G L}_{2}\left(K_{1}\right) \rtimes \operatorname{Gal}\left(K_{1} / K\right) \simeq \operatorname{Res}_{K / F}\left(\mathbf{G L}_{2}\right)\left(F_{1}\right) \rtimes \operatorname{Gal}\left(F_{1} / F\right) \longrightarrow \mathbf{G S p}_{W_{0}}\left(F_{1}\right) \rtimes \operatorname{Gal}\left(F_{1} / F\right)$.

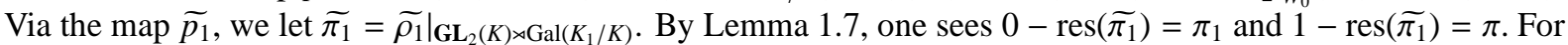
a cuspidal representation $\Pi_{\Lambda}$ of $\mathrm{GL}_{2}(K)$, by Theorem 1.5 we know $\mathrm{Bc}_{K_{1} / K}\left(\Pi_{\Lambda}\right)=\Pi_{\Lambda, \Lambda^{q^{3}}}$. Let $\Pi_{\Lambda, \Lambda^{q^{3}}}$ denote the unique representation of the group $\mathbf{G L}_{2}\left(K_{1}\right) \rtimes \operatorname{Gal}\left(K_{1} / K\right)$ such that $0-\operatorname{res}\left(\widetilde{\Pi_{\Lambda, \Lambda} q^{3}}\right)=\Pi_{\Lambda, \Lambda q^{3}}$ and $1-\operatorname{res}\left(\widetilde{\Pi_{\Lambda, \Lambda} q^{3}}\right)=$ $\Pi_{\Lambda}$. By Proposition 4.4, $\left\langle\pi_{1}, \Pi_{\Lambda, \Lambda^{q^{3}}}\right\rangle_{\mathrm{GL}_{2}\left(K_{1}\right)}=1$ for $\Lambda=\lambda \circ \mathrm{N}_{K_{1} / F_{1}}$, where $\lambda$ is a regular character of $F_{1}^{\times}$. By Lemma 1.6 (i), we have

$$
\begin{aligned}
& \left\langle\operatorname{Tr} \widetilde{\pi_{1}}, \operatorname{Tr} \widetilde{\Pi_{\Lambda, \Lambda} q^{3}}\right\rangle_{\mathbf{G L}_{2}\left(K_{1}\right) \rtimes \operatorname{Gal}\left(K_{1} / K\right)} \\
& =\frac{1}{\left|\mathrm{GL}_{2}\left(K_{1}\right) \rtimes \operatorname{Gal}(K / F)\right|}\left(\sum_{g \in \mathrm{GL}_{2}\left(K_{1}\right)} \operatorname{Tr} \widetilde{\pi_{1}}((1, g)) \overline{\operatorname{Tr} \widetilde{\Pi_{\Lambda, \Lambda} q^{3}}((1, g))}+\sum_{g \in \mathrm{GL}_{2}\left(K_{1}\right)} \operatorname{Tr} \widetilde{\pi_{1}}((\sigma, g)) \overline{\operatorname{Tr} \widetilde{\Pi_{\Lambda, \Lambda} q^{3}}((\sigma, g))}\right) \\
& =\frac{\left|\mathrm{GL}_{2}\left(K_{1}\right)\right|}{\left|\mathrm{GL}_{2}\left(K_{1}\right) \rtimes \mathrm{Gal}\left(K_{1} / K\right)\right|}\left\langle\operatorname{Tr} \pi_{1}, \operatorname{Tr} \Pi_{\Lambda, \Lambda^{q^{3}}}\right\rangle_{\mathrm{GL}_{2}\left(K_{1}\right)}+\frac{\left|\mathrm{GL}_{2}\left(K_{1}\right)\right|}{\left|\mathrm{GL}_{2}\left(K_{1}\right) \rtimes \operatorname{Gal}\left(K_{1} / K\right)\right|}\left\langle\operatorname{Tr} \pi, \operatorname{Tr} \Pi_{\Lambda}\right\rangle_{\mathrm{GL}_{2}(K)} \\
& =\frac{1}{2}\left(\left\langle\operatorname{Tr} \pi_{1}, \operatorname{Tr} \Pi_{\Lambda, \Lambda^{q^{3}}}\right\rangle_{\mathrm{GL}_{2}\left(K_{1}\right)}+\left\langle\operatorname{Tr} \pi, \operatorname{Tr} \Pi_{\Lambda}\right\rangle_{\mathrm{GL}_{2}(K)}\right)=\frac{1}{2}\left(1+\left\langle\operatorname{Tr} \pi, \operatorname{Tr} \Pi_{\Lambda}\right\rangle_{\mathrm{GL}_{2}(K)}\right)
\end{aligned}
$$

for $\Lambda=\lambda \circ \mathrm{N}_{K_{1} / F_{1}}$. It follows that for such $\Lambda,\left\langle\pi, \Pi_{\Lambda}\right\rangle_{\mathrm{GL}_{2}(K)} \geq 1$. By Corollary 4.9, we see $\left\langle\pi, \Pi_{\Lambda}\right\rangle_{\mathrm{GL}_{2}(K)}=1$ and it will also turn out that there are no other kind of cuspidal sub-representations of $\pi$. Finally we achieve the main theorem in this section:

4.8.

Theorem 4.10. The representation $(\pi, V)$ has the following decomposition:

$$
\pi \simeq \bigoplus_{\sigma \in \operatorname{Irr}\left(\mathrm{GL}_{2}(F)\right)} \mathrm{Bc}_{K / F}(\sigma)
$$

where $\operatorname{Irr}\left(\mathrm{GL}_{2}(F)\right)$ is the set of the classes of the irreducible representations of $\mathrm{GL}_{2}(F)$, and $B c_{K / F}$ is the base change from $\operatorname{Irr}\left(\mathrm{GL}_{2}(F)\right)$ to $\operatorname{Irr}\left(\mathrm{GL}_{2}(K)\right)$.

Proof. It follows from Proposition 4.8 for non-cuspidal representations and the above discussion for cuspidal representations. 
4.9. Appendix 1. In the following, we explain how to get the formulas in Proposition 4.2.

(1):

$$
\begin{aligned}
& {[\pi(u(b) F)]\left(\left(\begin{array}{ll}
0 & 0 \\
\beta & y
\end{array}\right), \psi\right)} \\
& =[\rho(i(u(b)) F)]\left(\left(\begin{array}{ll}
0 & 0 \\
\beta & y
\end{array}\right), \psi\right) \\
& =\left[\rho\left(\left(\begin{array}{cc}
m & 0 \\
0 & m^{\vee}
\end{array}\right)\left(\begin{array}{cc}
1 & m^{-1} n \\
0 & 1
\end{array}\right)\right) F\right]\left(\left(\begin{array}{cc}
0 & 0 \\
\beta & y
\end{array}\right), \psi\right) \\
& =\chi_{q}^{+}\left(\operatorname{det}_{X_{0}} m\right)\left[\rho\left(\left(\begin{array}{cc}
1 & m^{-1} n \\
0 & 1
\end{array}\right)\right) F\right]\left(m^{\vee-1}\left(\begin{array}{cc}
0 & 0 \\
\beta & y
\end{array}\right), \psi\right) \\
& =\left[\rho\left(\left(\begin{array}{cc}
1 & m^{-1} n \\
0 & 1
\end{array}\right)\right) F\right]\left(\left(\begin{array}{cc}
0 & 0 \\
\beta-b^{\sigma^{2}} y & y
\end{array}\right), \psi\right) \\
& =\psi\left(\frac{1}{2}\left\langle m^{-1} n\left(\begin{array}{cc}
0 & 0 \\
\beta-b^{\sigma^{2}} y & y
\end{array}\right), \quad\left(\begin{array}{cc}
0 & 0 \\
\beta-b^{\sigma^{2}} y & y
\end{array}\right)\right\rangle\right) F\left(\left(\begin{array}{cc}
0 & 0 \\
\beta-b^{\sigma} y & y
\end{array}\right), \psi\right) \\
& =\psi\left(\frac{1}{2}\left\langle m^{-1}\left(\begin{array}{cc}
\operatorname{Tr}_{E / F}\left(b b^{\sigma} \beta\right)-2 \mathrm{~N}_{K / F}(b) y & b^{\sigma} \beta^{\sigma}+b \beta^{\sigma^{2}}-b b^{\sigma} y \\
0 & 0
\end{array}\right), \quad\left(\begin{array}{cc}
0 & 0 \\
\beta-b^{\sigma^{2}} y & y
\end{array}\right)\right\rangle\right) F\left(\left(\begin{array}{cc}
0 & 0 \\
\beta-b^{\sigma^{2}} y & y
\end{array}\right), \psi\right) \\
& =\psi\left(\frac{1}{2}\left\langle\left(\begin{array}{cc}
\mathrm{N}_{K / F}(b) y-\operatorname{Tr}_{K / F}\left(b b^{\sigma} \beta\right) & b^{\sigma} \beta^{\sigma}+b \beta^{\sigma^{2}}-b b^{\sigma} y \\
0 & 0
\end{array}\right), \quad\left(\begin{array}{cc}
0 & 0 \\
\beta-b^{\sigma^{2}} y & y
\end{array}\right)\right\rangle\right) F\left(\left(\begin{array}{cc}
0 & 0 \\
\beta-b^{\sigma^{2}} y & y
\end{array}\right), \psi\right) \\
& =\psi\left(\operatorname{Tr}_{K / F}\left(b b^{\sigma} \beta y\right)-\mathrm{N}_{K / F}(b) y^{2}-\operatorname{Tr}_{K / F}\left(b \beta \beta^{\sigma^{2}}\right)\right) F\left(\left(\begin{array}{cc}
0 & 0 \\
\beta-b^{\sigma^{2}} y & y
\end{array}\right), \psi\right) \text {. }
\end{aligned}
$$

(2):

$$
\begin{aligned}
& {[\pi(h(a, d)) F]\left(\left(\begin{array}{ll}
0 & 0 \\
\beta & y
\end{array}\right), \psi\right)} \\
& =[\rho(i(h(a, d))) F]\left(\left(\begin{array}{ll}
0 & 0 \\
\beta & y
\end{array}\right), \psi\right) \\
& =\left[\rho\left(\left(\begin{array}{cc}
m & 0 \\
0 & n
\end{array}\right)\left(\begin{array}{cc}
1 & 0 \\
0 & \mathrm{~N}_{K / F}(a d)^{-1}
\end{array}\right)\left(\begin{array}{cc}
1 & 0 \\
0 & \mathrm{~N}_{K / F}(a d)
\end{array}\right)\right) F\right]\left(\left(\begin{array}{ll}
0 & 0 \\
\beta & y
\end{array}\right), \psi\right) \\
& =\chi_{q}^{+}\left(\operatorname{det}_{X_{0}}(m)\right)\left[\rho\left(\left(\begin{array}{cc}
1 & 0 \\
0 & \mathrm{~N}_{K / F}(a d)
\end{array}\right)\right) F\right]\left(n^{-1}\left(\begin{array}{cc}
0 & 0 \\
\mathrm{~N}_{K / F}(a d) \beta & \mathrm{N}_{K / F}(a d) y
\end{array}\right), \psi\right) \\
& =\chi_{q}^{+}\left(\mathrm{N}_{K / F}(a d)\right)\left[\rho\left(\left(\begin{array}{cc}
1 & 0 \\
0 & \mathrm{~N}_{K / F}(a d)
\end{array}\right)\right) F\right]\left(\left(\begin{array}{cc}
0 & 0 \\
\frac{\mathrm{N}_{K / F}(a d)}{d d^{\sigma} a^{\sigma^{2}}} \beta & \mathrm{N}_{K / F}(a) y
\end{array}\right), \psi\right) \\
& =\chi_{q}^{+}\left(\mathrm{N}_{K / F}(a d)\right) F\left(\left(\begin{array}{cc}
0 & 0 \\
\frac{\mathrm{N}_{K / F}(a d)}{d d^{\sigma} a^{\sigma^{2}}} \beta & \mathrm{N}_{K / F}(a) y
\end{array}\right), \psi^{\mathrm{N}_{K / F}(a d)^{-1}}\right) \text {. }
\end{aligned}
$$

(3): Assume $K=F(\xi)$. For a matrix $X$, we denote its transpose by ${ }^{t} X$. Choose a basis $\mathcal{A}=\left\{m_{0}=e_{1} \otimes e_{1} \otimes e_{1}, m_{1}=\right.$ $\xi e_{1} \otimes e_{1} \otimes e_{2}+\xi^{\sigma} e_{2} \otimes e_{1} \otimes e_{1}+\xi^{\sigma^{2}} e_{1} \otimes e_{2} \otimes e_{1}, m_{2}=\xi^{\sigma} e_{1} \otimes e_{1} \otimes e_{2}+\xi^{\sigma^{2}} e_{2} \otimes e_{1} \otimes e_{1}+\xi e_{1} \otimes e_{2} \otimes e_{1}, m_{3}=$ $\xi^{\sigma^{2}} e_{1} \otimes e_{1} \otimes e_{2}+\xi e_{2} \otimes e_{1} \otimes e_{1}+\xi^{\sigma} e_{1} \otimes e_{2} \otimes e_{1} ; n_{0}=-e_{2} \otimes e_{2} \otimes e_{2}, n_{1}=\xi e_{2} \otimes e_{2} \otimes e_{1}+\xi^{\sigma} e_{1} \otimes e_{2} \otimes e_{2}+\xi^{\sigma^{2}} e_{2} \otimes e_{1} \otimes e_{2}, n_{2}=$ $\left.\xi^{\sigma} e_{2} \otimes e_{2} \otimes e_{1}+\xi^{\sigma^{2}} e_{1} \otimes e_{2} \otimes e_{2}+\xi e_{2} \otimes e_{1} \otimes e_{2}, n_{3}=\xi^{\sigma^{2}} e_{2} \otimes e_{2} \otimes e_{1}+\xi e_{1} \otimes e_{2} \otimes e_{2}+\xi^{\sigma} e_{2} \otimes e_{1} \otimes e_{2}\right\}$ in $W_{0}$. Then by Corollary 4.1 (3), we know $i(\omega)\left(m_{i}\right)=n_{i}$ and $i(\omega)\left(n_{i}\right)=-m_{i}$ for $0 \leq i \leq 3$. By calculation, we obtain

$$
\left(\begin{array}{cccccc}
\left\langle m_{0}, m_{0}\right\rangle & \ldots & \left\langle m_{0}, m_{3}\right\rangle & \left\langle m_{0}, n_{0}\right\rangle & \ldots & \left\langle m_{0}, n_{3}\right\rangle \\
\vdots & \ddots & \vdots & \vdots & \ddots & \vdots \\
\left\langle m_{3}, m_{0}\right\rangle & \ldots & \left\langle m_{3}, m_{3}\right\rangle & \left\langle m_{3}, n_{0}\right\rangle & \ldots & \left\langle m_{3}, n_{3}\right\rangle \\
\left\langle n_{0}, m_{0}\right\rangle & \ldots & \left\langle n_{0}, m_{3}\right\rangle & \left\langle n_{0}, n_{0}\right\rangle & \ldots & \left\langle n_{0}, n_{3}\right\rangle \\
\vdots & \ddots & \vdots & \vdots & \ddots & \vdots \\
\left\langle n_{3}, m_{0}\right\rangle & \ldots & \left\langle n_{3}, m_{3}\right\rangle & \left\langle n_{3}, n_{0}\right\rangle & \ldots & \left\langle n_{3}, n_{3}\right\rangle
\end{array}\right)=\left(\begin{array}{cc}
0 & A \\
-A & 0
\end{array}\right)
$$


where $A=\left(\begin{array}{cccc}-1 & 0 & 0 & 0 \\ 0 & -\operatorname{Tr}_{K / F}\left(\xi^{2}\right) & -\operatorname{Tr}_{K / F}\left(\xi \xi^{\sigma}\right) & -\operatorname{Tr}_{K / F}\left(\xi \xi^{\sigma}\right) \\ 0 & -\operatorname{Tr}_{K / F}\left(\xi \xi^{\sigma}\right) & -\operatorname{Tr}_{K / F}\left(\xi^{2}\right) & -\operatorname{Tr}_{K / F}\left(\xi \xi^{\sigma}\right) \\ 0 & -\operatorname{Tr}_{K / F}\left(\xi \xi^{\sigma}\right) & -\operatorname{Tr}_{K / F}\left(\xi \xi^{\sigma}\right) & -\operatorname{Tr}_{K / F}\left(\xi^{2}\right)\end{array}\right)$.

Suppose $A={ }^{t} P_{1} P_{1}$ and $\left(g_{0}, \cdots, g_{3} ; h_{0}, \cdots, h_{3}\right)=\left(m_{0}, \cdots, m_{3} ; n_{0}, \cdots, n_{3}\right) P$ for some $P=\left(\begin{array}{cc}P_{1}^{-1} & 0 \\ 0 & P_{1}^{-1}\end{array}\right)$. Then:

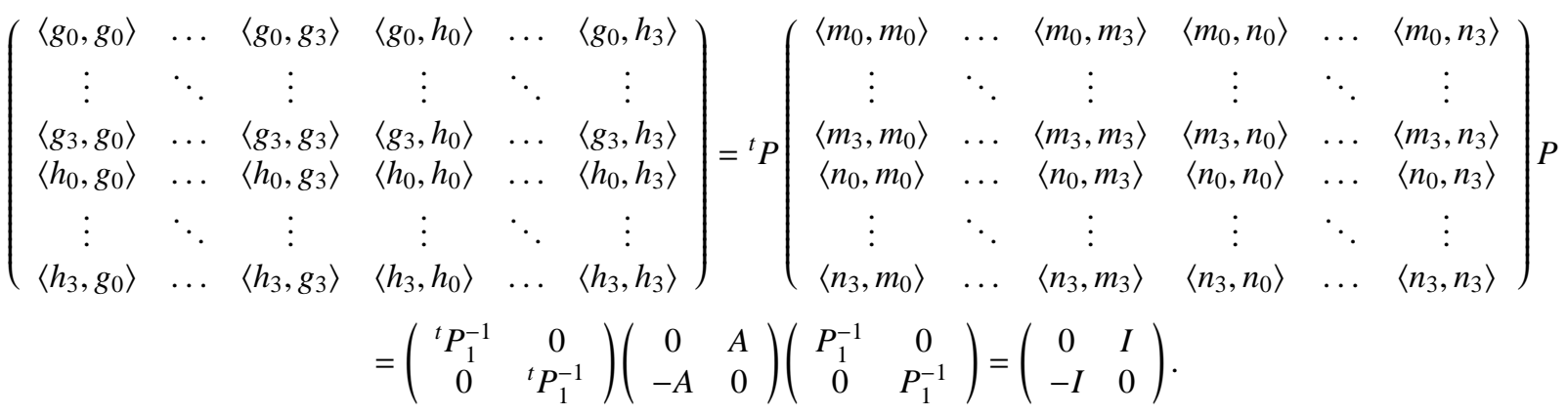
i.e. the set $\left\{g_{0}, \cdots, g_{3} ; h_{0}, \cdots, h_{3}\right\}$ is a symplectic basis of $W_{0}$. Moreover $i(\omega)\left(g_{0}, \cdots, g_{3} ; h_{0}, \cdots, h_{3}\right)=$ $\left(g_{0}, \cdots, g_{3} ; h_{0}, \cdots, h_{3}\right) P^{-1}\left(\begin{array}{cc}0 & -I \\ I & 0\end{array}\right) P$. And $P^{-1}\left(\begin{array}{cc}0 & -I \\ I & 0\end{array}\right) P=\left(\begin{array}{cc}0 & -I \\ I & 0\end{array}\right)=\omega_{\mathrm{GSp}\left(W_{0}\right)}^{-1} \in \mathrm{GSp}\left(W_{0}\right)$ with respect to the symplectic basis $\left\{g_{0}, \cdots, g_{3} ; h_{0}, \cdots, h_{3}\right\}$. Now let $\alpha=a_{1} \xi+a_{2} \xi^{\sigma}+a_{3} \xi^{\sigma^{2}}, \beta=b_{1} \xi+b_{2} \xi^{\sigma}+b_{3} \xi^{\sigma^{2}} \in K$. Put $b=\left(b_{0}, \cdots, b_{3}\right)$ and $a=\left(a_{0}, \cdots, a_{3}\right)$. Then

$$
\begin{gathered}
{[\pi(\omega) F]\left(\left(\begin{array}{cc}
0 & 0 \\
\beta & -b_{0}
\end{array}\right), \psi\right)=\rho[i(\omega) F]\left(\left(n_{0}, \cdots, n_{3}\right)^{t} b, \psi\right)} \\
=q^{-2} \sum_{\left(n_{0}, \cdots, n_{3}\right)^{t} a \in Y_{0}} F\left(\left(n_{0}, \cdots, n_{3}\right)^{t} a, \psi\right) \psi\left(\left\langle\left(n_{0}, \cdots, n_{3}\right)^{t} a, \omega_{\mathrm{GSp}\left(W_{0}\right)}\left(\left(n_{0}, \cdots, n_{3}\right)^{t} b\right)\right\rangle\right) \\
=q^{-2} \sum_{\left(_{0}, \cdots, n_{3}\right)^{t} a \in Y_{0}} F\left(\left(n_{0}, \cdots, n_{3}\right)^{t} a, \psi\right) \psi\left(\left\langle\left(n_{0}, \cdots, n_{3}\right)^{t} a, i\left(\omega^{-1}\right)\left[\left(n_{0}, \cdots, n_{3}\right)^{t} b\right]\right\rangle\right) \\
=q^{-2} \sum_{\left(n_{0}, \cdots, n_{3}\right)^{t} a \in Y_{0}} F\left(-a_{0} e_{2} \otimes e_{2} \otimes e_{2}+\alpha e_{2} \otimes e_{2} \otimes e_{1}+\alpha^{\sigma} e_{1} \otimes e_{2} \otimes e_{2}+\alpha^{\sigma^{2}} e_{2} \otimes e_{1} \otimes e_{2}, \psi\right) \\
\psi\left(\left\langle-a_{0} e_{2} \otimes e_{2} \otimes e_{2}+\alpha e_{2} \otimes e_{2} \otimes e_{1}+\alpha^{\sigma} e_{1} \otimes e_{2} \otimes e_{2}+\alpha^{\sigma^{2}} e_{2} \otimes e_{1} \otimes e_{2},\right.\right. \\
\left.\left.b_{0} e_{1} \otimes e_{1} \otimes e_{1}+\beta e_{1} \otimes e_{1} \otimes e_{2}+\beta^{\sigma} e_{2} \otimes e_{1} \otimes e_{1}+\beta^{\sigma^{2}} e_{1} \otimes e_{2} \otimes e_{1}\right\rangle\right) \\
\sum_{a_{0} \in F, \alpha \in K} F\left(-a_{0} e_{2} \otimes e_{2} \otimes e_{2}+\alpha e_{2} \otimes e_{2} \otimes e_{1}+\alpha^{\sigma} e_{1} \otimes e_{2} \otimes e_{2}+\alpha^{\sigma^{2}} e_{2} \otimes e_{1} \otimes e_{2}, \psi\right) \psi\left(a_{0} b_{0}+\operatorname{Tr}_{K / F}(\alpha \beta)\right) \\
=q^{-2} \sum_{a_{0} \in F, \alpha \in K} F\left(\left(\begin{array}{cc}
0 & 0 \\
\alpha & -a_{0}
\end{array}\right), \psi\right) \psi\left(a_{0} b_{0}+\operatorname{Tr}_{K / F}(\alpha \beta)\right) .
\end{gathered}
$$

Finally, we obtain

$$
[\pi(\omega) F]\left(\left(\begin{array}{ll}
0 & 0 \\
\beta & y
\end{array}\right), \psi\right)=q^{-2} \sum_{\beta^{\prime} \in K, y^{\prime} \in F} F\left(\left(\begin{array}{cc}
0 & 0 \\
\beta^{\prime} & y^{\prime}
\end{array}\right), \psi\right) \psi\left(y y^{\prime}+\operatorname{Tr}_{K / F}\left(\beta \beta^{\prime}\right)\right) .
$$

4.10. Appendix 2. We put the calculations for the table in Section 4.6 in this appendix. From the definition, we see:

$$
\begin{gathered}
A\left(\left(\begin{array}{ll}
0 & 0 \\
\beta & y
\end{array}\right), \phi^{k}\right)=\sum_{t \in T}[\alpha \cdot \pi](t) F_{(0,0 ; \phi)}\left(\left(\begin{array}{ll}
0 & 0 \\
\beta & y
\end{array}\right), \phi^{k}\right) \\
=\chi \chi_{q}^{+}\left(\mathrm{N}_{K / F}(a d)\right) \sum_{a, d \in K^{\times}} F_{(0,0 ; \phi)}\left(\left(\begin{array}{cc}
0 & 0 \\
\frac{\mathrm{N}_{K / F}(a d)}{d d^{\sigma} a^{\sigma^{2}}} \beta & \mathrm{N}_{K / F}(a) y
\end{array}\right), \phi^{\mathrm{N}_{K / F}(a d)^{-1} k}\right) \\
=\sum_{a, d \in K^{\times}, \mathrm{N}_{K / F}(a d)=k} \chi \chi_{q}^{+}(k) F_{(0,0 ; \phi)}\left(\left(\begin{array}{cc}
0 & 0 \\
\frac{\mathrm{N}_{K / F}(a d)}{d d^{\sigma} a^{\sigma^{2}}} \beta & \mathrm{N}_{K / F}(a) y
\end{array}\right), \phi\right) ;
\end{gathered}
$$


ON A QUESTION OF DRINFELD ON THE WELL REPRESENTATION: THE FINITE FIELD CASE

27

$$
\begin{aligned}
& B\left(\left(\begin{array}{ll}
0 & 0 \\
\beta & y
\end{array}\right), \phi^{k}\right)=\sum_{t \in T}[\alpha \cdot \pi](t) G_{(0,1 ; \phi)}\left(\left(\begin{array}{ll}
0 & 0 \\
\beta & y
\end{array}\right), \phi^{k}\right) \\
& =\sum_{a, d \in K^{\times}} \chi \chi_{q}^{+}\left(\mathrm{N}_{K / F}(a d)\right) G_{(0,1 ; \phi)}\left(\left(\begin{array}{cc}
0 & 0 \\
\frac{\mathrm{N}_{K / F}(a d)}{d d^{\sigma} a^{\sigma^{2}}} \beta & \mathrm{N}_{K / F}(a) y
\end{array}\right), \phi^{\mathrm{N}_{K / F}(a d)^{-1} k}\right) \\
& =\sum_{a, d \in K^{\times}, \mathrm{N}_{K / F}(a d)=k} \chi \chi_{q}^{+}(k) G_{(0,1 ; \phi)}\left(\left(\begin{array}{cc}
0 & 0 \\
\frac{\mathrm{N}_{K / F}(a d)}{d d^{\sigma} a^{\sigma^{2}}} \beta & \mathrm{N}_{K / F}(a) y
\end{array}\right), \phi\right) ; \\
& ([\alpha \cdot \pi](\omega) A)\left(\left(\begin{array}{ll}
0 & 0 \\
\beta & y
\end{array}\right), \phi^{k}\right) \\
& =\sum_{t \in T}[\alpha \cdot \pi](t)\left([\alpha \cdot \pi](\omega) F_{(0,0 ; \phi)}\right)\left(\left(\begin{array}{ll}
0 & 0 \\
\beta & y
\end{array}\right), \phi^{k}\right) \\
& =\sum_{a, d \in K^{\times}} \chi \chi_{q}^{+}\left(\mathrm{N}_{K / F}(a d)\right)\left([\alpha \cdot \pi](\omega) F_{(0,0 ; \phi)}\right)\left(\left(\begin{array}{cc}
0 & 0 \\
\frac{\mathrm{N}_{K / F}(a d)}{d d^{\sigma} a^{\sigma^{2}}} \beta & \mathrm{N}_{K / F}(a) y
\end{array}\right), \phi^{k \mathrm{~N}_{K / F}(a d)^{-1}}\right) \\
& =q^{-2} \sum_{a, d \in K^{\times}} \chi \chi_{q}^{+}\left(\mathrm{N}_{K / F}(a d)\right) \sum_{\left(\begin{array}{cc}
0 & 0 \\
\beta^{\prime} & y^{\prime}
\end{array}\right) \in Y_{0}} F_{(0,0 ; \phi)}\left(\left(\begin{array}{cc}
0 & 0 \\
\beta^{\prime} & y^{\prime}
\end{array}\right), \phi^{k \mathrm{~N}_{K / F}(a d)^{-1}}\right) \phi^{k \mathrm{~N}_{K / F}(a d)^{-1}}\left(\mathrm{~N}_{K / F}(a) y y^{\prime}+\operatorname{Tr}_{K / F}\left(\frac{\mathrm{N}_{K / F}(a d)}{d d^{\sigma} a^{\sigma^{2}}} \beta \beta^{\prime}\right)\right) \\
& =q^{-2} \sum_{a, d \in K^{\times}, \mathrm{N}_{K / F}(a d)=k} \chi \chi_{q}^{+}(k)=q^{-2} \chi \chi_{q}^{+}(k)\left(q^{3}-1\right)\left(q^{2}+q+1\right) ;
\end{aligned}
$$

Notice: $\left.G_{(0,1 ; \phi)}\left(\begin{array}{ll}0 & 0 \\ \beta & 1\end{array}\right), \phi\right)=\phi\left(-\mathrm{N}_{K / F}(\beta)\right)$ by the formula 4.1).

$$
\begin{aligned}
& \left.(\alpha \cdot \pi(\omega) B)\left(\left(\begin{array}{cc}
0 & 0 \\
\beta & y
\end{array}\right), \phi^{k}\right)=\sum_{t \in T} \alpha \cdot \pi(t)[\alpha \cdot \pi(\omega)] G_{(0,1 ; \phi)}\left(\begin{array}{ll}
0 & 0 \\
\beta & y
\end{array}\right), \phi^{k}\right) \\
& =\sum_{a, d \in K^{\times}} \chi \chi_{q}^{+}\left(\mathrm{N}_{K / F}(a d)\right)[\alpha \cdot \pi](\omega) G_{(0,1 ; \phi)}\left(\left(\begin{array}{cc}
0 & 0 \\
\frac{\mathrm{N}_{K / F}(a d)}{d d^{\sigma} a^{\sigma^{2}}} \beta & \mathrm{N}_{K / F}(a) y
\end{array}\right), \phi^{k \mathrm{~N}_{K / F}(a d)^{-1}}\right) \\
& =q^{-2} \sum_{a, d \in K^{\times}} \chi \chi_{q}^{+}\left(\mathrm{N}_{K / F}(a d)\right) \sum_{\left(\begin{array}{cc}
0 & 0 \\
\beta^{\prime} & y^{\prime}
\end{array}\right) \in Y_{0}} G_{(0,1 ; \phi)}\left(\left(\begin{array}{cc}
0 & 0 \\
\beta^{\prime} & y^{\prime}
\end{array}\right), \phi^{k \mathrm{~N}_{K / F}(a d)^{-1}}\right) \phi^{k \mathrm{~N}_{K / F}(a d)^{-1}}\left(\mathrm{~N}_{K / F}(a) y y^{\prime}+\operatorname{Tr}_{K / F}\left(\frac{\mathrm{N}_{K / F}(a d)}{d d^{\sigma} a^{\sigma^{2}}} \beta \beta^{\prime}\right)\right) \\
& =q^{-2} \sum_{a, d \in K^{\times}, \mathrm{N}_{K / F}(a d)=k} \chi \chi_{q}^{+}(k) \sum_{\beta^{\prime} \in K} \phi\left(-\mathrm{N}_{K / F}\left(\beta^{\prime}\right)\right) \phi\left(\mathrm{N}_{K / F}(a) y+\operatorname{Tr}_{K / F}\left(a a^{\sigma} d^{\sigma^{2}} \beta \beta^{\prime}\right)\right) \text {; }
\end{aligned}
$$

$$
\begin{gathered}
\left.A\left(x_{00}\right)=\sum_{a, d \in K^{\times}, \mathrm{N}_{K / F}(a d)=1} F_{(0,0, \phi)}\left(\begin{array}{ll}
0 & 0 \\
0 & 0
\end{array}\right), \phi\right) \\
=\sum_{a, d \in K^{\times}, \mathrm{N}_{K / F}(a d)=1} 1=\left(q^{3}-1\right)\left(q^{2}+q+1\right) . \\
A\left(x_{10}\right)=A\left(x_{01}\right)=A\left(y_{k}\right)=0 .
\end{gathered}
$$

(2)

$$
\begin{gathered}
B\left(x_{00}\right)=\sum_{a, d \in K^{\times}, \mathrm{N}_{K / F}(a d)=1} G_{(0,1 ; \phi)}\left(\left(\begin{array}{ll}
0 & 0 \\
0 & 0
\end{array}\right), \phi\right)=0=B\left(x_{10}\right) . \\
B\left(x_{01}\right)=\sum_{a, d \in K^{\times}, \mathrm{N}_{K / F}(a d)=1} G_{(0,1 ; \phi)}\left(\left(\begin{array}{cc}
0 & 0 \\
0 & \mathrm{~N}_{K / F}(a)
\end{array}\right), \phi\right)
\end{gathered}
$$




$$
\begin{gathered}
=\sum_{a, d \in K^{\times}, \mathrm{N}_{K / F}(a)=\mathrm{N}_{K / F}(d)=1} 1=\left(q^{2}+q+1\right)^{2} . \\
B\left(y_{k}\right)=\sum_{t \in T}[\alpha \cdot \pi](t) G_{(0,1, \phi)}\left(y_{k}\right) \\
=\sum_{a, d \in K^{\times}, \mathrm{N}_{K / F}(a d)=k} \chi \chi_{q}^{+}(k) G_{(0,1 ; \phi)}\left(\left(\begin{array}{cc}
0 & 0 \\
\frac{\mathrm{N}_{K / F}(a d)}{d d^{\sigma} a^{\sigma^{2}}} & \mathrm{~N}_{K / F}(a)
\end{array}\right), \phi\right) \\
=\sum_{a, d \in K^{\times}, \mathrm{N}_{K / F}(a)=1, \mathrm{~N}_{K / F}(d)=k} \chi \chi_{q}^{+}(k) G_{(0,1 ; \phi)}\left(\left(\begin{array}{cc}
0 & 0 \\
a a^{\sigma} d^{\sigma^{2}} & 1
\end{array}\right), \phi\right) \\
\sum_{a, d \in K^{\times}, \mathrm{N}_{K / F}(a)=1, \mathrm{~N}_{K / F}(d)=k} \chi \chi_{q}^{+}(k) \phi\left(-\mathrm{N}_{K / F}\left(a a^{\sigma} d^{\sigma^{2}}\right)\right) \\
\sum_{a, d \in K^{\times}, \mathrm{N}_{K / F}(a)=1, \mathrm{~N}_{K / F}(d)=k} \chi \chi_{q}^{+}(k) \phi(-k) \\
=\phi(-k) \chi \chi_{q}^{+}(k)\left(q^{2}+q+1\right)^{2} .
\end{gathered}
$$

(4)

$$
\begin{aligned}
& {[\alpha \cdot \pi](\omega) A\left(x_{00}\right)=[\alpha \cdot \pi](\omega) A\left(X_{10}\right)=[\alpha \cdot \pi](\omega) A\left(x_{01}\right)=q^{-2}\left(q^{3}-1\right)\left(q^{2}+q+1\right) .} \\
& {[\alpha \cdot \pi](\omega) A\left(y_{k}\right)=\chi \chi_{q}^{+}(k) q^{-2}\left(q^{3}-1\right)\left(q^{2}+q+1\right)=\chi \chi_{q}^{+}(k) q^{-2}(q-1)\left(q^{2}+q+1\right)^{2} .}
\end{aligned}
$$

$\alpha \cdot \pi(\omega) B\left(x_{00}\right)=q^{-2} \sum_{a, d \in K^{\times}, \mathrm{N}_{K / F}(a d)=1, \beta^{\prime} \in K} \phi\left(-\mathrm{N}_{K / F}\left(\beta^{\prime}\right)\right)=q^{-2}\left(q^{3}-1\right)\left(q^{2}+q+1\right)\left(-q^{2}-q\right)=-q^{-1}(q-1)(q+1)\left(q^{2}+q+1\right)^{2}$.

(Since $\sum_{\beta^{\prime} \neq 0} \phi\left(-\mathrm{N}_{K / F}\left(\beta^{\prime}\right)\right)+q^{2}+q+1=0$, we have $\left.\sum_{\beta^{\prime} \in K} \phi\left(-\mathrm{N}_{K / F}\left(\beta^{\prime}\right)\right)=-q^{2}-q\right)$.

$$
\begin{gathered}
\alpha \cdot \pi(\omega) B\left(x_{10}\right)=q^{-2} \sum_{a, d \in K^{\times}, \mathrm{N}_{K / F}(a d)=1} \sum_{\beta^{\prime} \in K} \phi\left(-\mathrm{N}_{K / F}\left(\beta^{\prime}\right)\right) \phi\left(\operatorname{Tr}_{K / F}\left(\frac{1}{d d^{\sigma} a^{\sigma^{2}}} \beta^{\prime}\right)\right. \\
=q^{-2} \sum_{a, d \in K^{\times}, \mathrm{N}_{K / F}(a d)=1, \beta^{\prime} \in K} \phi\left(-\mathrm{N}_{K / F}\left(d d^{\sigma} a^{\sigma^{2}} \beta^{\prime}\right)\right) \phi\left(\operatorname{Tr}_{K / F}\left(\beta^{\prime}\right)\right) \\
=q^{-2} \sum_{\beta^{\prime} \in K} \sum_{a, d \in K^{\times}, \mathrm{N}_{K / F}(a d)=1} \phi\left(-\mathrm{N}_{K / F}(d) \mathrm{N}_{K / F}\left(\beta^{\prime}\right)\right) \phi\left(\operatorname{Tr}_{K / F}\left(\beta^{\prime}\right)\right) \cdots(\star)
\end{gathered}
$$

(i) If $\beta^{\prime}=0, \sum_{a, d \in K^{\times}, \mathrm{N}_{K / F}(a d)=1} \phi\left(-\mathrm{N}_{K / F}(d) \mathrm{N}_{K / F}\left(\beta^{\prime}\right)+\operatorname{Tr}_{K / F}\left(\beta^{\prime}\right)\right)=\sum_{a, d \in K^{\times}, \mathrm{N}_{K / F}(a d)=1} 1=\left(q^{3}-1\right)\left(q^{2}+q+1\right)$;

(ii) If $\beta^{\prime} \neq 0$,

$$
\begin{gathered}
\sum_{a, d \in K^{\times}, \mathrm{N}_{K / F}(a d)=1} \phi\left(-\mathrm{N}_{K / F}(d) \mathrm{N}_{K / F}\left(\beta^{\prime}\right)\right) \\
=\sum_{l \in K^{\times}, \mathrm{N}_{K / F}(l)=1} \sum_{d \in K^{\times}} \phi\left(-\mathrm{N}_{K / F}(d) \mathrm{N}_{K / F}\left(\beta^{\prime}\right)\right) \\
=\left(q^{2}+q+1\right)\left(-q^{2}-q-1\right)=-\left(q^{2}+q+1\right)^{2},
\end{gathered}
$$

SO

$$
\begin{gathered}
(\star)=q^{-2}\left[\left(q^{3}-1\right)\left(q^{2}+q+1\right)+\sum_{\beta^{\prime} \in K^{\times}}-\left(q^{2}+q+1\right)^{2} \phi\left(\operatorname{Tr}_{K / F}\left(\beta^{\prime}\right)\right)\right] \\
=q^{-2}\left[\left(q^{2}+q+1\right)\left(q^{3}-1\right)+\left(q^{2}+q+1\right)^{2}\right]=q^{-2}\left(q^{2}+q+1\right)^{2} q=q^{-1}\left(q^{2}+q+1\right)^{2} . \\
\alpha \cdot \pi(\omega) B\left(x_{01}\right)=q^{-2} \sum_{a, d \in K^{\times}, \mathrm{N}_{K / F}(a d)=1} \sum_{\beta^{\prime} \in K} \phi\left(-\mathrm{N}_{K / F}\left(\beta^{\prime}\right)\right) \phi\left(\mathrm{N}_{K / F}(a)\right) \\
=q^{-2} \sum_{a, d \in K^{\times}, \mathrm{N}_{K / F}(a d)=1} \phi\left(\mathrm{N}_{K / F}(a)\right)\left(-q^{2}-q\right) \\
=q^{-2}\left(q^{2}+q+1\right)\left(-q^{2}-q-1\right)\left(-q^{2}-q\right)=q^{-1}(q+1)\left(q^{2}+q+1\right)^{2} .
\end{gathered}
$$




\section{REFERENCES}

[1] Jorge Soto-Andrade, Représentations de certains groupes symplectiques, Société Mathématique de France. Bulletin. Mémoire. 55-56 (1978).

[2] Colin J. Bushnell, Guy Henniart, The local langlands conjecture for GL(2), Grundlehren der Mathematischen Wissenschaften 335, Springer-Verlag, Berlin, 2006.

[3] François Digne, Descente de Shintani et restriction des scalaires, Journal of the London Mathematical Society. Second Series, vol. 59, no. 3, 867-880(1999).

[4] François Digne, Jean Michel, Representations of finite groups of Lie type, London Mathematical Society Student Texts, vol. 21, Cambridge University Press, Cambridge, 1991.

[5] Wee Teck Gan, Exceptional Howe correspondences over finite fields, Compositio Mathematica 118 (3), $323-344$ (1999).

[6] Paul Gérardin, Weil representations associated to finite fields, Journal of Algebra 46 (1), 54-101 (1977).

[7] Akihiko Gyoja, Liftings of irreducible characters of finite reductive groups, Osaka Journal of Mathematics 16 (1), (1979).

[8] Guy Henniart, Chun-Hui Wang, Weil representations over finite field and Shintani lift, Journal of Algebra 388, $311-323$ (2013).

[9] Noriaki Kawanaka, On the irreducible characters of the finite unitary groups, Journal of the Mathematical Society of Japan 29 (3), 425-450 (1977).

[10] Colette Mœglin, Marie-France Vignéras, Jean-Loup Waldspurger, Correspondances de Howe sur un corps p-adique, Lecture Notes in Mathematics 1291, Springer-Verlag, New York, 1987.

[11] Ilya I. Piatetski-Shapiro, Complex Representations of $G L(2, K)$ for finite fields $K$, Contemporary Mathematics, vol. 16 , American Mathematical Society, Providence, R.I., 1983.

[12] Ken-ichi Shinoda, The characters of Weil Representations associated to finite fields, Journal of Algebra 66 (1), 251-280 (1980).

[13] Takuro Shintani, Two remarks on the irreducible characters of finite general linear groups, Journal of the Mathematical Society of Japan 28 (2), 396-414 (1976).

[14] André Weil, Sur certains groupes d'opérateurs unitaires, Acta Mathematica 111, 143-211 (1964).

NCMis, Academy of Mathematics and Systems Science, Chinese Academy of Sciences, Beijing, 100190, P.R. China

E-mail address: cwang@amss . ac.cn 